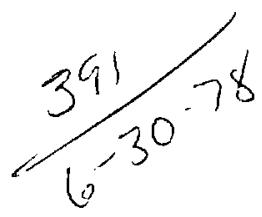

\title{
Der. 217
}

\section{MAGNETIC FUSION ENERGY QUARTERLY REPORT January through March 1978}

Scientific Editor: M. A. Harrison

General Editor: C. K. McGregor

April 22, 1978

Work performed under the auspıces of the UI $S$. Departrient of

Energy by the UCLLL under contract number W-7405-ENG-46.

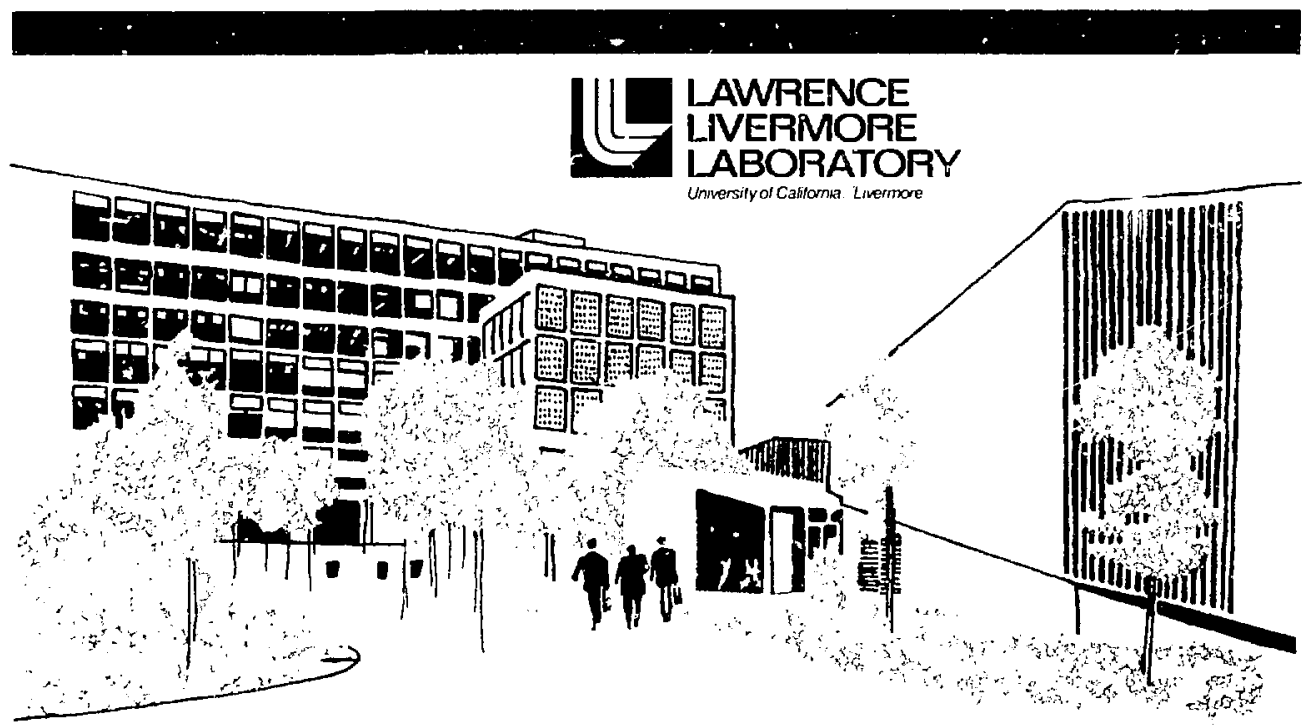




\section{แ4 \\ LAMRENCE LIVERMORE LABORATORY \\ iniversity of Cattomia/ Livermore,Calitomia/94550}

UCRL-50051-78-1

\section{MAGNETIC FUSION ENERGY QUARTERLY REPORT January through March 1978}

Scientific Editor: M. A. Harrison

General Editor: C. K. McGregor

MS. date: April 22, 1978

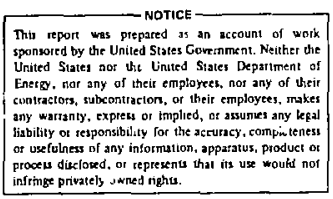




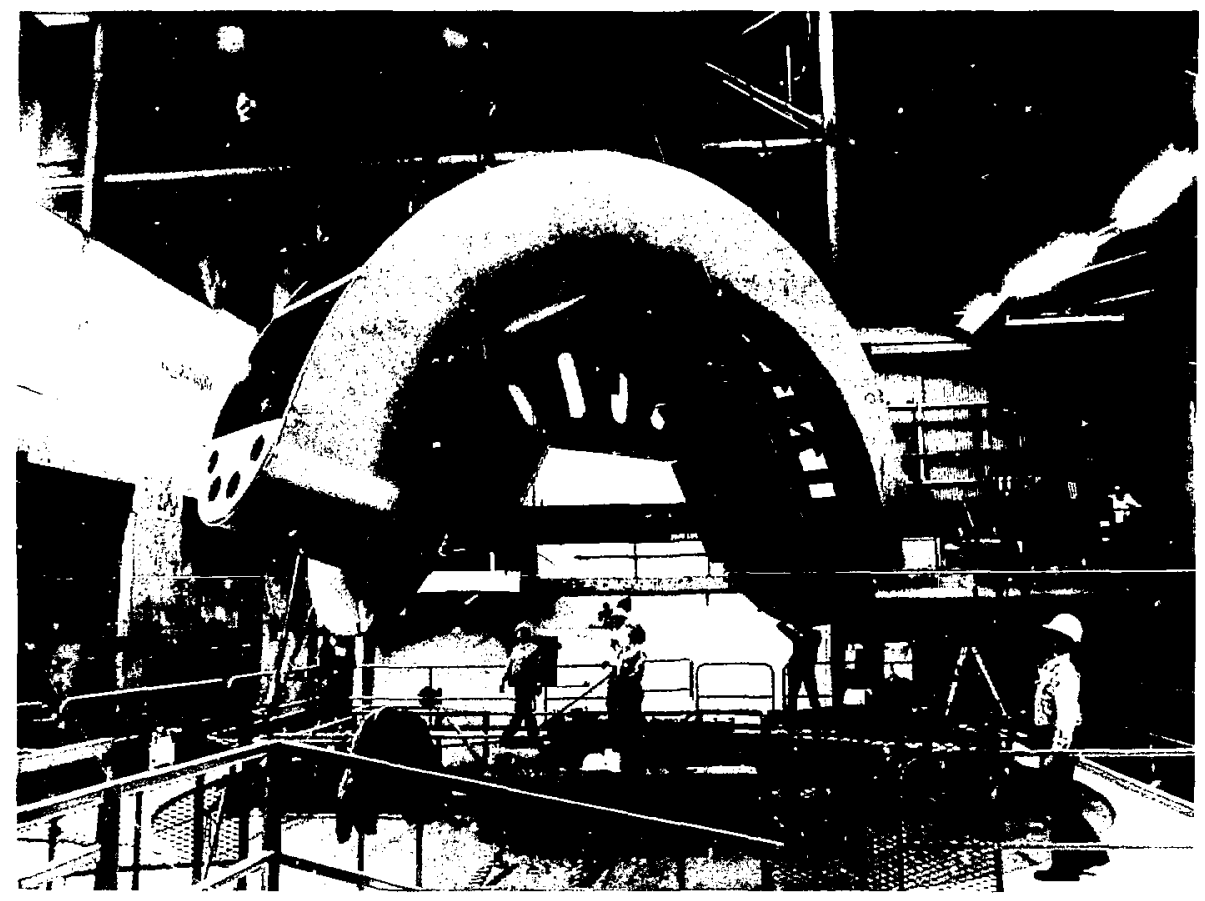

Frontinpiece. The Mirror Fusion Test Facility (MFTF) coil winder pictured in the last Magneric Fusion Encrgy Quarterly Reporr will be

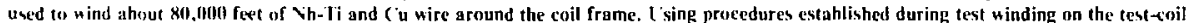
frams show n here, we can then hegin winding the twin coils that will create the magnetic field needed to confine the plasma in the $\$ 94$ million MFIt. 


\section{PREFACE}

Effective with the beginning of FY 1978, the Magnetic Fusion Energy Annual Report for the Magnetic Fusion Energy (MFE) Program at the Lawrence Livermore Laboratory (LLL) was replaced by quarterly reports; this is the second in the new series.

Our intent is to provide a timely summary of activities within the MFE Program at LLL. In a given Quarterly, not all MFE projects are necessarily represented. Throughout, details are kept to a minimum; readers desiring additional information are encouraged to read referenced documents or to contact the individuals engaged in the projects.

The information in each Quarterly is presented in the same sequence as in the Form 189 submissions prepared for the U.S. Department of Energy; the three main sections are Open Confinement Systems, Development and Technology, and Applied Plasma Physics. Each of these sections is introduced by an overall statement of the goals and purposes of the groups reporting in it. As appropriate within each section, statements of the goals of individual programs and projects are followed by articles containing summaries of significant recent activity and descriptive text.

The last Quarterly in each fiscal year will contain a list of all publications and presentations prepared by MFE personnel during that year. 


\section{CONTENTS}

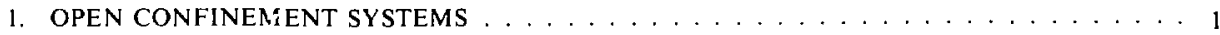

2XIIB

Large-Diameter Experiment

Introduction and Parameters Achieved $(2 \times 11 B$ team) $\ldots \ldots$

Radio-Frequency Probe (D. P. Grubb and T. C. Simonen $) \ldots \ldots \ldots \ldots \ldots$

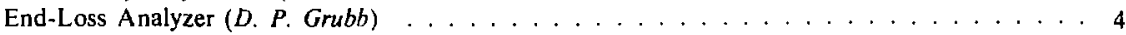

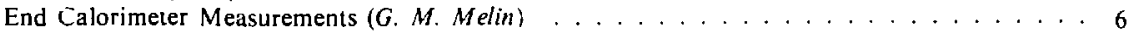

hatiai Fokker-Planck Calculations $(A, H$. Futch $) \ldots \ldots \ldots \ldots$

Preliminary Study of Cross-Field Plasma Injection

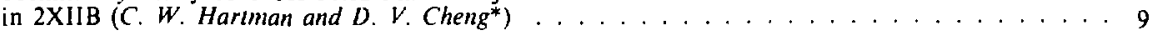

Low-Voitage Ion-Source Test $(J . E$. Osher $\ldots \ldots \ldots \ldots \ldots \ldots$

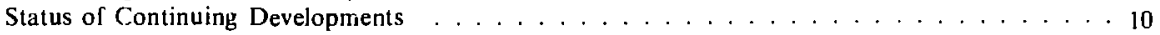

Electron Gun for the Stabilization of
the Drift-Cyclotron Loss-Cone Mode (B. G. Logan, J. D. Williams,

$D . P . G r u b b$, and G. G. Norlh $\ldots \ldots \ldots \ldots \ldots$

Measurements of Impurities in 2 XIIB $(R . P$. Drake $) \ldots \ldots \ldots \ldots$

HCN Laser Interferometer Diagnostic $(B, w$. Stallard $) \ldots \ldots \ldots \ldots \ldots \ldots \ldots$

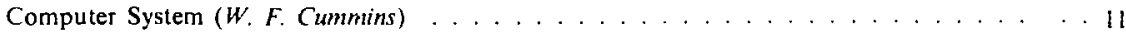

40-keV Ncutral-Beam Testing $(D . L$. Correll
and $R \quad H$. Munger)

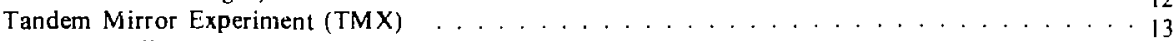

Magnet Alignment Requirements $(J . H$. Foote $) \ldots \ldots \ldots \ldots \ldots \ldots \ldots \ldots$

Construction (C. C. Damm, A. K. Chargin,

M. O. Calderon, L. J. Mooney. A. L. Hum.

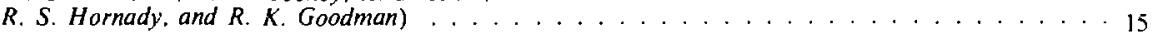

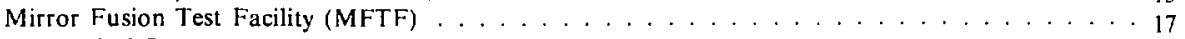

Technical Support . . . . . . . . . . . . . . . . . . . . . 17

Neutral-Beam Requirements (G. D. Porter. F. H. Coensgen,

R. S. Devoto. G. Leppelmeier, A. W. Molvik, J. E. Osher,

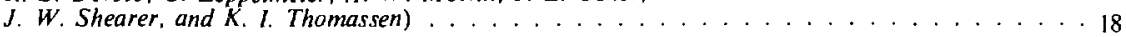

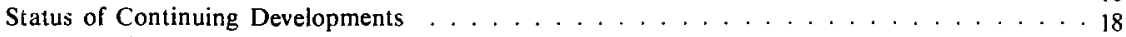

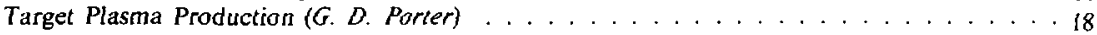

Ion Beam Dump Development $(R$. C. Ling and

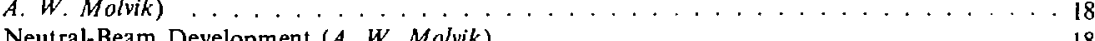

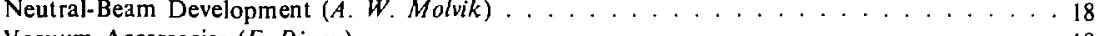

Vacuum Accessories $(F$. Dixnn $) \ldots \ldots \ldots \ldots \ldots \ldots \ldots \ldots$

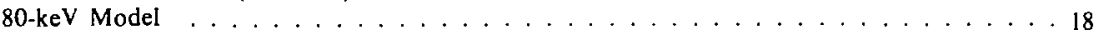

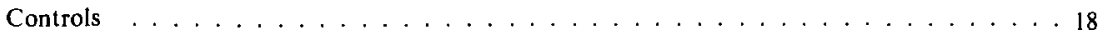

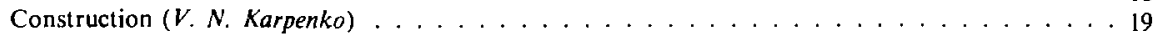

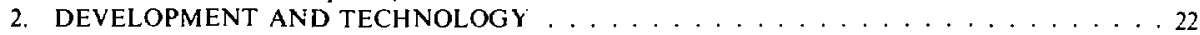

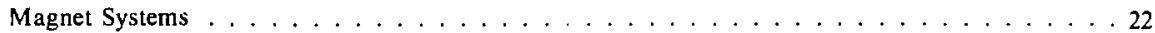

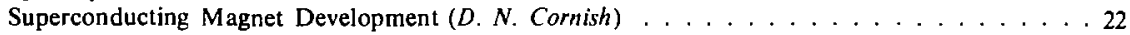

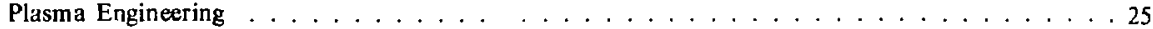

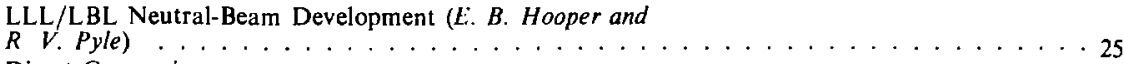

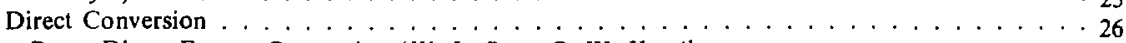

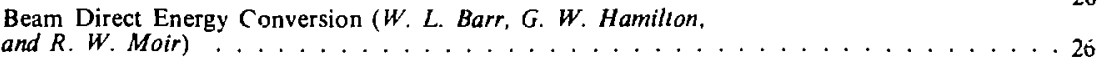

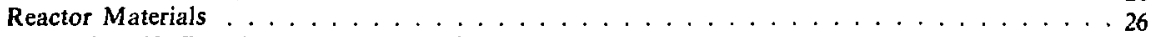

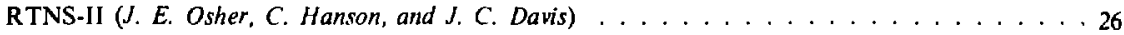

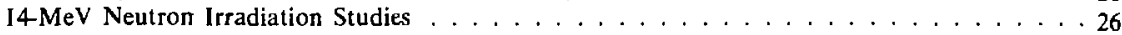

*University of Santa Clara. 
Radiation Damage in Superconductors $(R, M$. Scanlan $) \ldots \ldots \ldots \ldots$

Effect of Helium on Mechanical Properties of $\mathrm{Nb}$ and

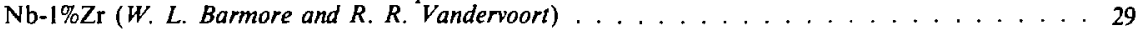

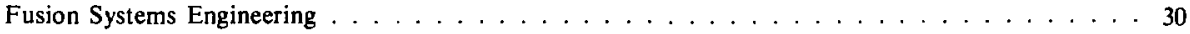

Reactor Design Studies . . . . . . . . . . . . . . . . . . . . 30

Performance Parameters for Fusion-Fission Power

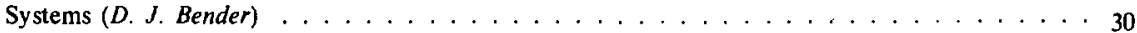

The Field-Reversed Mirror Reactor $(G$. A. Corlson $) \ldots \ldots \ldots \ldots \ldots \ldots$

The Moving-Ring Field-Reversed ivijrror Reactor Concept

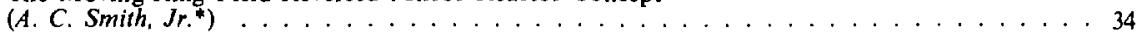

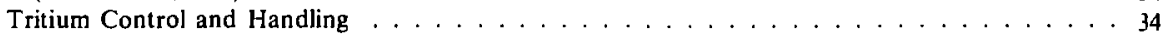

Tritium Processing and Control Using Active Metal

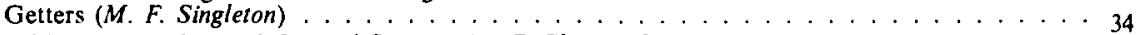

Tritium Processing and Control Systems $(A$. E. Sherwood $) \ldots \ldots \ldots \ldots \ldots$

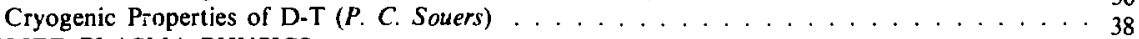

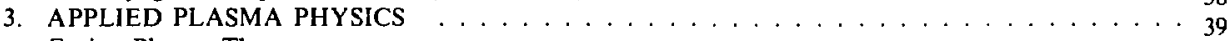

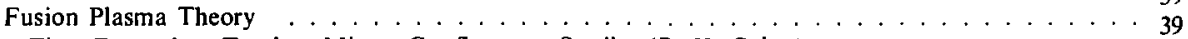

Tine-Dependent Tandem Mirror Confinement Studies (R. H. Cohen) . . . . . . . . 39

Superadiabatic lon Motion in the Presence of an Electrostatic

Wave in a Mirror Machine $(G$. R. Smith, J. A. Byers, and

L. L. Lodestro $\ldots \ldots \ldots \ldots$

Simple Rotating Field-Reversed Plasma Equilibria (B. McNamara) . . . . . . . . . . . . 39

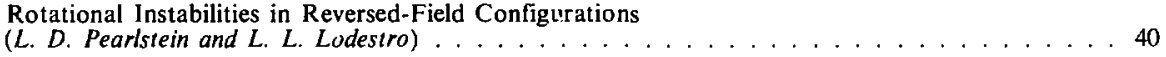

Orbital Resonances in Quadrupole-Stabilized Mirror Configurations

$(R$. H. Cohen, D. V. Anderson, and C. Sharp) . . . . . . . . . . . . 40

Spatial Eigenmodes for the Alfvén Ion-Cyclotron Instability

in Finite-Length Plasma $(D . C$. Watson and $T . D$. Rognlien) $\ldots \ldots \ldots \ldots \ldots$

Electron Effects in Ion-Current Field Reversal (D. E. Baldwin

and M. E. Rensink) . . . . . . . . . . . . . . . . . . . . . . 4l

Axial Profile of Eleciron Temperature via Monte Carlo Electrons

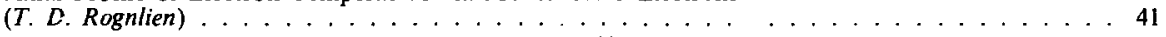

A Local Theory of Nonlinear Ion Dynamics in a Drift-Cyclotron

Mode (B. I. Cohen, N. Maron, and T. D. Rognlien) . . . . . . . . . . . . 43

3. Experimental Plasma Research . . . . . . . . . . . . . . . . . . . . 44

The Intense, Pulsed Ion-Neutral Source (IPINS) (D. S. Prono) . . . . . . . . . . . . . 44

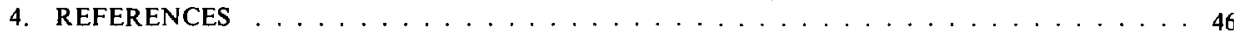

- LLL Participating Guest empioyed by the Pacific Gas and Electric Company. 


\section{MAGNETIC FUSION ENERGY QUARTERLY REPORT January through March 1978}

\section{OPEN CONFINEMENT SYSTEMS}

Lawrence Livermore Laboratory (LLL) has primary national responsibility for magnetic mirror programs, an approach pioneered here since the early 1950's. A goal of LLL's magnetic fusion energy (MFE) program is to provide the technology to develop a continuously operating fusion reactor. The heart of this reactor will be a plasma confined by magnetic mirror geometry and continuously sustained by injection of beams of energetic neutral atoms (such as deuterium).

There ar a three confinement systems now in operation or under construction at LLL: the 2XIIB, the Tandem Mirror Experiment (TMX), and the Mirror Fusion Test Facility (MFTF).

- 2XIIB relies on magnetic fields to confine a hot, dense plasma for a short time. It features $\mathrm{C}$-shaped magnetic coils that form the confining magnetic field. Their unique shape (in what is known as a yin-yang geometry) stabilizes the confined plasma by creating a magnetic field (a magnetic well) that increases in every direction from the plasma center.

- In 1976, we proposed a new idea: the tandem mirror concept. A tandem mirror reactor would contain a long solenoidal magnet terminated at both ends by conventional mirror cells. These cells would act as "end plugs" to prevent plasma leakage out the ends of the solenoid. The TMX is being constructed to test the principles of this concept.

- The MFTF now being constructed will bridge the physics and engineering gaps between present experiments and an experimental fusion reactor planned for operation by 1990 . The MFTF will use a superconducting magnet of yin-yang design (similar to the 2XIIB experiment). This magnet will be capable of continuous operation.

\section{XIIB}

The 2 XIIB magnetic-mirror machine was built to study the scaling of plasma confinement with ion energy. We have raised the mean plasma ion energy to $13 \mathrm{keV}$ and the peak energy confinement parameter $\hat{n} r_{\mathrm{e}}$ $\approx 10^{11} \mathrm{~cm}^{-3}$. $\mathrm{s}$. After reaching high betas $\left(\beta=8 \pi \mathrm{nW}_{\mathrm{i}} / \mathrm{B}_{\mathrm{vac}}^{2}\right)$, experiments attempting to achieve field reversal reached a field-reversal factor $\Delta \mathrm{B} / \mathrm{B}=0.9$. Experiments attempting to improve the microstability of mirrorconfined plasma, to increase plasma radius, and to further increase beta are underway.

\section{Large-Diameter Experiment}

The diameters of 2 XIIB plasmas have been doubled in a series of experiments begun this quarter. Improved electron energy confinement and an apparent reduction in anomalous ion losses were observed when the plasma radius was increased.

Introduction and Parameters Achieved. (2XIIB team)

The large-diameter plasma experiment initiated this quarter was the principal 2 XIIB experiment during the quarter. Larger-diameter plasmas are produced in 2 XIIB by aiming the neutral beams offaxis so that, when the beams have been ionized, the ion guiding center is displaced further from the axis, thus increasing the diameter of the plasma. This "paramagnetic" aiming is to the opposite side of the axis than that used in field-reversal experiments ("diamagnetic" aiming).

Figure 1 shows radial density profiles of beam attenuation, Thomson scattering, and charge exchange. These measurements indicate a mean plasma radius of $13.6 \mathrm{~cm}$. Since in these experiments beams were operated with hydrogen, this plasma has a size of seven gyroradii. Diamagnetic-loop measurements give further evidence that a large, hot-ion plasma has been produced.

That plasmas with larger radius can be produced means that stabilization via the streaming-plasma technique works fcr plasmas with radii larger than 2 to 3 gyroradii; this is not a trivial result, since it $m$ zans that finite-gyroradius effects were not responsible for the stabilization of the earlier, smaller-diameter plasmas. 


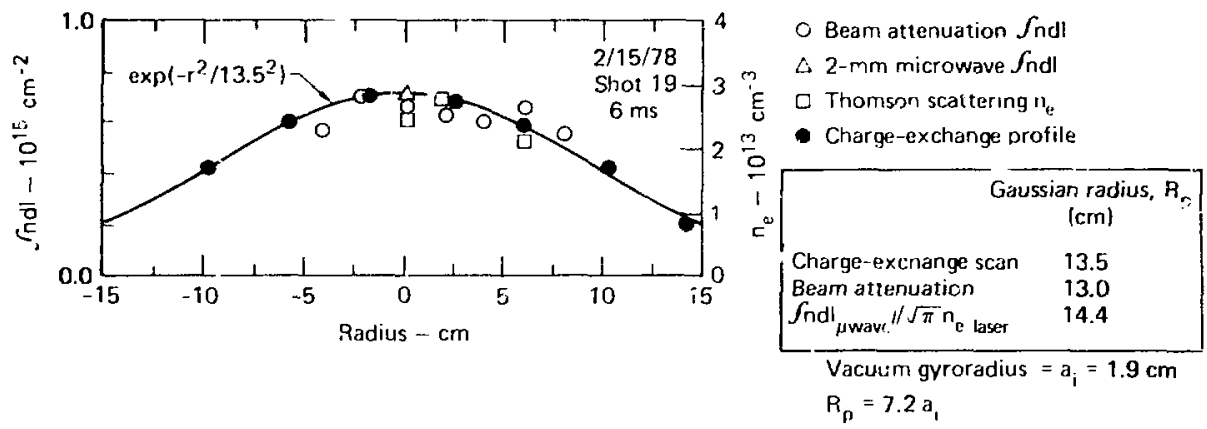

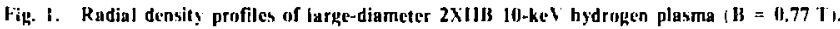

We have been conducting beam-aiming experiments over a wide range of conditions (see Ref. 1) and with different ion species in the neutral beams and streaming plasma. Table I summarizes data from our highest-beta shots. As can be seen (see also Fig 2), the plasma beta decisases with increasing plasma radius. This is expected without a dramatic increase in $n \tau$, since the beams are filling a much larger volume. The electron temperature decreases more slowly. A simple classical electron temperature model, where $T_{c}$ is electron temperature and $n$ is plasma density, gives a $T_{e} / n{ }^{4 / 5}$ theoretical scaling factor, whereas in earlier 2 XIIB operation, we found that $T_{\mathrm{c}} / \mathrm{n}$ was an experimental scaling factor that indicated the quality of electron confinement. In either case, we see that both $T_{e} / n_{e}^{4 / 5}$ and $T_{e} / n_{e}$ have improved with larger radius.

Figure 2 shows that the product $\hat{f} \tau_{E}$ of mean energy confinement time $\tau_{E}$ times central density $\hat{n}$ is independent of radius. ${ }^{*}$ Since $T_{e}$ is decreasing, it appears that the anomalous losses decrease with increasing plasma radius (note that fir $\tau_{E} /\left(2 \sqrt{2} n \tau_{d r a g}\right.$ ) increases with radius].

These experiments will be completed in April; we are obtaining considerable data and shall continue our analysis of it.

Radio-Frequency Probe. $(D . P$. Grubb and $T . C$. Simonen)

We have itseried a perpendicular, 13-tip if probe in $2 \mathrm{XIIB}$ in the east (vertical) fan $160 \mathrm{~cm}$ from the midplane. By inserting or withdrawing the probe,

*This comparison is not at constant beta. Further work will concentrate on com parisons of $\mathrm{r}^{+}$with radius for constant beta. we can measure the radial profile of the if fluctuations and map he recorded profile back to the midplane. For each data shot, not orly the envelope of the high-frequency oscillations Juring the entire shot is stored but also a $40-\mu \mathrm{s}$-long. high-speed transient record is stored. With the number of amplifiers and transient recorders available, we can study five spatial locations on each shot. Fast Fourier Iransform routines ${ }^{2}$ are used for frequency analysis of the data from the transient recorders.

The results of a ravial scan of the of fluciuations present in a large-diameter (large $R_{p} / a_{1}$ ) hydrogen plasma with a vacuum field strength of $0.77 \mathrm{~T}$ are summarized in Figs. 3 through 6 . The plasma density and electron temperature profiles are shown in

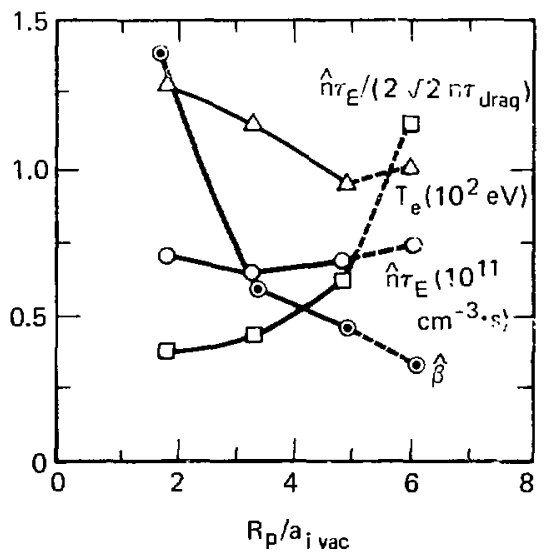

Fig. 2. 2 XIIB parameters is $\mathbf{R}_{\mathrm{p}} / \mathbf{a}_{\mathrm{i}}$. The data are for deuterium plasmas except for the right-most point. 
Table 1. Results of 2XIIB plasma-radius scaling experiments.

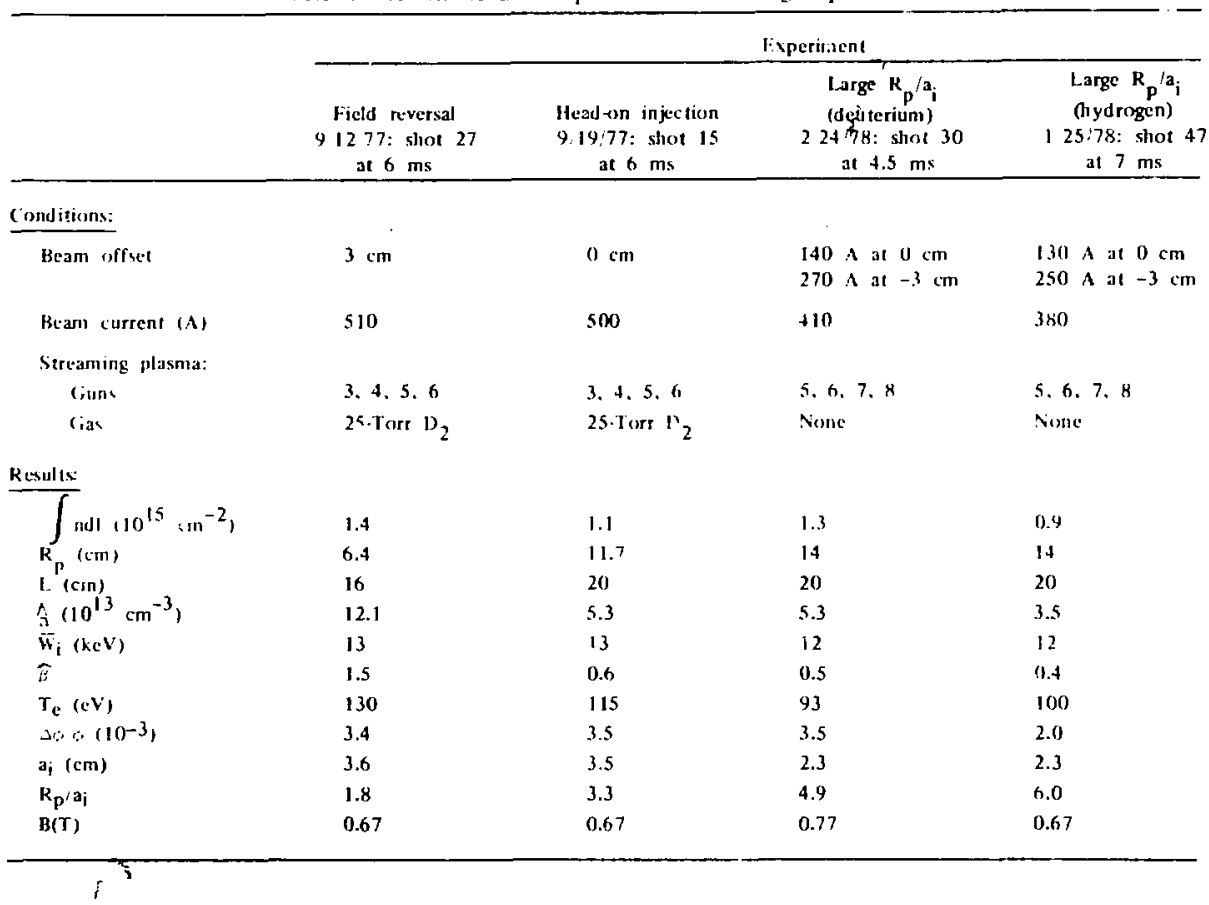

Fig. 3. The amplitude of the of fluctuations as recorded by the if envelope and the autocorrelated probe-1ip signal are shown in Figs. 4 and 5 respectively. These data are taken with a five-tip probe on several shots. The data points connected with lines are from one shot for both Figs. 4 and 5. Both figures show a reduced $\mathrm{rf}$ amplitude near the center of the plasma, though the effect is more pronounced in Fig. 5. This reduction may reflect the fact that the If oscillations recorded in Fig. 5 are only those at the cyclotron frequency. whereas the of envelope may be made up of signsls with many different frequencies.

The radial profile of lite frequency spectrum is shown in Fig. 6. Within shot-to-shot reproducibility, the mode exhibited a single, unique frequency $(10 \mathrm{MHz} \pm 0.1 \mathrm{MHz})$ over the entire diameter of the plasma. The observed frequency appears to be consistent with the $\beta$-corrected cyclotron frequency:

For the data reported above, the plasma was unstable to only a single mode. However, on several ocrasions during the large-diameter plasmá experi-

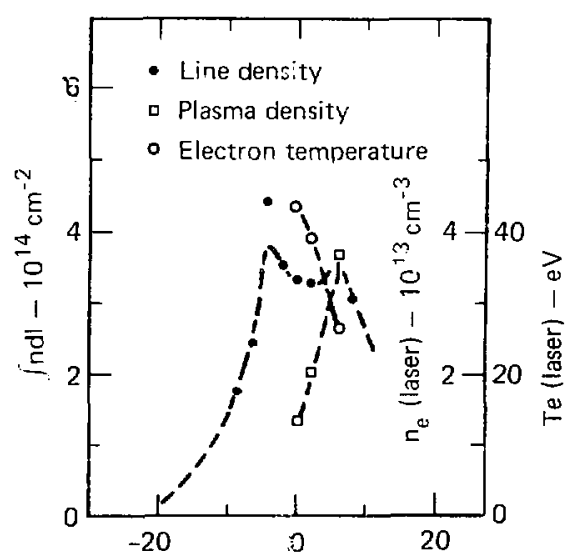

Distance frorii centerline at the midplane $-\mathrm{cm}$

Fig. 3. :adial profile of 2 XllB plasma in which of measurements were made. 


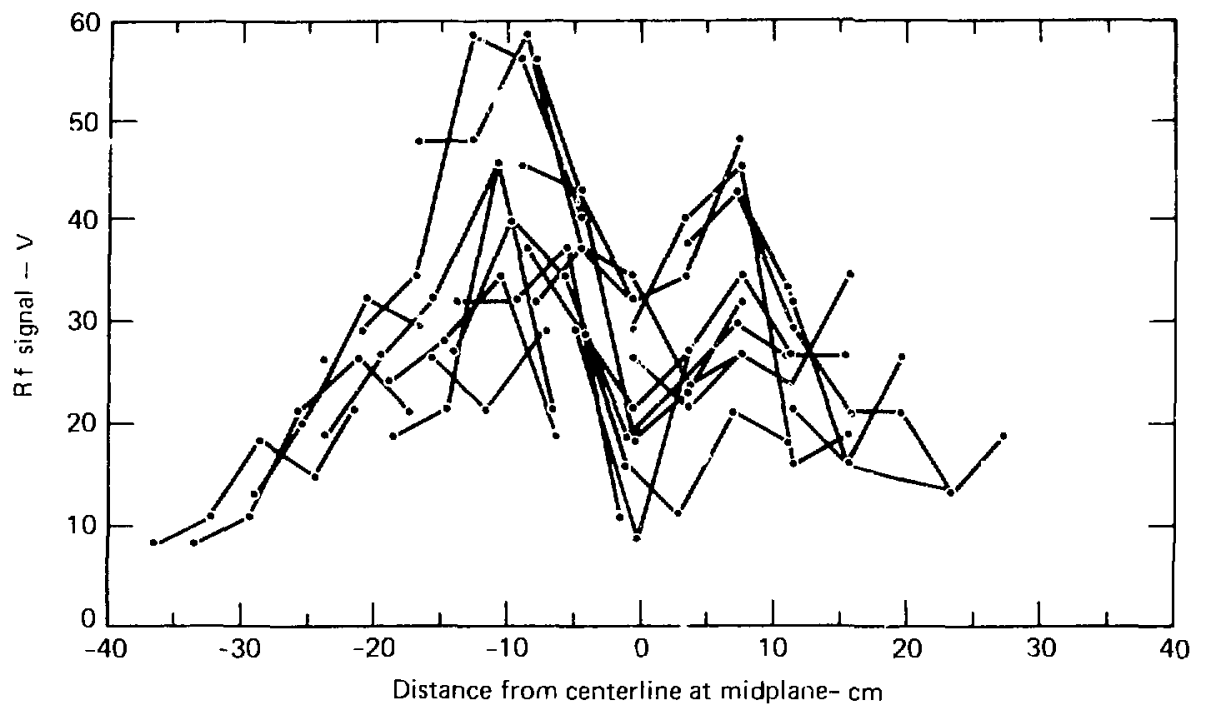

Fig. 4. Radial profile of average of ea elope signal from 4 io $6 \mathrm{~ms}$.

ments, we observed that the plasma was unstable to two modes with nearly the same frequency at the same time (Fig. 7). By yarying the neutral-beam current at a const?nt vacuum field strength, we measured the friculdency shift of these modes as a function of the central plasma beta $\beta(0)$. The frequency shift of both mudes shuw $n$ in Fig. 8 appears to be between a "long-thin" plasma approximation

$\mathrm{f} \approx \mathrm{f}_{\mathrm{vac}}[1-\beta(0)]^{1 / 2}$,

and a "bounce-averaged" model,

$\mathrm{f} \approx \mathrm{f}_{\mathrm{vac}}[1-\beta(0) / 2]^{1 / 2}$,

although the frequency of the low mode may not be the vacuum field value in the limit that $\beta(0) \rightarrow 0$.

That the if amplitude is localized on the edge of the plasma and that the plasma may be unstable to more than one mode at the same time are new observations that will receive more study during the next quarter.

\section{End-Loss Analyzer. (D. P. Grubb)}

The 2-in.-diam end-loss analyzer (ELA) developed by Molvik ${ }^{3}$ has been used to measure the flux of plasma particles impinging on the west-end wall of 2 XIIB during the large-diameter plasma ex- periments. In its present location, the ELA samples plasma from an ellipse that is approximately $5 \mathrm{~cm}$ high by $1 / 2 \mathrm{~cm}$ wide and centered at the midpl ne.

By varying the voltage of the ion repeller grid inside the ELA, we have measured the energy distribution of the plasma entering the ELA. The energy distribution of the plasma from the streaming-plasma gun is shown in Fig. 9(a): most of this plasma has an energy less than $250 \mathrm{eV}$. The energy disi ribution of the plasma reaching the EI A when the same streaming-plasma guns are fir:d in conjunction with the neutral-beam sources is shown in Fig. 9(b). Once again, there is a larg. suntribution to the end-loss current by particles with energies less than $250 \mathrm{eV}$, but there is now a significant current of ions vith energies greater than this amount. During the next quarter, we shall measure a third energy distribution: that of the plasma built up by the neutral beams and by only those st reaming-plasma guns on the east end of 2XIIB. By comparing these data with those in Fig. 9(b), we can measure the amount of streaming-plasma that is reflected by the potential created by the hot, trapped ions.

Under the same conditions, i.e., plasma buildup by the eas' streaming-plasma guns and by neutral beams, we can compare the current of ions measured by the ELA at energies less than $\sim 3 \mathrm{kT}_{\mathrm{c}}$ 


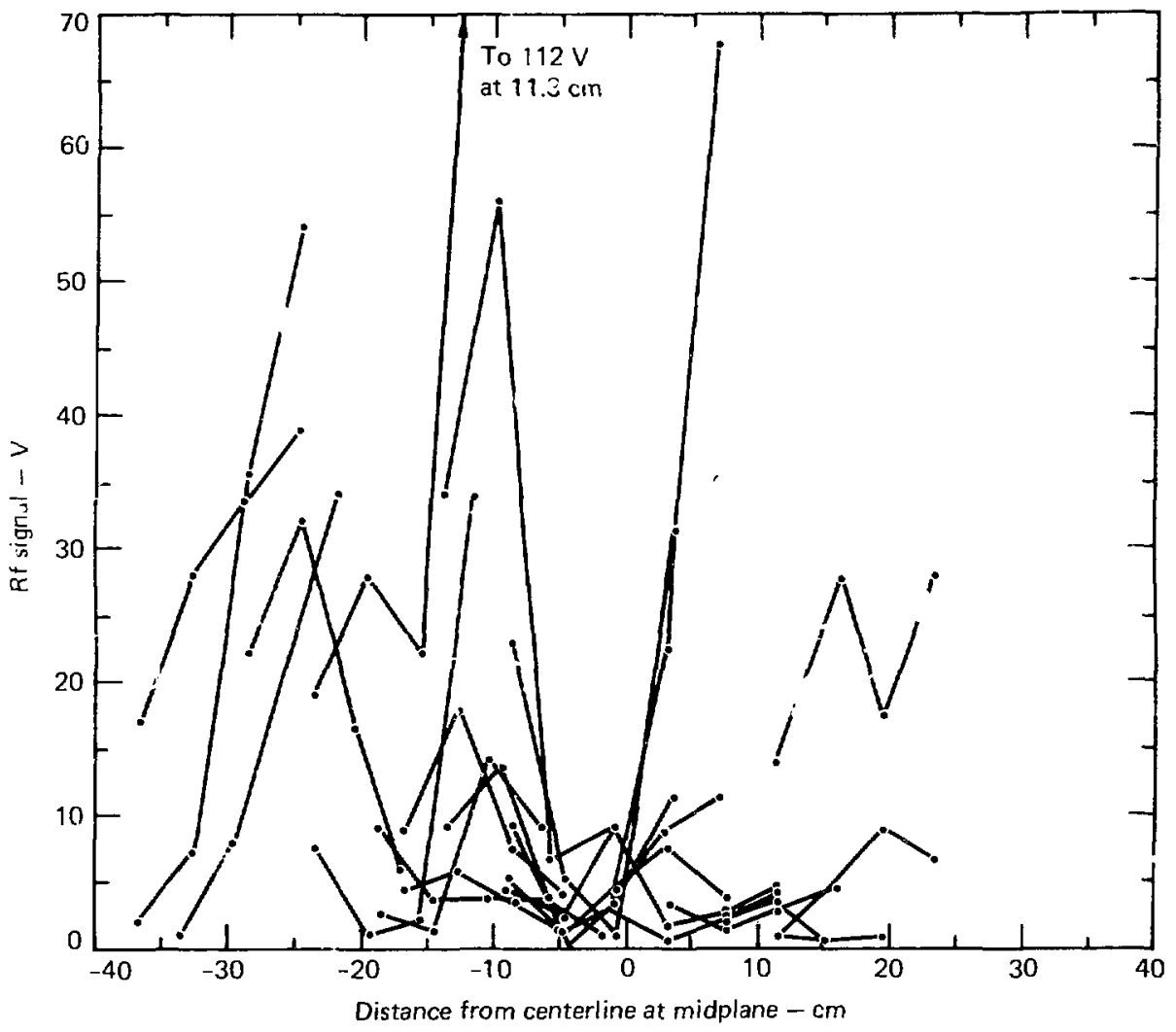

rïg. 5. Radial profile of $\omega_{d}$ averaged over $40 \mu \mathrm{s}$ (starting at $4 \mathrm{~ms}$ ).

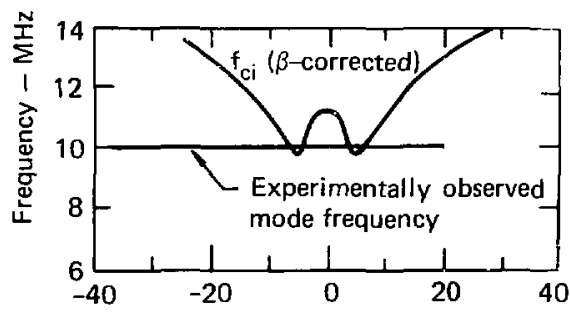

Radial distance from centerline at midplane $-\mathrm{cm}$

Fig. 6. Radial profile of observed frequency and the calculated $\beta$. corrected cyclutron frequency (for density distribution of Fig. 3 and $\mathrm{H}^{+}$plasms). 


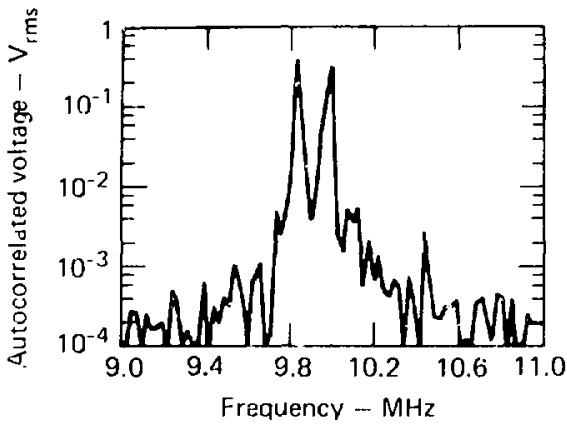

Iig. 7. Amplitude vs frequency for sutocorrelated prohc-tip signal.

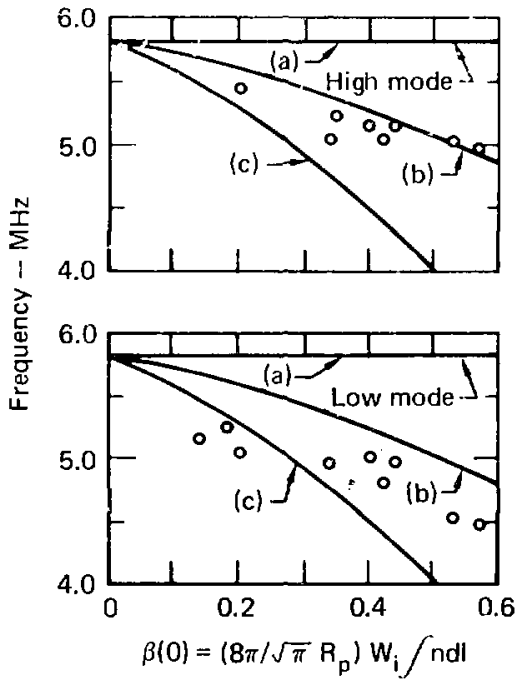

Fig. 8. Observed frequencies of two modes present at the same time as a function of the central plasma beta $\beta(0)\left(\mathrm{D}^{+}\right.$plasma ). For curves marked $(a), f=f_{, a c} ;(b), f=f_{\text {vac }}|1-\beta(0) / 2|^{1 / 2}$; and (c), $f=f_{\text {ver }}[t-\beta(0)]^{1 / 2}$.

with predictions of the minimum current required to maintain the DCLC mode at marginal stability. However, this requires that the energy distribution of the ion current to the EI_A be determined as plasma conditions change. To sweep out the energy distribution of the current several times during a single data shot, during the next quarter we shall
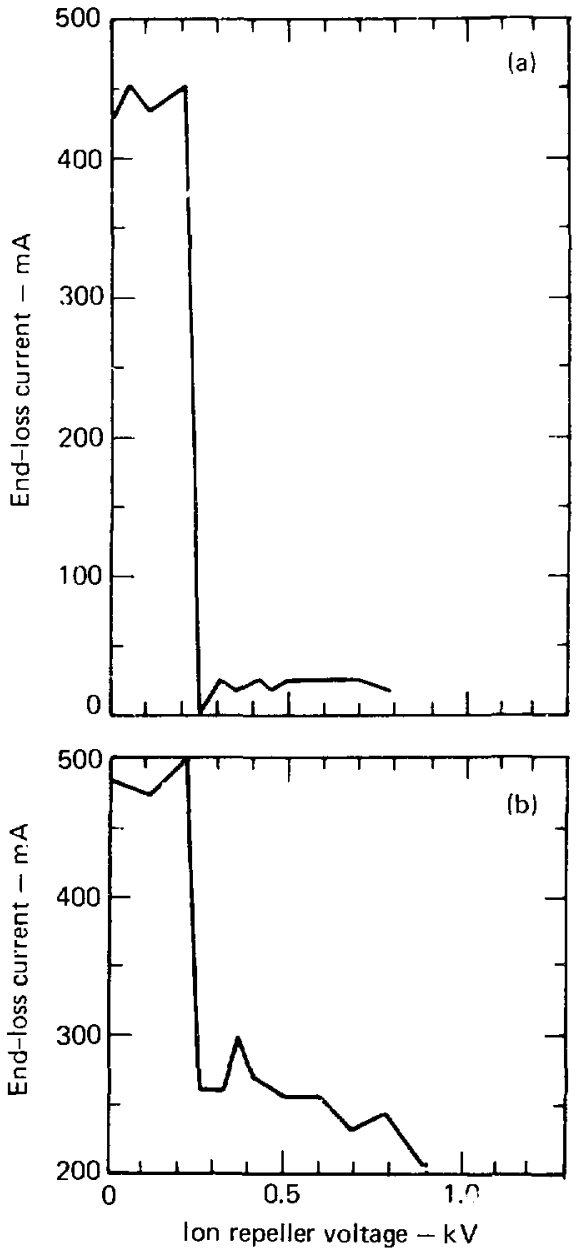

Fig. 9. Energy spectra of end-loss current: (a) streaming-plasma guns only, and (b) both streaming plasma guns and neutral-beam injection.

build a voltage-controlled power supply and ramping circuit capable of biasing the ion repeller grid from 0 to $1 \mathrm{kV}$ in less than $200 \mu \mathrm{s}$ and operate it with the ELA.

End Calorimeter Measurements. (G. M. Melin)

We have masured the end-loss energy leaking through tíe 2 XIIB fans during the large-diameter 
plasma experiments by using calorimeters located at each end of the machine (Fig. 10). Each calorimeter consists of an array of five copper plates $(2 \mathrm{~cm}$ $\times 6 \mathrm{~cm}$ ), the central plate heing divided into three smaller plates $(2 \mathrm{~cm} \times 2 \mathrm{~cm}$ The east caloritneter. when centered on the machine ceriter line. magnetically maps into a rectangular shape (about $30 \mathrm{~cm} \times 1 \mathrm{~cm})$, parailel to the $x-3 x$ is at the $z=0$ midplane (see Fig. 10), while the west calorimeter maps into an equivalent shape parallel to the $y$-aris. We can evalıate the total end-loss energy through a fan as follows

- The energy collected by a calorimeter, where the energ: profile is defined by the five plates, may be cunsidered as having been emitled by the associated rectangular area at the $2=0$ midplane.

- The end-loss energy is then calculated by assuming azimuthal symmetry.

In most experiments, the hot-piasma radius is stnaller than the rafial extent of the calorimeter mapping area $(\sim 15 \mathrm{~cm})$. The energy losses corr esponding to a radius larger than $15 \mathrm{~cm}$ arr then small and neglected in this end-loss energy evalu:ttion.

Experimentally, we find the follow:.tg:

- Most of the energy exits along or near the flux tubes of the stivis ing-rlasma guns.
- The energy profile is broader than that of the streaming-plasmat gun llux with no hot plasma present.

- Fyual energy depositions are obrerved on east and wesi calorimetcrs when symmetrically placed streaming-plasma guns are located on both ends of the machint.

- The energy received by the calorimeter durng a shot is consistent with other measurements in the end regions:

- flux of particles and electron temperiture from Langmuar probe datia.

-ion energy by a returding potential. gridded analyzer.

Figure 11 shows typical result oblained with a deutcrium plisma when the neutral-beatn carrent is varied: the total end-loss energy evialuated by calorimetry is plotted vs the total energy deposited by the beame during a shot. Only about $50 \%$ of the beam energy goes to the ends. The discrepancy raises several questions about the nature of other possible losser:

Losses via high-energy particles that go to the ends but miss the calorimeter because of their large Larmor radii; and

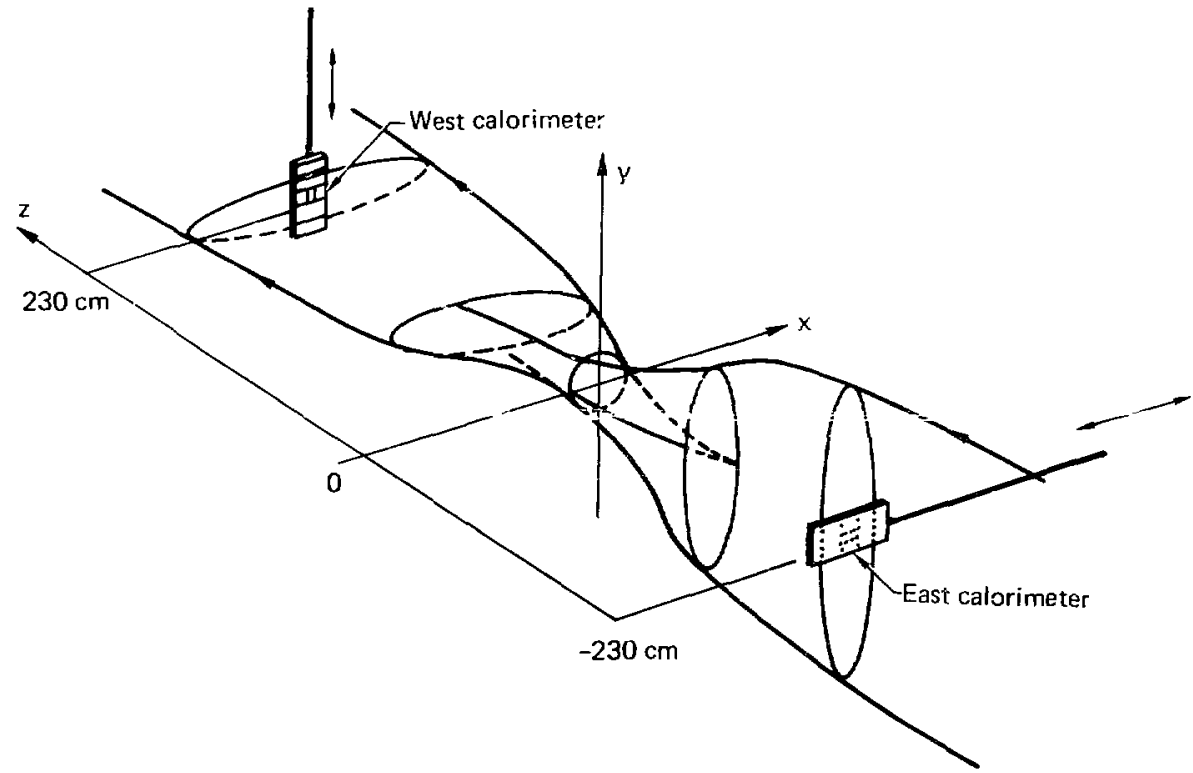

Fig. 10. Locations of end-loss calorimeters. 


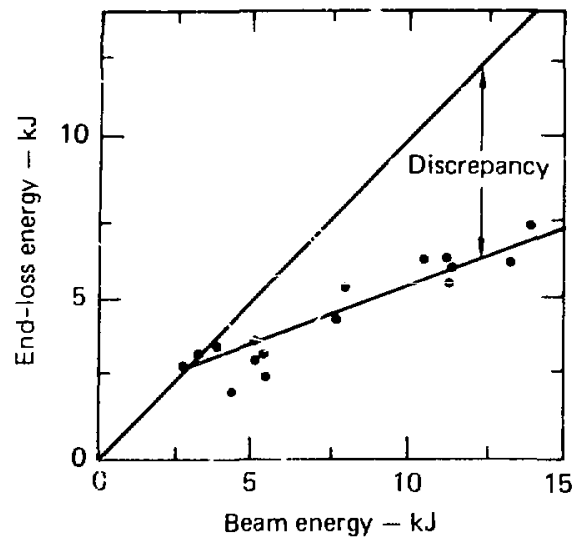

Hig. 11. Hind-lows tnergy (data points) vs deposited beam energy for various neutral-beam currents.

- Radiai losses at the midplane resulting from some rf activity, i.e., ej $x e r$ rf-enhanced radial diffusion or rf heating of ios s that are charge exchanging with coid gas at the plasma boundarv.

The classical Spitzer value for the average $n r$ product $\langle n T\rangle=4.4 \times 10^{7} \mathrm{~T}_{\mathrm{e}}^{3 / 2}(\mathrm{eV})$ agrees well with the experimental $\langle n r\rangle$ inferred from diagnostics measurements at low beam current: however, it is no longer relevant at high beam current. This behavior is similar to that found in the gas-box experiments reported elsewhere. ${ }^{4}$ We are investigating the role of of fluctuations and comparing experimental results with calculated results from a buildup code.

Radial Fokker-Planck Calculations. ( $A$. H. Futch)

We have modified the radial Fokker-Planck code (RFPC) to include quasi-linear diffusion. The Fokker-Planck coefficients were modified by adding additional terms calculated by integrating the quasi-linear coefficients derived by Rognlien for the HYBRID II code ${ }^{5}$ over a normal-mode distribution in angle. The HoIdren solution to the normal mode was used. ${ }^{6}$ A spatial diffusion term, which was added separately, was based on the crossfiel: diffusion coefficient of Kaiser. ${ }^{7}$

The quasi-linear terms caused a major change in most parameters calculated by the RFPC. For example, radial profiles were changed significantly. Also, previous runs in which the plasma decreased in radius as thermal gas eroded away the plasma surface before collapse occurred were stabilized by diffusion that moved the excess ions trapped in the plasma interior to the surface to compensate for the large charge-exchange losses.

Quasi-linear diffusion significantly reduced the electron temperatures, plasma putential, and particle lifetimes. Thesi calculations assumed ar $m=1$ mode and a perturbed potential consistent with experimental measurements. Calculations based on spatially constant Fokker Planck codes give electron temperatures of approximately une tenth the ion temperature. for $2 \times 1 i 3$ parameters, previous Fokker-Planck results would lead one to expect an electron tem $/$ erature $T_{e}$ of approximately $I \mathrm{keV}$; typical runs with the RFPC give temperatures of about $150 \mathrm{eV}$.

Since a number of physical effects not previously studied were included in the new results, it was desirable to remove these effects one by one from the code and then observe the charge in the calculated parameters. Figure 12 shows the effect on electron temperature at the center of the plasma when this is done. The results shown were obtained

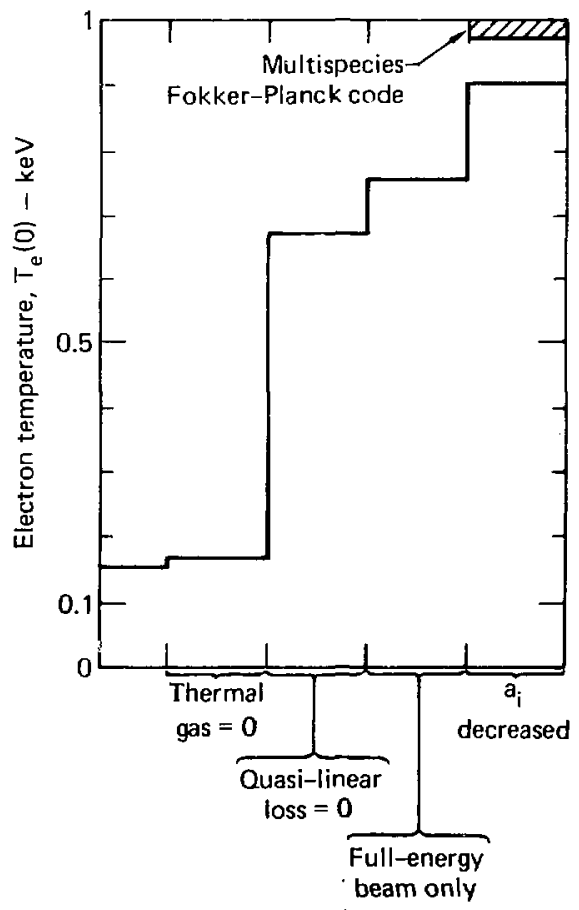

Fig. 12. Effect on colculated electron temperature at the center of the plasma of removal of various physical effects from the radial Folkker-Planck code. 
by readjusting the injected currents after each change until a new equilibrium was oblai: $d$ at the same density.

We found that charge exchange with thermal gas had negligible effec" in the ele. tron temperature at the plasma center $T_{e}(0)$. The greatest change in electrou temperature occurred when quasi-linear diffusion was removed from the code. The large change in $T_{c}$ results simply from an increase in the ion iifetime. and therefore the electron lifetime $\tau_{e}$. as turbulence is turned off. The electron temperature was found to be proportional to $\tau_{\mathrm{e}}^{2 / 5}$. A normal run consisted of three beams at full energy, half energy. and one-third energy. When the half- and thirdentrgy beams were turned off. $T_{c}$ increased by another $14 \%$. The last increase in $T_{e}$ resulted when the inagnetic field was incressed from 0.67 to $4.0 \mathrm{~T}$, thereby decreasing the gyroradius and increasing the center density. Thie center density increases because the ions trapped at the center contribute to the density over a smaller volume as the gyroradius decreases.

Figure 13 shows a comparison of the calculated electron temperature and density with experimental values obtained from a shot during the last quartur. The calculated electron density is in good agreement with measurements during the buildup and at equilibrium. but decays much slower than measured values after the beams are turned off. The calculated electron temperature is in reasonable agreement with the only measured point at equilibrium. The calculated electron temperature increases sharply after the beam is turned off because quasi-linear diffusion is set equal to zero to agree with the experimental observation that the instability stops immediately with beam turn-off. The faster experimental decay after beam turn-off indicates other cooling mechanisms such as cooling by the streaming plasma must be included to obtain agreement at low electron temperatures and fcllowing beam turnoff.

\section{Preliminary Study of Cross-Field \\ Plasma Injection in 2XIIB}

$$
\text { (C. W. Hartman and D. V. Cheng*) }
$$

Results of preliminary experiments in using crossfield plasma injection to achieve field reversal in 2XIIB are encouraging enough to merit further investigation.

These experiments in cross-field plasma injection were motivated by the possibility of achieving field reversal in 2 XIIB by transverse injection of plasma from a coaxial plasma gun. A recent reassessment of

\footnotetext{
-University of Santa Clara.
}

the requirements to obtain field reversal in 2 XIIR by neutral beams alone. taking into account electron effects, suggests that either the neutral-beam current or the confinement time must be increased by about a factor of 3 over present 2 XIIB values. Alternately, auxidary injection of approximately 6-kJ energy such as by transverse injection from a coaxial plasma gun might substantially reduce the required neutral-beam current.

The 2 XIIB experiment provides a high-quality environment in which to study cross-field injection usizg a coaxial plasma gun. Two important environmental aspects are the mininum-B magneticmirror field configuration and the high-vacuum. fast-pumpirg lechnology made possible by large. gettered surfaces iocated near the containment region. Furthermore, $2 \mathrm{Xll} \mathrm{B}$ his a complement of plasma diagnostics. Accelerated plasmas having 10 's of kilojoules of energy are available fron coaxial plasma guns, as compared with the $l-k J$ plasma energy that is present in a typical 2 XIIB experiment.

To assess the teasibility of cross-field injection. we began a preliminary series of experiments using a deflagration-mods, coaxial gun developed at the University of Santa Clara. These experiments were conducted for roughly 5 days during this quarter. Although the gun operated pocrly, only low-beta plasmas were trapped, and field reversal was not achieved, the results encourage us to continue these investigations. Figure 14 shows the measured line densities. The plasma trapped when both guns are fired simultaneously exceeds that trapped when the guns are fired individually.

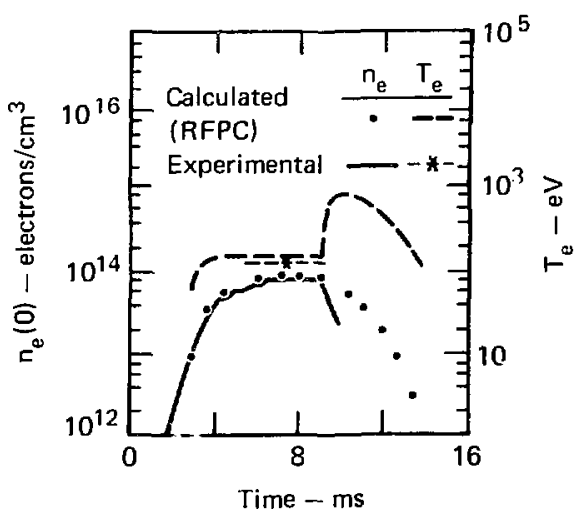

Fig. 13. Comparison between electron temperature and density values obtained experimentally and calculated with the radial Fokker-Planck code (RFPC). 


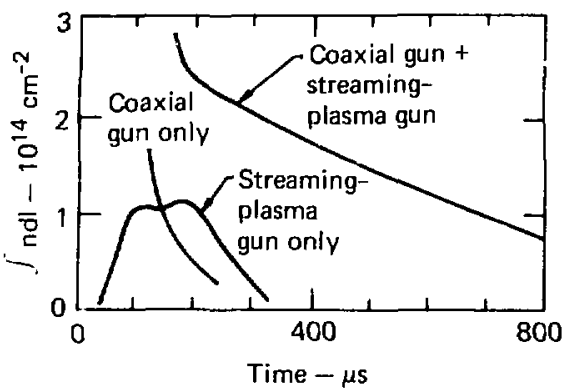

Fig. 14. I.ine densily $v_{3}$ time for stream and coaxial guns alone as well as stream and coaxial guns together.

\section{Low-Voltage lon-Source Test (J. E. Osher)}

High-voltage breakdown of a prototype streamingion source during early tests on the 2 XIIB has led to modifications and plans for additional tosting.

In January, we installed and began testing the prototype streaming-ion source. This source, which has a $10 \mathrm{~cm} \times 15 \mathrm{~cm}$ extraction area, was installed on-axis at the west end of the 2XIIB. An initial problem in which the 2 XIIB fringe magnetic field interfered with the operation of the electromagnetic gas valve of the source was corrected by encasing the valve within magnetic shielding and orienting the magnetic ax is of the valve so that it was perpendicular to the field of the 2XIIB end fan.

With this modification, we tested the source for operation in 2XIIB guide fields of $0.05 \mathrm{~T}$ to $0.3 \mathrm{~T}$, with up to 25-A peak extraction supply drains. When we fired $r$ nk 6 for the main 2 XIIB field, operation did not appear to be adversely effected. However, firing the $\mathrm{Ti}$ washer-stack streamingplasma guns for startup $y$ :nerally resulted in relatively prompt breakdown of tha high-voltage extraction system. Reducing startup $t$, the use of only a single streaming-plasma gun on the west end at best only delayed breakdown (irregularly) by 2 to $5 \mathrm{~ms}$.

We has considersd the possibility of such a breakdowil problem, and that was a basic reason for early if ng of the low-voltage source on 2XIIB; however, the problem was sufficiently severe to prevent useful operation with the 2 XIIB hot plasma. We had designed the three-electrode extraction geometry for the low-voltage ion source for output currents up to $200 \mathrm{~mA} / \mathrm{cm}^{2}$; however, because of a relatively large decel gap, we expected it to handle incident plasma at only up to $\sim 50 \mathrm{~mA} / \mathrm{cm}^{2}$ without breakdown. Thus, in early February we removed the source from $2 \times 11 B$ for examination and modiñcation.

Our examination of the fine, tungsten-mesh, ground-potential screen revealed that it had worked loose (because of heat cycling?) and ultimately been damaged (developed holes) fiom contact breakdown with the molybdenum wire grid of the decel. Also, the source face showed discoloration, presumably because the plasma from the streamingplasma guns had been concentrated into $\sim 2-\mathrm{cm}$ thick bands at the source location. This non uniform concentration of plasma probably also explains part of the unexpectedly severe high-voltage breakdown problem.

The ground screen has now been rebuilt of a heavier tungsten-mesh material and tightly stretched. It has a reduced decel gap that we expected can handle plasma currents of at least 120 $\mathrm{mA} / \mathrm{cm}^{2}$ beiore breakdown. Further tests are planned or. "XIIB. 'f breakdown problems still persist, use oil this type of source would probubly be restricted to regions of lower plasma density (perhaps the plasma boundary).

\section{Status of Continuing Developments}

Electron Gun for the Stabilization oi the DriftCyclotron Loss-Cone Mode. $B$. G. Lugan, J. D. Williams, D. P. Grubb, and G. G. North)

Because of the encouraging results of the electron-beam stabilization experiments at MIT $^{8}$ and on the basis of theoretical predictions by Baldwin aind Logan, we have developed an electron gun (Fig. 15) for stabilization of the drift-cyclotron loss-cone mode (DCLC) ir. the 2 XIIB experiment.

The gun is designed to provide $30 \mathrm{~A}$ of electron current at $20 \mathrm{kV}$ using a dispenser cathode that can be regenerated after exposure to air. ${ }^{9}$ The cathode (Fig. 15) is a tapered cone with a total emitting surface area of $6.6 \mathrm{~cm}^{2}$. In a uniform magnetic field, the election beam from this cathode is a $0.2-\mathrm{cm}$ thick annulus with a $2.5-\mathrm{cm}$ inner diameter. It is powered by a 1-ms-long pulse-forming network that is capable of supplying $30 \mathrm{~A}$ at $20 \mathrm{kV}$ into a matched load with a maximum default current of $60 \mathrm{~A}$. The body of the gun has been made long enough that the cathode assembly can be inserted inside the mirror throat if desired.

This electron gun has been built and testeci On a test stand, the gun has exceeded its design specifications, supplying $31 \mathrm{~A}$ at $23 \mathrm{kV}$ for $1.1 \mathrm{~ms}$. It is now ready for operation in 2XIIB.

Measurements of Impurities in 2XIIB. $/ R P$. Drake)

Extreme ultraviolet studies during this period, performed by the Johns Hopkins University, included further observation of known impurities and 


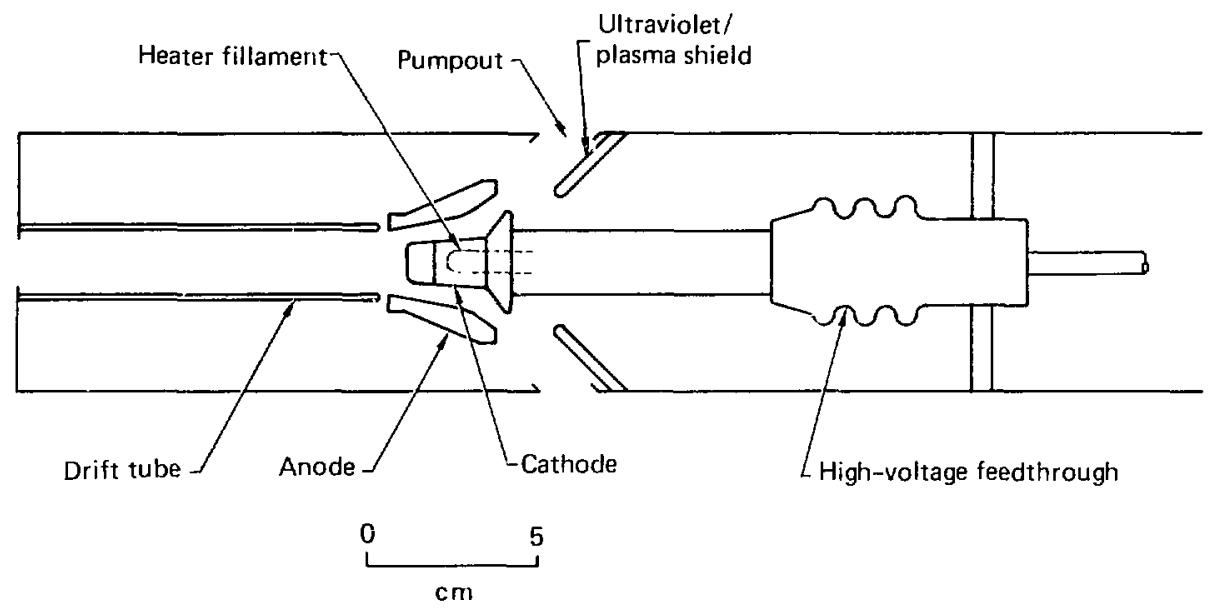

Fig. i5, The 2צJJB 20-kY, 30-A electron gun.

a sturiy of neon penetration from the end fans. To increise understanding of impurity distributions. densitues, power loss, and souıces, spatial and specii sl scans of oxygen, carbon, and titanium were performed. The plasma was stabilized with neon gas arc guns; neon emissiors were observed fiom the central plasma: and end losses were measured. As a result of recent work, we now believe that the neutral beams are the dominant source of oxygen in the 2XIIB, and that streamilišblosma impurities penetrate in small relative quantities and high ionization states.

HCN Laser Interferometer Diaznostic. $(B . W$. Stallard)

A new djagnostic being developed for ZXIIB is a combined Faraday rotation and interferometer diagnostic using $337-\mu \mathrm{m}$ radiation from an $\mathrm{HCN}$ laser. During this quarter, ve tested the interferometer arm with plasma.

The probing team path is shown in Fig. 16 . Measurements were made both with streamingplasma gun stabilization at both ends of the hot plasma and with stabilization only at the end opposite to the laser beam input. A comparison of line density measured with the $\mathrm{HCN}$ laser and with neutral-beam attenuation, measured perpendicular to the plasma axis, is shown in Fig. 17. The HCN laser data have been corrected for a longer beam path length by taking into account the plasma shape. As expected, the HCN line densities are somewhat greater than for beam attenuation since the probing beam passes through stabilizing plasma exterior to the hot plasma.

\section{Computer System. ( $V_{i}$. $f$. (immins)}

The principal addition to the $2 \times 11 B$ data acquisition and prusessing system during the last quarter was a computer system that wili serve as the executive and principal data processor for the TMX computer network. It can also be linked, if desired, to the future Beta II experiment.*

The primary elements of this system are a ceatral processing unit containing 128 thousand words o. memory, 14.7 million bytes of system disc memory, and 50 million bytes of disc data storage. For archival purposes, a nine-track magnetic-tape drive is included. To improve our graphics display capability, a "smart" terminal and a TV display interface were procured. An improved operating system was part of the package.

Our problem in linking the various computers in Building $\mathbf{4 3 5}$ stems from the fact that they are of differing vintages extending back 10 years. During March we adapted and configured $\boldsymbol{x}$ distributed system software package to our network requirements. This allows us-within a few days-to

*In FY 1979. we propose to bring 2XIlB back into operation after transferring some components of the present system to the TMX facility in FY 1978. Our objective is to provide an experimental facility to investigate single-cell physics questions related to MFTF, such as startup and stability. This experimental facility will be called Beta II. 


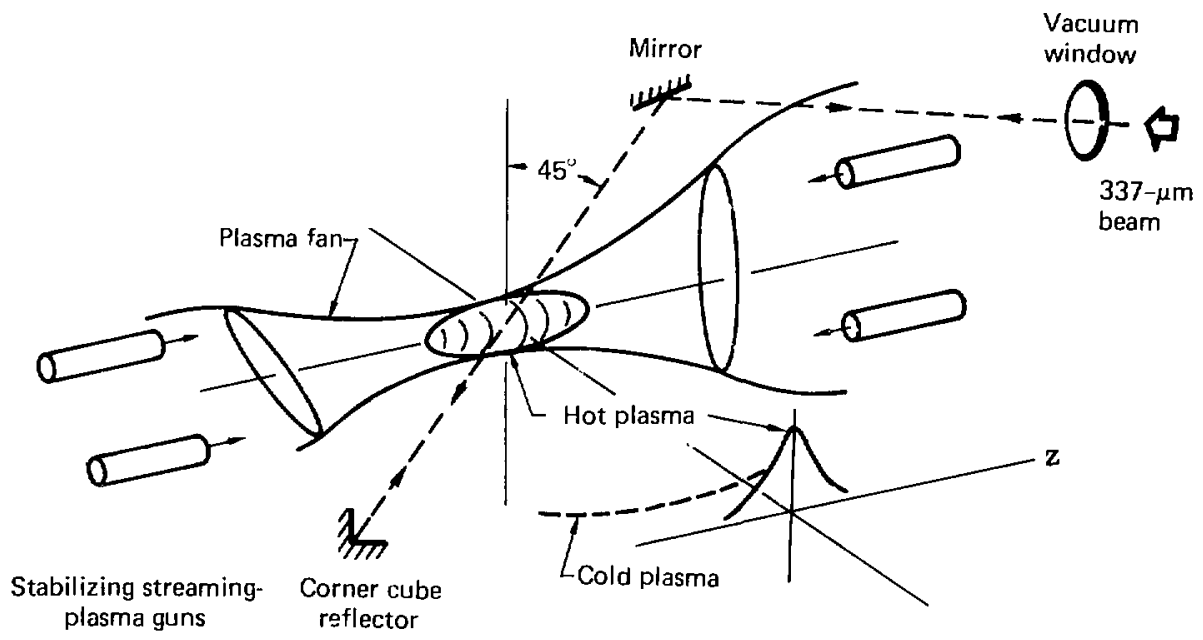

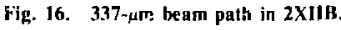

reconfigure the executive and satellite computers according to the demands of the experimental effort.

During the next quarter, our efforts will be directed toward defining and constructing a better management system for the data base than presently in use; the present search and retrieval procedures can be markedly improved. Applications programming stipport will continue for 2 XIIB experimental physics operations.

40-keV Neutral-Beam Testing. (D. L. Correlı and R. H. Munger)

To facilitate tests of the TMX battery-arc system, we have modified the $2 X$ test stand by the addition of a TMX-source gate valve on the side of the vacuum vessel. Late in January, the 2XIIB $10-\mathrm{ms}$ power supplies were discontinued and we began operation with the prototype TMX 25-ms supplies. Most of the remainder of the quarter was spent solving many complex electronic problems associated with the 2 XIIB 10 -ms power supplies and also the prototype $25-\mathrm{ms}$ supplies for TMX. The successful zero-offset $(Z-0)$ flat-grid set was used much of the time rather than the prototype TMX spherical grid, both to protect the latter from harm and to separate grid and electronic problems.

Because of the difficulty of localizing the electronic problems, we began a systematic investigation of the entire system. The operation of such

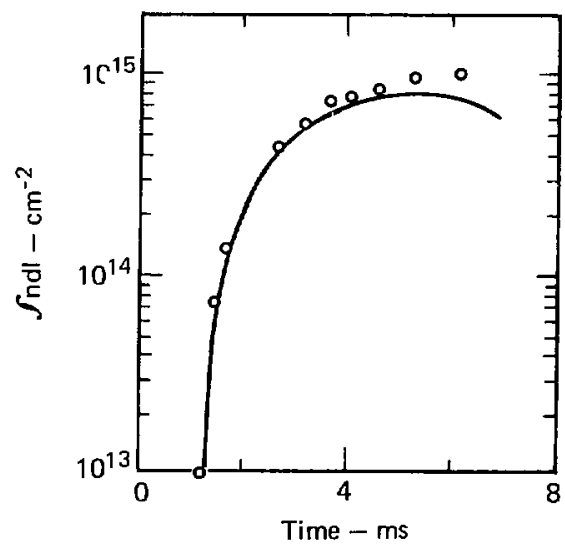

Fig. 17. Line density measured by the HCN interferometer (data points) and beam attenuation. Plasma stabilization is at both ends of the hot plasma.

components as the core stack (which protects the grid against damaging high-current surges during breakdowns) was examined in detail.

Many electronic problems were corrected in the timing chassis, in the arc charge, fire, and crowbar 


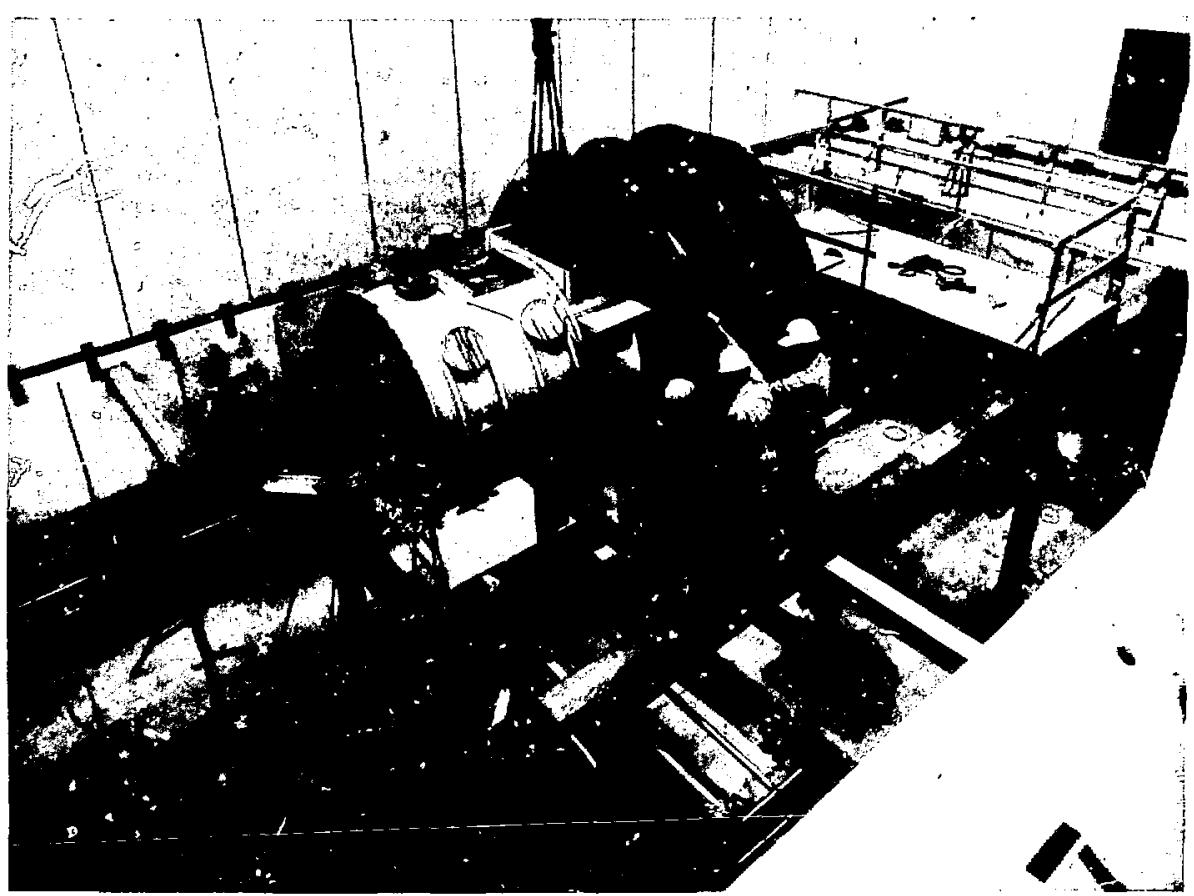

Fig. 18. Installation of the central vacuum chamber and solenoid magnets on the TMX support structure.

firing circuits, and in the accel fire and crowbar liring circuits. Most of these problems were cured by filtering and biasing circuits that were too sensitive to turn-on by noise.

The circuit that reduces the arc density during accel startup was iu und to contribute to the electronic problems. Consequently, startup without this circuit as proposed for the TMX battery-arc system was demonstrated when proper timing of the arc relative to the accel turnon was provided and the rate of rise of accel voliage was near that of the arc. This was made possible by the use of new control amplifiers.
When the power supplies operated more reliably, the $Z-0$ grid was run up to $37.6 \mathrm{kV}$ and $25-\mathrm{ms}$ pulse length: then the spherical grid was installed and operated. This grid ran up to $36.3 \mathrm{kV}$, at low accel current. with some difficulty; then later to $29 \mathrm{kV}$ with very good accel current. It was removed for exatnination because of voltage limitation, and the $Z-0$ grid was replaced. The $Z-0$ grid was then run up io $40 \mathrm{kV}$ with very good accel current.

A problem with the prototype spherical grid was found to be an internal breakdown batween the $\# 2$ grid lead-in wire and ground. The grid is being repaired; it will be installed and tested again next quarter.

\section{Tandem Mirror Experiment}

The Tandem Mirror Experiment (TMX) ${ }^{4,10}$ will test a new principle for improved piasma confinement in mirror systems. The basic idea is to reduce the plasma loss rate by electrostatically plugging the ends of a solenoidal central confinement region using the high positive ambipolar potential generated in minimum-B end plugs. Each end plug will be driven by the injection of rieutral beams from 12 sosirce modules, in a manner similar to that used in the 2XIIB experiment. 
The TMX has three fundamental objectives:

- To demonstrate the establishment and maintenance of a potential well between two mirror plasmas;

- To develop a scalable magnetic geometry, while keeping macroscopic stability at high beta; and

- To investigate the microstability of the plug-solenoid combination in order to maximize the plugdensity/injection-power ratio.

Possible secondary objectives that have important reactor implications include the study of enhancer, radial transport $i_{i i}$ the solenoidal cell and the accumulation of thermalized alpha particles in the central plasma.

The key physics parameters projected for the TMX experiment are listed in Table 2.

Ti ble 2. Key physics parameters for the TMX experiment.

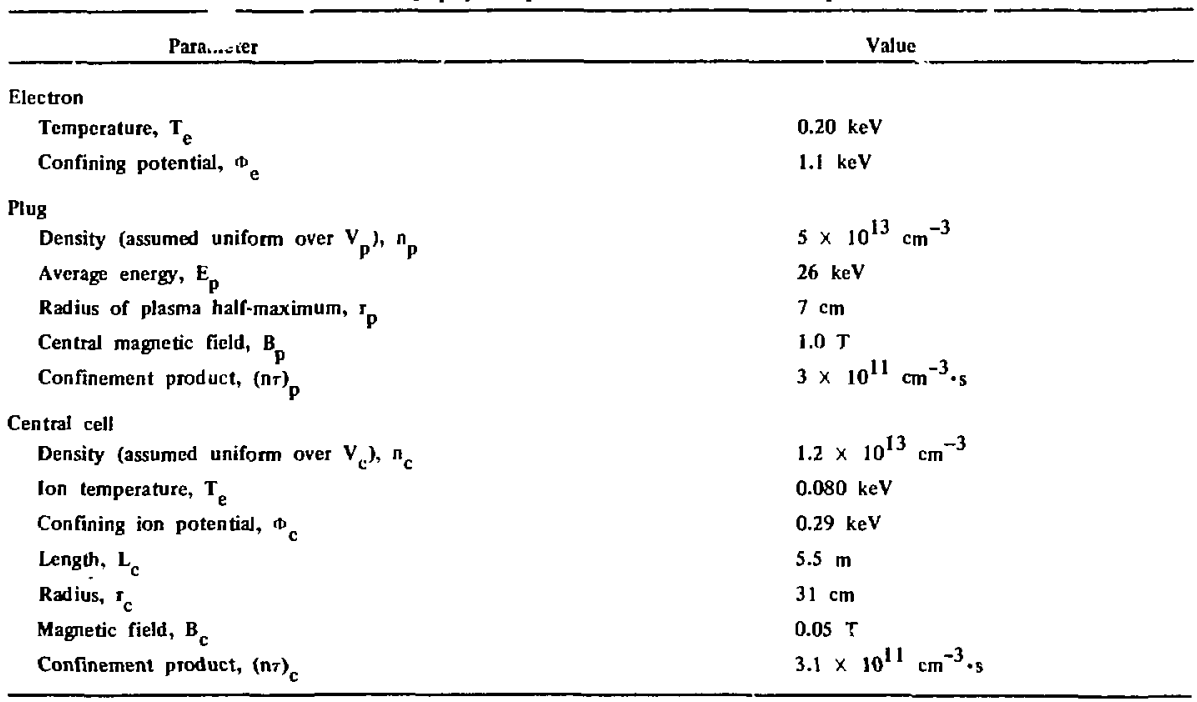

\section{Magnet Aligmment Requirements}

(J. H. Foote)

The accuracy necessary when installing the TMX magnet coils has been determined from magnetic-field and particle-trajectory calculations.

We have completed a seriec of drift-surface and magnetic-field calculations in which we estimated the accuracy with which the elements of the TMX magnet set must be magnetically aligned, " for a 0.05 -T field at the center of the solenoid. 1

One might think that misalignment of the magnet elements could distort the curvature of the magnetic-field lines in thu solenoidal section enough so that radial drifts of the trapped ions, and even radial losses, could occur. Radial shifts indeed take place; however, our calculations show that the drift surfaces of ions magnetically trapped in the solenoidal section provide an insensi'ive indicator of misalignment problems. The intersections of these drift surfaces with the solenoidal mioplane tend to remain closed and to shift with respect to the solenoidal axis by an amount comparable to or less than the misalignment shifts of the plugs and transition C-coils.

The more sensitive measure of the required alignment accuracy is either the position of a drift surface at a plug midplane calculated for ions that pass through the solenoid and reflect at the outer mirror 
regions of the plugs or, similarly, the mapping along magnetic-field lines of the plasma cross section at the solenoidal midplane to a plug midplane. The solenoidal plasma should map to the region of the plug plasma so that ions escaping from the solenoid can be reflected by the plasma potential of the plug. If we use as the criterion that a $31 \cdot \sim \mathrm{m}$-radius circle at the solenoidal midplane (repr intirg the plasma there) should map into an $a^{r}$. oximate circle (with about a $7-\mathrm{cm}$ radjus) at. pllng midplane with the center shift: magnetıc axis of the plug, then a plug set (baseball plus nested C-coils) and the corresponding pair of transition $C$-coils must be aligned with respect to one another to within about $0.5 \mathrm{~cm}$. The combination of the plug set and transition C-coils, when well aligred with respect to one another, can be misaligned with respect to the solenoidal axis by $P$ considerably larger amount and still satisfy our criterion.

An additional ohematinn made during the series of drift-surface calculations summarized here is that we must guard ayjaiost slight dips in $|B|$ in the neas!y uniform sole:toidal magnetic field region. Such magnetic-field fluctuations can temporarily trap ions with pitch angles near $90^{\circ}$, which in turn can cause the particles to drift radially and be lost. A smoothing of the solenoidal magnetic field ciminates this problem.
Construction

1C. C. Damm, A. K. Chargin. M. O. Calderon,

L. J. Mooney, A. L. Hunt, R. S. Hornady, and

R. K. Goodman)

At the end of this quarter, the TMX construction project was about $65 \%$ complete, with debug operation of the system scheduled for October 1, 1978.

Ain engineering description of TM $\mathrm{X}$ by subsystem has been given in Ref. 10; the status of each subsystem is summarized here.

Magnet System. All coils for the magnet system are either wound or in the process of being wound; tre status of the coil fabrication is shown in Table 3.

A finite-element stres 3 analysis of the restrainin? structure of the plug magnet assembly verifies that the design is conservative. The structure is now be1.ig fabi rated.

The solenoid magnets have been installed, Fig. 18; instaliation of the other coils, which is keved to th ...mint. rguence of the vacuum tanks, will start in May.

Vacuum System. Tl.e central-cell tank has been installed on the support structure, Fig. 18. The fabrication of the plug tanks is underway; we expect delivery of the first sections in mid-May. The liquidnitrogen-cooled (LN-cooled) panels are leing fabricated, and delivery should meet the assembly scheduled to begin in May. Fabrication of the

Table 3. Status of TMX magnet construction.

\begin{tabular}{|c|c|c|c|c|c|c|}
\hline Magnet & $\begin{array}{l}\text { Winding } \\
\text { fixtures }\end{array}$ & $\begin{array}{c}\text { Potting } \\
\text { case }\end{array}$ & Winding & Potting & $\begin{array}{c}\text { Vacuum } \\
\text { case }\end{array}$ & $\begin{array}{l}\text { Cap and } \\
\text { leak cileck }\end{array}$ \\
\hline C-coil No. 1 & done & done & done & done & done & done \\
\hline C-coil No. 2 & done & done & done & done & done & done \\
\hline C-coil No. 3 & done & done & done & done & done & done \\
\hline C-coil No. 4 & done & Lione & don: & done & in progress & in progress \\
\hline $86^{\circ}$ tranc. No. 1 & done & done & di;ne & done & $\begin{array}{l}\text { parts due } \\
\text { March } 3 t\end{array}$ & \\
\hline $86^{\circ}$ trans. No. 2 & NA & done & do.se & done & $\begin{array}{l}\text { parts due } \\
\text { March } 31\end{array}$ & \\
\hline $180^{\circ}$ tans. No. 1 & available & done & done & & $\begin{array}{l}\text { parts due } \\
\text { April ! }\end{array}$ & \\
\hline $180^{\circ}$ trans. No. 2 & NA & done & in progress & & $\begin{array}{l}\text { parts due } \\
\text { Apr: } 8\end{array}$ & \\
\hline Baseball No. 1 & done & done & done & Jone & done & \\
\hline Baseball No. 2 & done & done & in progress & & available & \\
\hline Octupole No. 1 & done & done & done & & $\begin{array}{l}\text { parts dne } \\
\text { April } 21\end{array}$ & \\
\hline Octupole No. 2 & NA & done & in progress & & $\begin{array}{l}\text { parts due } \\
\text { April } 21\end{array}$ & \\
\hline
\end{tabular}


vacuum feedthroughs for these panels is also in process.

The titanium getter: have been designed and are being procurcd.

Injector System. The 40-keV source modules consist of an arc chamber and an extractor assembly. Components for the arc chamber are in hand and assembly has started. Parts for the extractors are due from the vendor early in May. The fixtures for assembling and servicing the injector magnetic: shieid and neutralizer assembly have been completed and are in use.

Mounting fixtures for the streaming-plasma guns are being fabricated and a gas-box design is complete. The prototype piezoelectric valve for the gas box has been successfully tested: the valve opens in $<1 \mathrm{~ms}$. with a gas flow rate of $\sim 47$ Torr-litre $\cdot \mathrm{s}^{-1}$.

We have received for lesting two more accelerator-modulator power supply units. The design of the battery power supply for the filaments and protolype testing is complete and production is procceding. The battery power supply for the arc is in a final testing stage, with minor rework of the controls underway.

Facilities. The support structure for the vacuum tanks has heen installed in the TMX pit, Fig. 18. All power-supply tiers have been completed, as has a complete grcunding-plane system.

The installation of the control racks and the raised floor is complete and the racks are being wired. The electrical installation contract for highvoltage power and other general utilities is in the procurement cycle, with work expected to begin in May. Power for general assembly requirements in the TMX pit has been installed.

Design work is proceeding with the LLL LN supplier for the installation of tws i 1,000-gal tanks to replace an existing 7,500-gal tank that has insufficient capacity for the TMX requirements.

Cortrols. Design of the magnet control system is complete, fabrication of components has begun, and software is being developed. The design and fabrication of the safety monitor chassis and master timing distribution amplifier have been contracted and are underway.

The neutral-beam control chassis, including controls for battery filament, battery arc, and accel/decel power, is being designed. The telemetry unit design is complete, with prototype testing dependent on delivery of parts.

\section{Diagnostics.}

Thomson Scattering System. The four-pulse ruby laser is on hand; only one electronic problem remains to be corrected before the laser ir accepted from the vendor. We have decided to locate the laser power supplies on the shelf at the east end of the pit. The basic optical design of the Thomson scattering system is well along, and the 66 individual componen*s. ?re now being s.r.cified and ordered. Mechanical designs of the subsystems that hold and enclose these optics are progressing ciose to schedule. The design and specification of associated electronic components and interfacing has begun. A suitable photomultiplier tube has also been sel-sted. and a set of these tubes will be ordered sum...

Healy-ion Probe. During this period, a contract was signed with Rensselaer Polytechnic Institute (RPI) to assume technical responsibility for the implementation of a heavy-ion beam-probe system to measure the radial potential profile in the TMX midplane. Under this contract, RPI will provide information and design to L.LL for the construction of the necessary components and supply personnel at LLL to activate and operate the system when TMX begins operation.

A $\mathrm{n}$ ion suurce based upon RPI designs and approved by LLL was constructed and is being tested on RPI's test stand. Calcslations by LLL and RPI have identified suitable locations for the effective source point and the secondary-ion det ctor. Detection grids using these locations have been calculated by both groups for the vacuum field so as to establish a base line against which system operation may be compared.

Once we knew the source and detector location. we began designing the necessary vacuum chambers that will contain various beam apparatus and be mounted on the TMX center-cell tank.

Aiso, a control system was conceptually designed in sufficient detail to allow purchase of the majority of the necessary commercial componen processor and some supporting modules have been sent to RPI for systems developintent. The design of special necessary interface boards has also begun.

For the axial beam system, we acquired an ion source and recovered suitable vacuum tanks and pumps from surplus equipment. Because the rontrol system will be identical to that for the transverse system, its design is progressing. Most of the necessary power supplies are also available from LI.L surplus. We expect to test the axial beam source during the next reporting period.

Low-energy lon Analyzer. This diagnostic provides the energy spectrum of the electrostatically corfined ion distribution in the centrai region between the plugs.

We reviewed the originally proposed experimental method ${ }^{10}$ (the time-of-flight method) and retained $i$ with some modifications dictaied by calibration of the low-energy neutral-deuterium atom detector. The neutral beam incident on the plasma has been increased from $1-\mathrm{keV}$ to $20-\mathrm{keV}$ to 
take advantage of the previous devetopment and current availability of the LBL 50-A source. The resulting charge-exchanged neutral flux from the plasma will be interrupted by a chopper wheel rotating at $24,000 \mathrm{rpm}$, with a slit width corresponding .o a $\mathrm{I}-\mu \mathrm{s}$ transmission tim. ' $\mathrm{d}$ a $2-\mathrm{m}$ flight path, as described in Ref. 10. Thic neutraldeuterium atom detector at the end 0 , the flight path has been changed to a microchannel plate with an electrically grounded front face. This w: it is commercially available as a component of an imaging electron multiplier. The potential advantage of this detector is a significant reduction in noise. an im. portant attribute at the arcicipated counting rates. All detectors must bu calibrated over the energy rarige of the incident deuterium neutrals because of the variation of the electron emission coefficient of the detector surface with incident atom energy. Assignment of a kinetic temperature to the ion energy distribution is based upon the measured detector response as illustrated in Fig. A-5, Ref. 10. The selected calibration method requires a direct measurement of the microchannel plate response to a deuterium ion beam of known energy over the energy range from $50 \mathrm{eV}$ to $500 \mathrm{eV}$. We then assume, with some justification from the literature, that the neutral-deuterium atoms will produce an identical detector response as a function of energy ove- the same energy interval. A constant difference in detector response between the ion and neutral atom will not affect the calibration.

As much as possible of the time-of-flight neutral analyzer will be preassembled and tested. It will then be attached as a unit to the central vacuum chamber of TMX late in the assembly suhedule. This will reduce the time inierval between. he end of the construction phase and the acquisiiion of plasma data as well as ensure prompt assembly on TMX. We have acquired major portions of the vacuum system for the 2 -in flight path. The chopper motor is bein' vacuum tested and a snall ion source is being assembled for early tests of the microchannel plate detector. In addition to reviewing the physical principles of the diagnostic, we save completed the necessary engineering functions of cost estimation and scheduling. We have ordered long leaut-time items necessary to the primary TMX assembly.

\section{Mirror Fusion Test Facility (MFTF)}

As a result of major experimental successes in the LLL mirror program on startup and stabilization of plasmas in minimum-B magnetic geometry, a Mirror Fusion Test Facility (MFTF) was proposed. ${ }^{12}$ The MFTF will be used to bridge the gap between present-day small mirror experiments and future fusion reactor activity based on magnetic mirrors. The MFTF will investigate advanced engineering problems such as these associated with superconducting magnets, neutral-beam injectors, plasma-wall interactions, disposal of neutral particles and ions esca ${ }_{\mathrm{f}}$ ing from the plasma chamber, and high-speed vacuum pumping techniques.

Approval for t'le project has been given, and both operating and line item funding have been authorized for the MFTF. CGastruction of the facility began on Ottober I, 1977, with completion scheduled for July 1981. The operating budget activities includ $R \& D$ and fabrication and installation of apparatus for the first experiment in MFTF. The project is proceeding on schedule.

\section{Technical Support}

The objectives of the MFTF Technical Support Group are

- To monitor the MFTF design for consistency with the physics requirements.

- To provide researcil leading to improved performance of MFTF auxiliary components such as stmetup neutral-beam sources, plasma streaming sources, vacuum accessories, etc

- To analyze the physics of MFTF to ensure that the completed facility will achieve the stated scientific goals.

To keep the MFTF system requirements consistent with the current understanding of mirror fusion thysics. 
Neutral-Beam Requirements. / G. $D$ Porter. F. $H$. Coensien, R. S. Devoto, G. Leppelmeier, A. W. Molvik, J. E. Osher, J, W. Shearer, and $K$. I. Thomassen!

An assessment of the neutral bcam requirements indicates that 24 source modules will sustain the plasma as required.

Otir reassessment of the neutral-beam current requirements for the MFTF included an examination of the expected performance of the $80-\mathrm{keV}$ source operation based on the most recent results of development work at LBL. Because the mulecular mix of these sources is somewhat poorer than in the $20-\mathrm{keV} 2$ XIIB sources, the LBL source modules will produce more neutral current than previously anticipated, but the average beam energy will be 50 $\mathrm{keV}$ rather than $60 \mathrm{keV}$. We evaluated the current requirements for two plasma radial profiles and found that a diffuse (Gaussian) profile required more than 50 source modules to sustain a plasma with $\langle\beta\rangle=0.5$ and $\left[\left(a_{i} / n\right)(\partial n / \partial r)\right]^{-1} \geqslant 13$ over a volume that contains one-half of the piacna. The current requirements are somewhat more modest for a flat-topped profile. Then, a plasma with $\langle\beta\rangle$ $=0.5$ and $R_{p} / a_{i}=13$ can be sustained with 28 source modules. The 24 modules we expect to use will sustain a plasma with $\langle\beta\rangle=0.45$. This corresponds to a peak beta of $\beta_{0}=0.65$ and is considered adequate for the MFTF goals.

\section{Status of Continuing Developments}

Target-Plasma Production. (G. D. Porter)

We have continued the design of the fieldenhanced streaming-plasma gun that was discussed in the previous quarterly report. ${ }^{1}$ We found that the magnet described there was too large and hence too expensive to fabricate for the MFTF. A smaller magnet designed during the past quarter fits around the standard 2XIIB streaming-plasma gun, and the entire assembly can be inserted through a 6-in.-diam valve into the vacuum chamber. Detailed design of this assembly was completed and fabrication has begun. This field-enhanced streaming-plasma gun .will be tested in the 2XIIB.

Ion Beam-Dump Development. (R. C. Ling and A. W. Molvik)

We continued the study of beam dumps for the unneutralized ion beams. We have completed the development of a code that enables us to visualize the particle orbits in the fringing field of the MFTF magnet. We believe that the most probable beamdump configuration is a set of louvers that surrounds the neutral-beam chinnel. The dump must be made of louvers to permit pumping the gas that will cvolve from the surface. A code (GFUN) is being developed that will enable us to evaluate the ef- fect of the magnetic shielding on the ion trajectories.

Neutral-Beam Development. (A. W. Molvih)

The detailed design of the $: 0-\mathrm{keV}$ arc chamber and accelerator was completed. We are continuing a lower level design effort to correct problems found during the assembly and testing.

The arc chamber has been partially assembled to check that parts fit. Minor problems were found. These will be corrected and the arc chamber parts will be cleined and reassembled.

The accelerator parts were manufactured. The insulator-electrode stack was bonded together and finish-machined. Then, the gradient grid was assembled in the stack. The entrance grid was assembled, and the exit grid is ready to be assembled. The major delay in the accelerator is in obtaining the suppressor grid wire. LLL and Thermal Electron Corporatıon are working on wires in parallel. Both are close to the desired cross section, and the wires appear to be adequately straight. However, LLL found, after forming the wire, that the initial molybdenum stock had cracks. Therefore, the wire must be reformed using other wire stock. Thermal Electron must precurve the wire to a 7 -m radius, the produce enough wire for one source.

Vacuum Accessories. (F. Dixon)

Design was completed for the isolation valves, gimbals, bellows, support structure, neuiralizer duct, and high-voltage test stand (HVTS) adapters. Hardware delivery will start in April to provide the test equipment needed in interface the MFTF neutral-beam source with the HVTS. Investigation of magnetic stielding requir:ments led to a reduction in estimated shield weight. Optimization of the shield requirements was initiated with computer analysis for the MFTF neutral-beam injector arrays.

$80-k V$ Model. The development model of the MFTF 80-kV power supply was integrated and debugged. The accel dc power supply was assembled and malfunction of the variable-voltage transformer was corrected. The unit is ready for $480-\mathrm{V}$ ac low-voltage switching transient tests. Modulator tests were performed in March, with preliminary high-voltage testing on major subsystems of the accel switching modulator.

The gradient grid and dummy load were assembled. A seismic hazard analysis required design upgrade of the dummy load stand with work scheduled for early April. With the accel dc, modulator, gradient-grid, and dummy-load nearly complete, we plan development tests and integration with the sustaining neutral-beam source module for the next quarter.

Controls. Work in this area was mainly centered on research concerning an effective man-machine 
interface and on the development of software for automatic conditioning of the $80-\mathrm{kV}$ neutral-beam sources.

Research on the man-machine interface focused on two areas: touch panel graphics and console hardware layout. A microcomputer has been coupled to a color TV moniior and programmed to en abl z a user to generate and vary displays at will. It is being used to study various schemes for informatjon display and touch-panel control. To maximize operator ease and effectiveness, we have ordered a console cabinet in which display and control equipment will be configured in a variety of ways. These studies are leading to a control system philosophy based on human interaction with the proposed color touch-panel displays.

The sof'ware organization for controlling the 80$\mathrm{kV}$ model is complete. Software development is continuing. The $n$ cessary computer equipment began arriving in March: system debugging will begin in May whon the last components are delivered.

\section{Construction}

\section{(V. N. Karpenko)}

During the second quarter of the MFTF project, six of the scheduled key project milestones were completed to give a total of eight accomplished. A project review was held in January followed by detailed examination of the reestimated system and facility costs in February. Two major system procurements were initiated and design work started by two architectengineer firms, in addition to many smaller procurements. Removal and demolition progressed in Building 431. Some delays were experienced, but rescheduling of tasks covered most of the critical activities. Deferral of some noncritical efforts resulted in the rescheduling of the third milestone for award of a contract to Pacific Gas \& Electric Co. for the 2.30$\mathrm{kV}, 250-\mathrm{MW}$ power line with no major impact foreseen for the MFTF construction project.

Preliminary design efforts by LLL resulted in an increase in vessel size to provide more volume for cryopanels in order to increase vacuum pumping required for plasma stabilization with a gas box scaled up from the successful 2 XIIB experiments. Also, the increased distance to the ends of the ressel significantly reduced the end-loss energy flux. The distance was exter:ded from $6 \mathrm{~m}$ to $9 \mathrm{~m}$ from the cent or of the magnet to the inside of the vessel dome, a $50 \%$ increase.

The cryogenic system capacity was increased to provide the needed pumping speed with a revised arrangement of the cryopanels and an increase in surface area. The redesiga provided $500 \mathrm{~m}^{2}$ of panel array vs the earlier one-sided array of $340 \mathrm{~m}^{2}$. The helium liquifier capacity to support this subsystem was revised to $3000 \mathrm{~W}$. The liquid-nitrogen system usage rate was adjasted from 4700 to 5800 litre/h during MFTF operation. More specific project activities are identified beiow.

Magnet System. In February, we obtained results from the weld-sample tests of type 21-6-9 stainless steel that we plan to use in the magnet case. The filler metal was inconel 625 produced using the shielded metal-arc process. Two-inch plates were welded, and samples were sectioned for strength and toughness tests at liquid-helium temperature of $4 \mathrm{~K}$. We find thist the weld metal has good strength. ductility and toughness at $4 \mathrm{~K}$. The heat-affected zone has higher strength and lower but adequate toughness; the 21-6-9 base metal has higher strength and toughness. $:$ he test results provide positive support for the base material and filler metal for the magnet case. We are continuing to work on weld development to enahle selection of the best assembly techniques.

A rectangular cross section has been selected for the MFTF coil and we have chosen a supportsystem design.

A coil form for practice winding has been ordered. Fabrication of the superconductor core has begun, and we have selected a tension-control system for the coil winder. The superconducting test-coil winding was completed.

Design and procurement were completed for construction of the superconductor wraparound assembly line. The procurement package was forwarded to DOE/SAN for approval.

Our engineering studies on the magnet included seismic analysis and analyses of support structure, of case structure, and of the temperature gradients and stress resulting from cooldown. We also examined the possibility of reducing the thickness of the stainless-steel case from 3 to 2 in. in some areas; very favorable results include the promise of significant reduction in the weight of the magnet case.

Fusion Chamber System. In January, we sent a request for proposal (RFP) for the fusion chamber system to eight interested companies. A preproposal conference and site visit by interested companies resulted in clarifications and changes that were transmited in Amendment No. I to the RFP. dated March 13, 1978. Fiv. more amendments resulting froii additional questions were also issued in March.

We have begun to adapt a Monte Carlo code for calculating the effective pumping speed of the cryopanel subsystem for the fusion chamber system. Initial results, expected by mid-April, will provide analytical inputs to the technical evaluation of the 
proposals. One result will be an improved estimation of chamber pressures during gas injection.

The helium recovery compressors and helium storage hotlles now heing removed from the Nevada Test Site will all be delivered by the end of April.

The site of the cryogenic system has been shifted from the southwest side of Bldg. 431 to the northeast corner to allow access of fire-fighting equipment to another experiment area in Bldg. 431. This relocation greatly improved both component accessibility and flexibility of use.

Sustaining Neutral-Beam Power Supply System. The specification was developed for the MFTF sustaining neutral-beam power supply and reviewed by the 1.J.L. Electrical Engineering Department. The 80-kV system incorporated the design and development efforts mentioned above for the $80-\mathrm{kV}$ model. We have identified three different ways to meet the modulator-switch requirements for this system. These alternatives help reduce the implied risks to potential subcontractors. Further definition and tent activilies have increased our confidence in system designs with some cost reductions.

In April, personne! at Rome $\Lambda \mathrm{ir}$ Force Base will perform tests on the preferred tube for the switching modulator based on our established plans. The results are expected to provide design data for response to our RFP issued in March to indusiry: proposals are expected in June.

Control and Diagnostics System. Bids were opened in March for the MFTF computer system procurement. Seven proposals were received for the supervisory control and diagnostic system computers: five were received for the local control computers. We expect to complete our evaluation of these proposals by May 1978.

A rough draft of the preliminary operator's manual for the supervisory control and diagnostics system is $50 \%$ complete.

Starting with the control and diagnostics system, we are using a wide acceptance band, no-protocol interface. This interface choice reduces vendors' risk and provides for maximum standardization.

Software development work continued to support the line-item design efforts.

Bldg. 431 Modifications. A new detailed cost estimate was completed for Bldg. 431. Demolition work continued and is progressing according to plan. A project schedule has been received from the architect/engineer $(1 / E)$ for the Bldg. 431 modifications. The following relocations have been made:

- The control room and diagnostic area are being relocated at the northwest corner of Bldg. 431 to avoid the high magnetic ficld environment in the middle of the second floor area. We are studying the effect of this change on computer, peripheral equipment, experimental diagnostics, and central controi arrargements.

- Exterior cryogenic systems equipment will be located in Room 1060 of Bidg. 431.

- The pulse power substation providirg $480 \mathrm{~V}$ from the $230 / 13.8-\mathrm{kV}$ source will be located at the southeast corner of Bidg. 431.

Pulse Power. The final environmental impact statement for the $230-\mathrm{kV}$ power line was received from PG\& E and reviewed at L.L.L.

Desigr: work continued on the temporary power line. Interface definition with the power supplies was established. Work continued on design and planning of feeder transfers for power modifications to Bldg. 431.

The power-factor correction requirements were negotiated with PG\&E for the $230-\mathrm{kV}$ power contract. The factor range of 0.975 lagging to 0.85 leading for full load and 0.95 lagging to 0.80 leading for half load is considerably less stringent than the previous 0.99 for leading or lagging.

$\Lambda$ requisition for additional engineering prepared by $\mathrm{PG} \& \mathrm{E}$ is being reviewed by LLL.

Building 441. $\wedge / \mathrm{E}$ negotiations were completed, and an orientation meeting was held. Meetings are now being held with the LLL program staff. A required audit of the mechanical subcontractor has delayed the awarding of the A/E contract award to April.

Project Management. The MFTF Configuration Management Plan was prepared by LLL and ap. proved by the Project Manager on February 24th. Following review by the DOE San Francisco P:ojects office, the document was revised to provide additional detail on configuration approval and change control with in the LLL project and contractor efforts. Project level reviews of the specifications, work statements, terms/conditions and RFP instructions were zonducted prior to release of invitations for the fusion chamber system and for the magnet analyses and design proposals. Following a review of the procurement package for the sustaining neutral-beam power-supply package by the Livermore Project Office (LPO) Standing Review Committee and the LPO staff, the purchase requisition was signed and the RFP was issued. With issuance of the RFP, key milestone \#9 was completed on schedule.

The MFTF Performance Measurement System was implemented on MISTER, thereby providing a subsystem schedule network for the magnet system. 
Revised inputs were made to correct computer output and activity updates for the various systems. The magnet network was rerun with updated and corrected inputs. The other 'ask inputs are now being updated.

In the first Quarlerly Cost/Schedule Statizs Repor'. a management condition indicator was $: m$ ployed to silow the green. ye!low, or red status condition of each level 3 of the work breakdown structure element. These status ionditions were defined in the MF M Ifomhly Cost and Si hedule Report for Decenber 1977.

Activity during the quarter also included the following

- The HFTF Comingency Plan was approved by it: I! I. Project Manager and submitted to DU'..
- The preliminary Safety Plan issued in February was updated in March to include comments received from SAN. The improved Safety Plan will be issued as an MFTF document in April.

- The MFTF Qualir . Assurance Plan was issued in March, meeting the planned milestone.

The construcion activities are progressing with minimum variances in the key milestone schedules. Preliminary design activities have provided a sound basis for procurement planning. Detailed technical requirements evolved, with scme changes in systems designs previding improved confidence in meeting the overall experimental requirements. Design interration and interface defintion activities increased. Inputs from the superconducting test coil program and progress on the 80-kV power supply develupment model further enhanced the overall MFTF design base. 


\section{DEVELOPMENT AND TECHNOLOGY}

This program is aimed at developing the technology required for carrying out the mirror reactor program. Much of this work applies to the national program and fusion in general; it covers the following areas:

- Superconducting magnet development: The mirror program is the only fusion program that has depended upon superconducting magnets for major confinement experiments, and the only major confinement experiment presently under construction in the world using a superconducting magnet is the Mirror Fusion Test Facility (MFTF) at Lawrence Livermore Laboratory (J.L.L.). The magnel program is responsible for developing and lesting the conductor design for MFTF, as well as for developing, with industry's conperation. practical high-field materials, e.g $\mathrm{Nb}_{3} \mathrm{Sn}$, that will be needed for future experiments. $\mathrm{A}$ key feature of this program is the high-field test facility (HFTF) under construction in which the first large nultifilament $\mathrm{Nb}_{3} \mathrm{Sn}$ coils will be evaluated.

- Neutral-beam program (including beam direel conversion and vacuum technology): The reutralbeam program develops injector systcms for mirror experiments as well als for the Tokamak f usion Test Reactor (Tl-TR) and Doublet III. In addition to the development of prototype injectors for near-term experiments. a longer-range effort is aimed at improving reliability, quality. and efficiency of neutral-beam sources in general. $\Lambda$ major long-range effort is to develop high-efficiency, negative-ion sources for future high-energy applications. The 200-kV high-voltage test stand (HVTS). nearing completion. is a key elemert in future negative-ion-based beam development. In conjunction with work on neutral-beam injectors, we are also developing direcl energy converters for improving the efficiency of positive-ion-based systems. and we are developing the advanced eryopumping techniques that are needed for all large neutral-injector systems

- Direct conversion: In addition to our study of the direct conversion associated with neutral beams. we have a continuing program to develop efficient direct recovery systems. which are required for reducing power losses from future mirror reactors.

- Materiais program: We have a unique source of $14-\mathrm{MeV}$ neutrons at the Rotating Target Neutron Source (RTNS-I), an LLL facility that is used to investigate the effects of fusion neutrons on materials of interest to reactor designers. Specimens are irradiated for evaluation by many laboratories. A new facility dedicated to the fusion program, the RTNS-II, is nearing completion. The LLL materials program is ajmed at characterizing the type of damage effects produced by fusion neutrons and correlating these effects with fission reactor experiments.

Several key problems on tritium control and handling that must be solved for any large D-T fusion device are being investigated in the LLL tritium laboratory; emphasis is on cleanup of low tritium concentrations in reactor centainment buildings and on the containment of tritium by using various low-permeability barriers and coatiness to be applied to metal walls.

The effects of neutrons on the properties of superconducting materials are being investigated using a unique apparatus in which superconducting properties are measured while the specimen is continuously maintained at Jiquid-helium temperature.

- Reactor design studies: Design studies of mirror reactors form a basis for evaluating mirror concepts and for guiding our long-range program. Present emphasis is on delineating features of reactors based on the tandem mirror reactor (TMR) concept, on a fission/fusion hybrid reactor based on the TMR, and on an engineering evaluation of a small reactor system based on field reversal. A special class of reactors that are small and candidates for const:uction in the next decade is being investigated in a program sponsored by the Electric Power Research Institute (EPRI).

\section{Magnet Systems}

Superconducting Magnet Development (D. N. Cornish)

The technology of superconductors and superconducting magnets is of vital importance to the building of present-day plasma physics facilities and to the planning and study of future experimental mirror machines and reactors. We are developing a $\mathrm{Nb}-\mathrm{Ti}$ conductor for the Mirror Fusion Test Facility (MFTF) winding and $\mathrm{Nb}_{3} \mathrm{Sn}$ conductors for future mirror devices.

MFTF Development. We have completed the winding of the test coil for the Mirror Fusion Test Facility (MFTF) using 6,500 ft of prototype conductor. Valuable experience was gained in learning 
how to wind and join this conductor. The coldwelding of the core presented no problems, and all the joints appeas to be sound. However, we encountered problems in soldering the stabilizing jacket over the joined core, principally because the latter had a strong tendency to curve as soon as the jacket was removed. The problems were overcome by modifying the splicing arrangements of the jacket and by twisting the conductor so that its natural direction of curvature matched that of the winding.

Strip heaters of lengths varying from a few centimetres upwards were attached to three adjacent layers so that the equivalent of about 1-1/6 turns of the magnet winding carried a heater. These heaters are designed to enable us to investigate the propagation and recovery characteristics of initial normal zones, which vary in size from a few centimetres of a single turn to several full turns. Several joints were also fitted with heaters so that their performance could be studied and related to that of the main body of the windings. Strain gages were also incorporated, largely to check their performance under these operating conditions.

The completed coil was then assembled between two similarly sized coils that had been wound previously for the high-field test facility (HFTF). Fig. 19 shows the coil assembly, with its array of diagnostic leads, about to be coupled to the topplate assembly of the cryostat. The main and diagnostic wiring has been completed, and the whole assembly is ready for lowering into the $2-\mathrm{m}$ diam cryostat for its first cool-down.

We have completed the fabrication and installation of associated equipment, including lifting facilities for 10 tons, power supplies, control and protective systems, and an extension to the heliumrecovery system; cool-down is scheduled to commence at the beginning of April.

High-Field Test Facility. The power supply, protective systems, and controls required to operate the first two sections of $\mathrm{Nb}-\mathrm{Ti}$ backing coils together with the MFTF test coil have now been fabricated and installed and are ready for commissioning.

The superconductor ordered from Supercon for the remaining two backing coils is late; we now expect the first consignments to arrive in early April.

$\mathrm{Nb}_{3} \mathrm{Sn}$ Conductor Development. Airco is proceeding with the development and fabrication of a prototype length of stabilized conductor as outlined in Ref. 13. The first-stage billet consisis of a 188-mm finished diameter, $13 \mathrm{wt} \% \mathrm{Sn}$ bronze casting, drilled with $1877.04 \mathrm{~mm}$-diam holes into which the $\mathrm{Nb}$ rods will be inserted. This bronze billet was vacuum-induction melted and cast on a previous development contract; when machined and drilled, it was found that internal porosities connected the internal seven or eight holes in the central region of the upper half of the billet. The billet was therefore given two hot-vacuum bakes to remove any residues from the machining and drilling operations before the $\mathrm{Nb}$ rods were loaded into it. This villet is scheduled for extrusion duridg the first week in ipril.

I he second-stage billet will consist of a $250.2-\mathrm{mm}$ o.d., 142.6-mm i.d. copper billet loaded with 1777 hexagonal rods drawn from the first-stage billet. $A$ two-piece sheet-Ta liner with overlapped edges will separate the "hex-pack" from the copper can.

Because the hex-pack is surrounded by a thick, relatively soft $\mathrm{Cu}$ layer. there is a danger that "center-bursting" might occur during the extrusion process unless parameters such as die angle are exactly correct. There could also be problems associated with the wrapped Ta barrier, and there is some doubt about whether annealing is required after each or after every two passes through the drawing dies. To check these uncertainties and to make any necessary modifications before the expensive billet is processed, a dummy billet has been processed ahead of it. This billet consists of a central $13 \mathrm{wt} \% \mathrm{Sn}, 138.2$-mm-diam, 552-mm-long bronze rod that is wrapped with $\mathrm{Ta}$ sheet and loaded in to a $250.2-\mathrm{mm}$ o.d., 142.6-mm i.d. Cu can. The billet has been extruded and to date has been drawn down to a $32.5-\mathrm{mm}$ diameter. Although detailed examinations have not yet been carried out, it appears to be satisfactory. Processing will conunuc to the finished size of $5.4 \times 11.0 \mathrm{~mm}$, and detailed evaluation will be made. It will then be used for trial runs to check the proposed method of adding the stabilizer.

During 1977, Supercon attempted to scale up their Nb tube/bronze process from 2-in.- to 8-in.diam billets. The first-stage billet was successfilly extruded, and samples were drawn down to produce satisfactory 0.027 -in.-diam wire. However, many irregularities and broken filaments were found in the extruded rod from the second-stage billet. There is some doubt as to the reason for this failure. Possible causes include faulty control resulting in too low an extrusion temperature, possible loss of vacuum in the billet due to a fall during shipment, or too great an extrusion reduction ratio.

The total cost of developing any process to the final goal-the economical fabrication of filamentary $\mathrm{Nb}_{3} \mathrm{Sn}$ conductor on a large-scale production basis-is very substantial. Furthermore, the spending rate accelerates as the billets increase in size and cost. Since the overall funding for this development activity is strictly limited, its allocation is continually under review. During FY 1977, we 


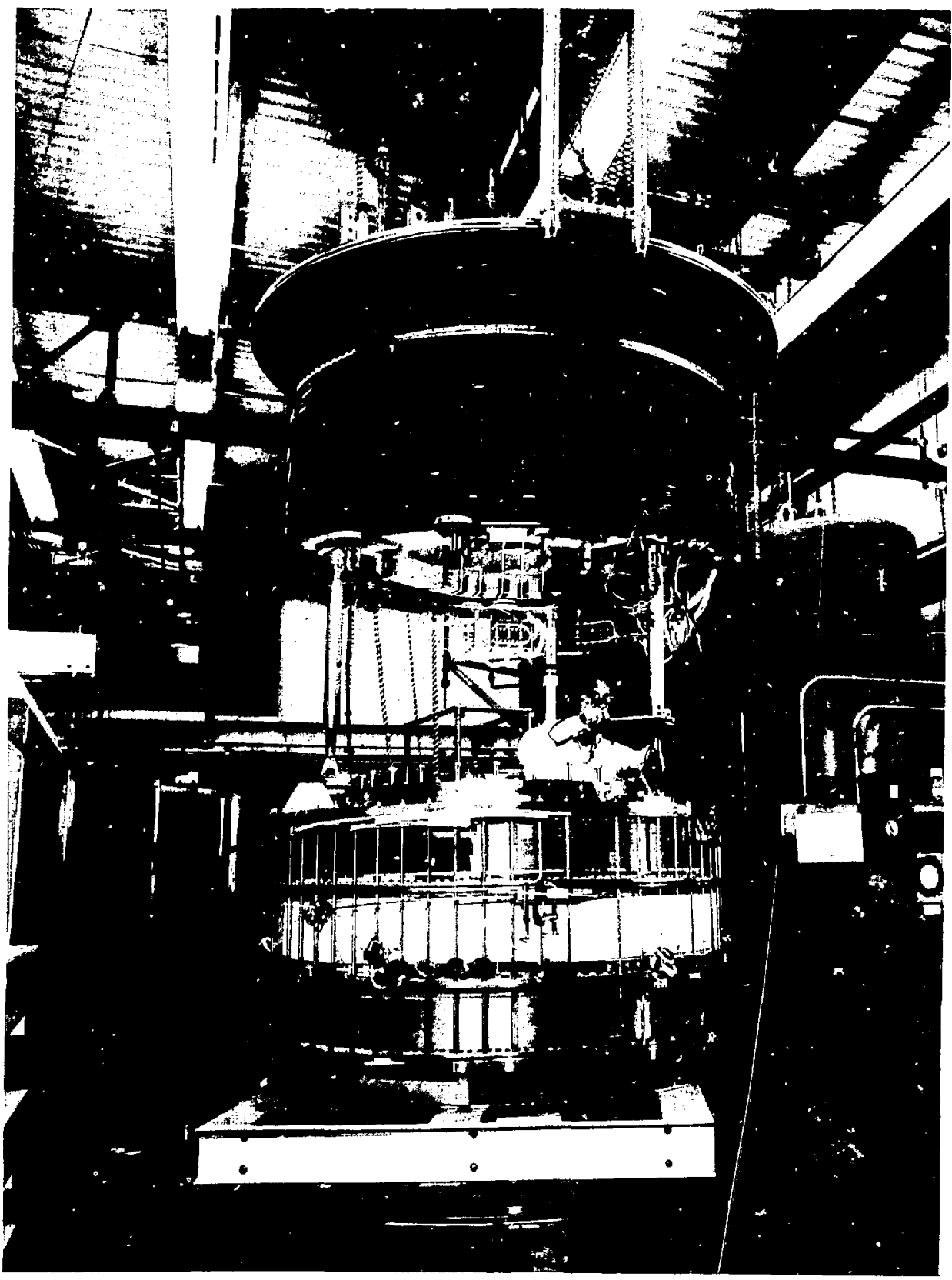

Fig. 19. MFTF test coil assembled between coils for the High-Fïeld Test Fucility. 
had shown that the strain characteristics of the bronze-matrix $\mathrm{Nb}_{3} \mathrm{Sn}$ conductor were acceptable for the large fusion magnets. It was at that time, therefore, considered to be in our best inicerest to concentrate on the further develonment of that conductor. However, although we plan to carry out strain measurements on samples previously supplied by Supercon during the next quarter, we do not propose to continue to fund the develupment of this Supercon type of conductor.

The preliminary study at M.C.A. to assess the feasibility of scaling up the powder metallurgy approach to the fabrication of commercial $\mathrm{Nb}_{3} \mathrm{Sn}$ filamentary conductors has continued. A $25-\mathrm{mm}$ diam $165-\mathrm{mm}$-long billet containing six Ta-clad, $\mathrm{S}_{\mathrm{n}}$ infiltrated, $11.7-\mathrm{mm}$-diam sintered-Nb rods was extruded to a $6.2-\mathrm{mm}$ diameter. This rod was severely fractured both internaliy and externally. We believe that this poor ductility resulted from the relatively poor vacuum $\left(\sim 5 \times 10^{-5} \mathrm{Torr}\right)$, which was the best that could be achieved during the sintering; a value of $10^{-6}$ Torr or lower was used during the preliminary work at $\mathrm{LBL}$.

\section{Plasma Engineering}

\section{LLL/L.BL Neutral-Beam Development (E. B. Hooper and R. V. Pyle)}

The LLL/LBL Neutral-Beaın Development Group ibis the responsibility for developing neutral-beam injectioi: systems for mirror and tokamak experiments. Modules with beam energies of 20 to $40 \mathrm{keV}$ have been used on the 2XIIB and Adiabatic Toroidal Compression (ATC) devices. Current near-term developinent efforts are directed toward $80-$ to $120-k V$ injectors for the MFTF (80-kV), Doublet III (D-III) (80$k V$ ), and Tokamak Fusion Test Reactor (TFTR) $(120-k V)$ experiments. The total beam power from each accelerator module will be 6 to $7 \mathrm{MW}$, in 0.5-s pulses. The users want to place orders for production models in about 1 year. Development goals for the longer term are near steady-state operation, iıgher energy, and better efficiency; this part of the program is centered on negative-ion production, acceleration, and neutralization. We hope to have 200 - to $490-k \mathrm{~V}$ injectors based on negative ions developed in time for application in the mid- to late-1980's.

Development for near-term and mid-term applications (based on positive-ion technology) continues to dominate our efforts. Although there is still much quantitative work to do on the TFTR, MFTF, and DIII injectors, we have made sufficient progress in the past quarter to give us considerable confidence that it is technically possible to meet the development goals for these experiments.
Positive-Ion Program. Our positive-ion-based work had the following highlights:

The full-scale TFTR prototype ion source (basically similar to MFTF $a:$ i D-III sources) operated on test stand IIIB (TS i:I / s) .t $100 \mathrm{kV}, 70$ $A$ in a short-pulse ( $15-m s$ ) mode with hydrogen. The goal is $120 \mathrm{kV}, 65 \mathrm{~A}, 0.5 \mathrm{~s}$ with delterium. (Most of our testing is with hydrogen $b$, cause of neutron problems associated with deuterium use.)

'Testing for the full pulse length will be done on the HVTS facility later in the year, after we have demonstrated 120-kV. 63-A, 23-ms operation with deutcrium on TS IIIB.

We have built a "magnetic bucket" plasma source having a square cross section suitable for fractional a ea $(\sim 15 \mathrm{~A}) 80$ - to $120-\mathrm{k} V$ source operation and ha! 9 measured its plasma properties. The external surface of the arc chamber is covered with suitably positioned permanent migncts that produce a multipole field within the are chamher. The magnetie field slows the loss of charged pisticles from the plasma to the chamber walls. On the basis of our previous measurements with a cylindrical magnetic bucket and on experience at Oak Ridge National Laboratory (ORNL) with cylindrical buckets, we expect that this new plasmal source should have a higher fraction of atomic ions than our present sources have. Preliminary operation the last day of the quarter (with hydrogen) on a fractional-area TFTR source confirmed that the atomic fraction is indeed higher, but we have so far obtained little quantitative data.

Draper Lab personnel successfully demonstrated fully-automated runup of a newl. assembled $80-\mathrm{kV}$ source.

Beam-plasma interactions can occur in the néutralizer of a neutral-beam system, possibly affecting the optical properties of the beam in an adverse way. Optical and Langmuir-probe measurements in the neutralizer of our $120-k$ V TS 111 system indicate that some such interaction may be taking place, and that the beam divergence may be approximately doubled as a result. Because the final neutral bearn still meets the specifications of present users, this potential problem will be investigated with low priority.

An energy-recovery try by the direct-recovery group was attempted with a $120-\mathrm{kV}$ injector on TS IIIA. Poor vacuum caused by beam-surface gas evolution prevented a successful demonstration; they will try again during the next quarter.

Studies of possible radiation effects on cryocondensation pumping systems were started at the U.C. Eerkeley Triga fission reactor.

Negative-Ion Program. Negative-ion workers pursued two paths: 
- Continuation of the work to produce a negative-ion beam by accelerating positive ions to low energies, passing them through cesium vapor to convert a fraction to negative ions, and then accelerating the negative ions to high energies. Most of the effort was devoted to developing better lowenergy positive-ion sources, both conventional accelerators and energetic arcs. Construction began on the cesium cell to house the new cesium jet.

- Development of alternate techniques for producing negative ions, e.g., production on surfaces. Most of our studies during this quarter werc concerned with surface production.

Facilities. The high-voltage power supply fo: the HVTS was operated at $60 \mathrm{kV}$. Also, we completed plans for modifying TS IIJA for 2-s operation.

\section{Direct Conversion}

Beart Direct Energy Conversion. /W. L. Barr. $(j$. W. Liamilton, and R. W. Moir I

Apparent discrepancies hetween results from two tests of the beam direct converter are tentatively ascribed to desorption of gas from the electrodes and heam dump.

The most recent test (February 1978) of the beam direct converter on the $120-\mathrm{keV}$ beam line at LBL was done with $\mathrm{He}^{+}$ions to avoid molecular (and hence fractional-energy) ions. The results with either magnetic or electrostatic electron suppression difsered only slightly from earlier (October 1977) results. The main difference was that the initial selfbias on the collector did not stop at $40 \mathrm{kV}(1 / 3$ energy) as before, but got as high as $75 \mathrm{kV}$ under some conditions (e.g., when the collector was floating). The collector current and voltage decreased to zero within $300 \mathrm{~ms}$ after the initial fast rise. The maximum average collector current was $0.37 \mathrm{~A}$, while the net electrical current decayed from a maximum initial value of $0.3 \mathrm{~A}$. These results indicate that the cancelling electron current increased during the pulse as the gas density increased. The maximum collection efficiency is about $10 \%$ at the beginning of a pulse.

We believe we have now identified the cause of the discrepancy as being the desorption of gas from the electrodes and possibly also from the LBL beam dump. A Langmuir probe located near the first aperture indicated a cold plasma with $\mathrm{T}_{\mathrm{e}} \approx 4 \mathrm{eV}$ and a density that increased to $2 \times 10^{9} \mathrm{~cm}^{-3}$ by the end of a pulse. A gas analyzer showed that $\mathrm{H}_{2}$ is produced in the direct converter at a rate comparable to that of He from the beam. The beam is pure $\mathrm{He}$, but $\mathrm{H}_{2}$ might be selectively reabsorbed between shotis from the gas mixture that exists in the LBL vacuum system. The vacuum system is shared with beam line IIIB, which operates on hydrogen.
We are working on solutions to the outgassing problem tbat appears to be common to both electrostatic and magnetic electron suppression.

\section{Reactor Materials}

RTNS-II

\section{i.). L. Osher. (: Ilanson and J. C. Davis!}

The R T.VS-II facility will provide the neutrons required to determine the response of potential fusion reactor materials to $14 . \mathrm{MeV}$ neutron irradiation. Areas of research to be explored with this machine include radiation effects on superconductors, damagerate studies, changes in observahle microstructure, and changes in physical properties. A major emphasis of this work will be to develop the knowledge and methods required to relate data from other radiation sources to the fusion-neutron environment. We anticipate some effort in measuring neutron and gas production cross sections.

During the second quarter of FY 1978, we met two major goals of the RTNS-II project:

- The prototype accelerator became fully opcrational for tests with ion beams, and

- The project staff moved into the newly completed Building 292 to begin installation and assembly of the neutron source components.

The acceleration column is snown in a close-up view in Fig. 20 and installed on the prototype accelerator in its test enclosure in Building 212 in Fig. 21. Without beam, the acceleration column has been tested to its full operating voltage of $380 \mathrm{keV}$; with $\mathrm{H}_{2}^{+}$beam, operation has been limited to $\sim 30$ to $40 \mathrm{~mA}$ at energies up to $250 \mathrm{keV}$ by the lack of radiation shielding around the accelerator. A rotating target is now being installed on the prototype for target tests. All accelerator diagnostic and control sysiems have been tested successfully.

With the start of installation activities in Building 292 in March, initial operation of the first source in Building 292 is expected in July 1978. Experimenters will have access to the RTNS-11 Facility at that time to develop irradiation experiments.

\section{4-MeV Neutron Irradiation Studies}

Radiation Damage In Superconductors. $(R . M$. Scanlan)

In designing the magnet shielding for magnetic fusion reactors now in the planning stages, we must know the effects of radiation on candidate superconducting materials as a function of fluence. Calculations for a tokamak reactor predict a total neutron nux of between $4 \times 10^{12}$ and $4 \times 10^{13} \mathrm{n} / \mathrm{m}^{2} \cdot \mathrm{s}$ at the superconductor. These flux calculations are based on perfect shielding; as more refined calculations are 


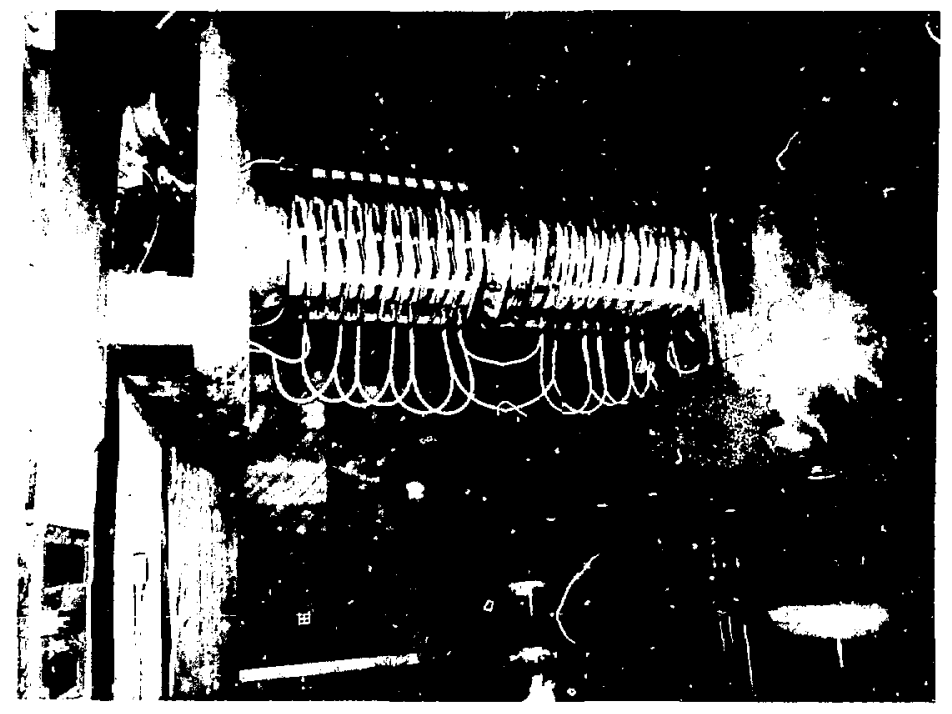

Fig. 20. RTNS-1] acceleration column.

made that incorporate neutral-beam injection ports, etc., the flux values at localized areas of the magnet will increase. Because the magn:ts must be used for many years, the radiation tolerance of the magnets may become the controlling factor.

The work in progress has the following three objectives:

- Determination of the effects of neutron irradiation (from about $4 \mathrm{MeV}$ to $14 \mathrm{MeV}$ ) on the critical current of $\mathrm{Nb}_{3} \mathrm{Sn}, \mathrm{Nb}-\mathrm{Ti}$, and other superconductors at liouid-He temperatures and in the presence of magnetic fields up to $12 \mathrm{~T}$,

- Correlation of the electrical properties of these materials with both initial microstructure and radiation-induced changes in microstructure, and

- Measurement of the recovery of the changes in the critical current during annealing at temperatures up to room temperature.

During the past quarter, $\mathrm{Nb}-\mathrm{Ti}$ superconductor samples were irradiated and results were compared with those from an earlier test. Critical current inconsistencies must be resolved in future tests.
Our previous results on radiation damage in $\mathrm{Nb}$ $\mathrm{Ti}$ (see Ref. 4) showed some unusual behavior in that upon annealing after irradiation the critical current first decreased and then recovered at higher temperatures. To substaniiate this behavior, we repeated the experiment at the RTNS-I.

Two samples of $\mathrm{Nb}-\mathrm{Ti}$ superconductor were irradiated to a fluence of $8 \times 10^{20} \mathrm{n} / \mathrm{ri}^{2}$ at the RTNS-I. One sample had been irradiated previously to a lluence of $8 \times 10^{20} \mathrm{n} / \mathrm{m}^{2}$, and the other sample was unirradiated. The results of this most recent irradiation were totally unexpected: the critical currents of both samples remained unchanged during the irradiation, whereas we had measured a $26 \%$ decrease in critical current at the same fluence in the previous experiment.

To evaluate differences between the two irradiations, we examined the autoradiography to check sample alignment during the irradiations and the dosimetry to determine the neutron fluences: there were no significant differences. At this time, we conclude that the most recent experiment (which 


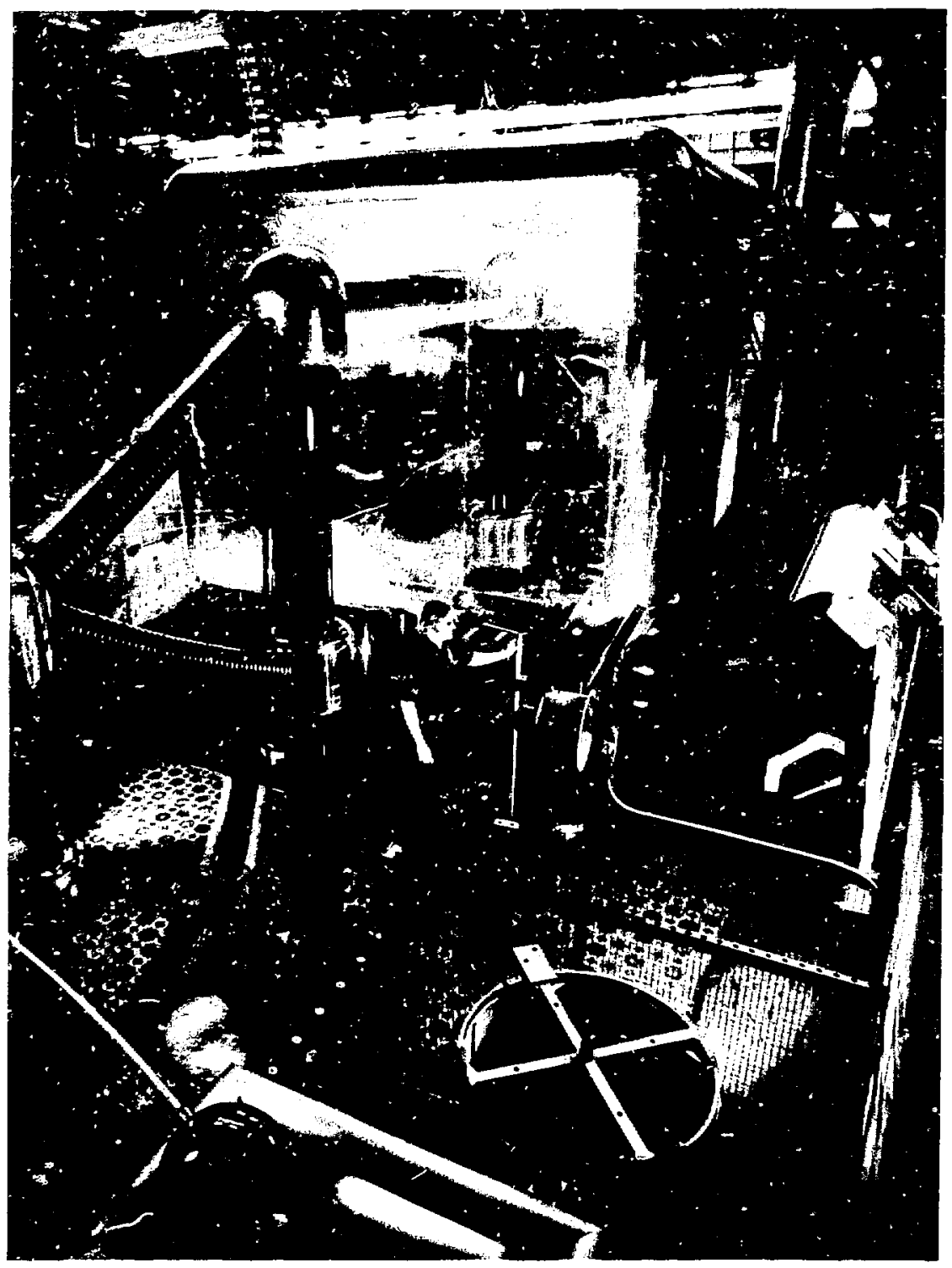

Fig. 21. RTNS-II power supply and high-voltage enclosure. 
showed no change in critical current to a fluence of $8 \times 10^{20} \mathrm{n} / \mathrm{m}^{2}$ ) is correct. One possible explanation for the observation of a decrease in critical current in the previous irradiation is that the thermal contact between the specimens and the copper block changed with irradiation and the specimen temperature therefore increased during the irradiation. However, because there are no data available on the effects of irradiation on thermal conductivity of organic insulation, we cannot at present verify this explanation.

The best way to resolve the differences between these experiments is to continue the irradiation to higher fluences and to measure the critical current change with fluence. This experiment will be performed at the U.C. Davis $\left.\mathrm{Be}^{\prime} \mathrm{d}, \mathrm{n}\right)$ source as soon as funds are available.

Effect of Helium on Mechanical Properties of $\mathrm{Nb}$ and $\mathrm{Nb}-1 \% \mathrm{Zr}$. (W. L. Barmore and R. R. Vandervoort!

The mechanical properties of materials used in fusion reactors are changed by large concentrations of internally generated helium. Because of high $(n, a)$ cross sections, significant quantities of helium will be produced in fusion reactor materials irradiated by 14$\mathrm{MeV}$ neutrons in a relatively short tire span. For example, a concentration of 25 to 50 atomic ppm helium will be produced internally in niobium by a fusion neutron flux of $\sim 10^{18}$ neutrons $\mathrm{m}^{-2} \mathrm{~s}^{-1}$ with 1 year's exposure. Helium atoms produced at a high concentration in a host matrix having low helium solubility are thermodynamically unstable and have a high driving force to coalesce in the microstructure at various defects. Thus, helium atoms tend to migrate to dislocations, precipitates, grain boundaries, impurity particles, and microcracks and to coalesce into bubbles. These helium-induced defects generally cause embrittlement.

We have developed an optimized process for doping $\mathrm{Nb}$ and $\mathrm{NB}-1 \% \mathrm{Zr}$ specimens with helium using the tritium-decay method. Tritium is dissolved in the metal at $400^{\circ} \mathrm{C}$ to give a resultant $\mathrm{Nb}$ to ${ }^{3} \mathrm{H}$ ratio of 20 and an equilibrium partial pressure of $1.33 \mathrm{~Pa}$ over the specimens. The tritium decays by the reaction ${ }^{3} \mathrm{H} \rightarrow{ }^{3} \mathrm{He}+\beta^{-}$at a rate that produces about 7 atomic ppm helium per day in the host microstructure. Using this technique, we can successfully dope specimens to 500 atomic $\mathrm{ppm}{ }^{3} \mathrm{He}$ in less than 10 weeks. During the past quarter, we determined the tensile properties of $\mathrm{Nb}-1 \% \mathrm{Zr}$ with helium concentrations of 0 to 100 atomic ppm and ai temperatures up to $800^{\circ} \mathrm{C}$.

We are investigating helium effects that are separate from combined helium and displacementdamage effects by doping $\mathrm{Nb}$ and $\mathrm{Nb}-1 \% \mathrm{Zr}$ specimens with ${ }^{3} \mathrm{He}$ by the tritium-decay method to as high as 500 atomic ppm and studying changes produced in the mechanical properties.

The tensile properties of $\mathrm{Nb}-\mathrm{l} \% \mathrm{Zr}$ at temperatures up to $800^{\circ} \mathrm{C}$ as a function of helium concentration are given in Table 4. The strengthening effects and losses in ductility caused by helium are

Table 4. Tensile tests on $\mathrm{Nb}-1 \% \mathrm{Z}_{\mathrm{r}}$.

\begin{tabular}{|c|c|c|c|c|c|}
\hline $\begin{array}{c}\text { Helium } \\
\text { (atomic } \\
\text { Ppm) }\end{array}$ & $\begin{array}{c}\text { Test } \\
\text { temperature } \\
\left.{ }^{\circ} \mathrm{C}\right)\end{array}$ & $\begin{array}{c}\text { Yield stress } \\
(\mathrm{MPa})\end{array}$ & $\begin{array}{c}\text { Ultimate } \\
\text { stress } \\
\text { (MPa) } \\
\end{array}$ & $\begin{array}{c}\text { Elongation } \\
(\%)\end{array}$ & $\begin{array}{c}\text { Reduction } \\
\text { in area } \\
(\%)\end{array}$ \\
\hline 0 & 23 & 150 & 260 & 53 & 95 \\
\hline 25 & 23 & 165 & 279 & 46 & 95 \\
\hline 100 & 23 & 234 & 355 & 36 & 65 \\
\hline 0 & 200 & 106 & 202 & 43 & 95 \\
\hline 25 & 200 & 92 & 194 & 45 & 95 \\
\hline 100 & 200 & 158 & 275 & 30 & 85 \\
\hline o & 400 & 102 & 202 & 27 & 95 \\
\hline 25 & 400 & 92 & 194 & 33 & 95 \\
\hline 100 & 400 & 147 & 244 & 30 & 75 \\
\hline 0 & 600 & 70 & 222 & 32 & 95 \\
\hline 25 & 600 & 85 & 208 & 31 & 95 \\
\hline 100 & 600 & 116 & 235 & 25 & 55 \\
\hline 0 & 800 & 65 & 204 & 31 & 95 \\
\hline 25 & 800 & 78 & 221 & 27 & 65 \\
\hline 100 & 800 & 185 & 292 & 12 & 30 \\
\hline
\end{tabular}


pronounced, particularly at a concentration of 100 atomic ppm. Note the loss in ductility at $800^{\circ} \mathrm{C}$ in the specimen containing 100 atomic ppm helium. The combined effect of temperature and deformation may lead to the rearrangement of helium in the metal matrix, the result being deleterious changes in the microstructure. Electron microscopy observations showed a homogeneous distribution of helium in as-charged specimens at 25 atomic ppm, however, as the helium concentration increased, there was a greater tendency for helium bubbles to form on grain boundaries.

\section{Fusion Systems Engineering}

\section{Reactor Design Studies.}

Performance Parameters for Fusion-Fission Power Systems. (D. J. Bender)

A comparatively near-term application of presentday fusion research, the hybrid (or fusion-fission; reactor, has recently received increased attention because of its potential to serve as a supplier of fuel to the nuclear power industry. ${ }^{14-16}$ Because the hybrid produces a source of neutrons external to the fission process, it is a highly nexible nuclear breeding option that permits the use of a wide variety of nuclear fuel cycles.

We have developed goals for the following parameters: plasma power amplification and hybrid unit capital cost ratio.
Fortesque ${ }^{16}$ recently proposed a set of parameters that could be used to compare the fissile breeding performances of the hybrid and the fast breeder reactor. We have elaborated on the analysis of Fortesque to reflect considerations that we have found important in our studies of the hybrid reactor. Our objective is to evolve a set of general performance parameters that will permit comparison of diverse hybrid reactor designs and fuel cycles on a common basis. Here, we present a summary of the full analysis. ${ }^{17}$

The fusion-fission symbiosis under consideration is represented in Fig. 22. We assume a driven fusion reactor that produces a gross electrical power $\mathrm{P}_{\mathrm{eb}}$ (based on one unit of electrical input power to the plasma heating equipmerit). The hybrid also produces fissile fuel to provide fuel makeup requirements for a group of fission converter reactors having electrical output $P_{c c}$. The model used for the hybrid power flow is diagrammed in Fig. 23. We considered two hybrid configurations:

- "On-line" operation in which the thermal energy from the hybrid is converted to electrical output, and

- "Fuel factory" operation in which all thermal energy from the hybrid is rejected $\left(\eta_{\mathrm{tb}}=\mathrm{P}_{\mathrm{eb}}=0\right)$.

We propose the following parameters to describe the fusion-fission power system:

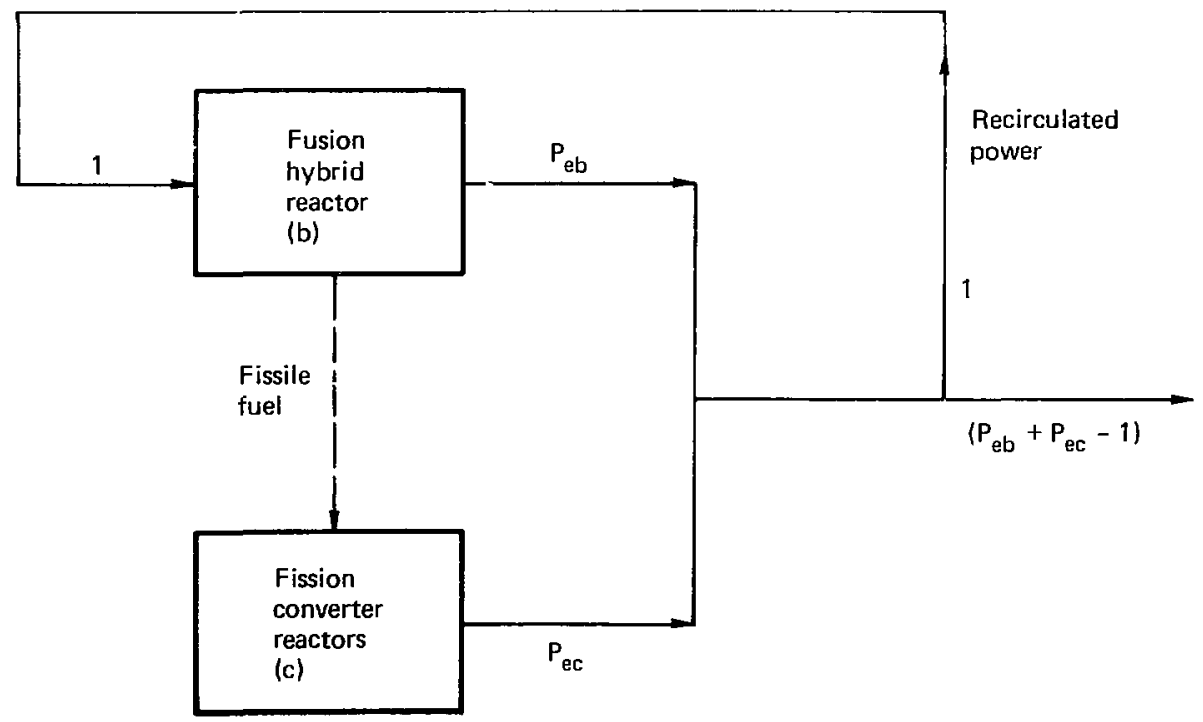

Fig. 22. Electrical power flow for the entire fusion-fission system. 


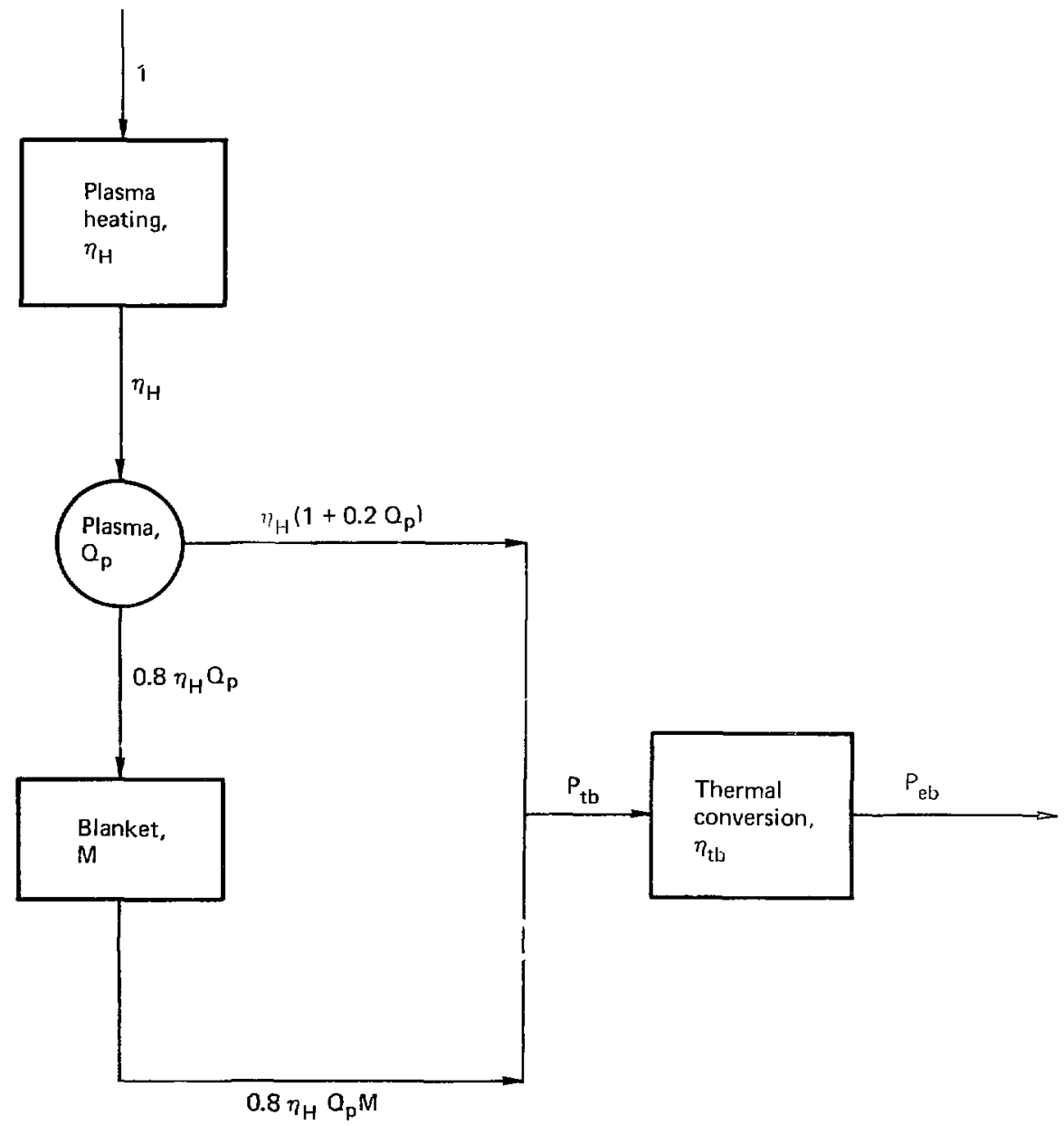

Fig. 23. Power flow in the fusion reactor.

- Fusion power multiplication in the converters:

$$
r=\frac{\text { converter thermal power }}{\text { fusion power }}
$$

- Thermal power ratio:

$$
R_{1}=\frac{\text { converter }}{\text { breeder }} \frac{\text { thermal nower }}{\text { thermal power }}
$$

- Electrical energy multipiication

$$
Q_{E}=\frac{\text { system gross electrical output }}{\text { breeder electrical input }}
$$

- System unit capital cost ratio

$$
R_{c}=\frac{\$ / k W(e) \text { for system }}{\$ / k W(e) \text { for converter }}
$$


which is expressed as a function of the breeder unit capital cost ratio

$$
k=\frac{\$ / k W(t) \text { for breeder }}{\$ / k W(1) \text { for converter }}
$$

Expressions for these performance parameters are derived in terms of the power-flow parameters appearing in Figs. 22 and 23.

We have considered two examples, the first based on the $\mathrm{U} / \mathrm{Pu}$ fuel cycle and the second on the $\mathrm{Th} /{ }^{2.33} \mathrm{U}$ fuel cycle. Power-flow parameters were selected from the results of our hybrid reactor studies, ${ }^{18.19}$ and the formulation was used to develop target performance parameters for plasma power amplification $Q_{p}$ and for the hybrid unit capital cost ratio $k$.

The variation of the system unit capital cost ratio is shown in Fig. 24 as a function of the plasma power amplification. Here, the Th/ $\mathrm{L}$ fuel cycle is used with the hubrid in the "on-line" configuration. The unit capital cost ratio for the hybrid $\kappa$ is treated as a parameter that varies from one to three. The following significant information is displayed on this figure:

- For $Q_{p}=1$, the system capital costs are within 10 to $15 \%$ of the asymptotic values achieved with an ignition plasma $\left(Q_{p}=00\right)$, thus demonstrating the adequacy of $Q_{p}=1$ to 2 for hybrid reactor applications; and

- Even for hybrid capital cost ratios as high as $\alpha$ $=3$, altractive system capital costs can be achieved $\left(R_{c} \geq 1.25\right)$ with modest plasma performance.

The following results are obtained from the two examples:

- Scientific feasibility, or breakeven $\left(Q_{\mathrm{E}}=1\right)$, occurs for the fusion-fission power system at $Q_{p}$ $\approx 0.1$.

- If we select $R_{c}=1,25$ as an economically competitive value, this condition is achieved by the on-line hybrid for plasma power multiplication of $Q_{p} \approx 1-2$ and hybrid unit capital cost ratios of $\kappa$ $\approx 1.5-2.5$.

- The fuel factory configuration, at the same value of $Q_{p}$ as the on-line hybrid, must have a capital cost reduction of $\sim 2$ to achieve comparable economics.

The Field-Reversed Mirror Reactor. (G. A. Carison)

It may be possible to sustain a field-reversed, mirror-confined plasma using neutral beams. This would make possible small fusion reactors. We have completed a design study showing how several fieldreversed cells can be assembled to make a commercial reactor.

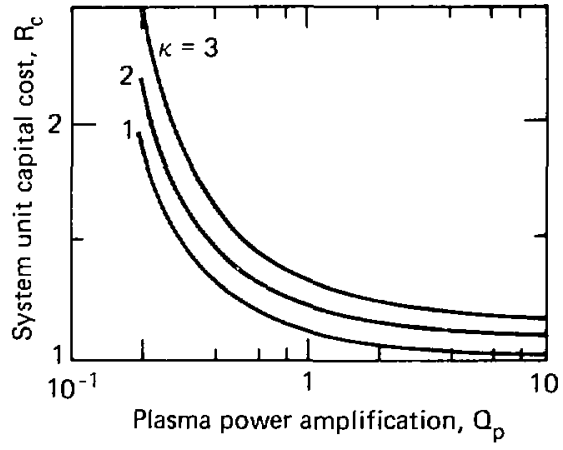

Fig. 24. Variation of system unit capital cost ratio as a function of the plasma power amplification for a Th/t cycle and on-line operation, where $k$ is the capital cost ratio for the breeder unit.

The reference case field-reversed mirror (FRM) reactor ${ }^{20}$ is a multicell configuration with 11 field. reversed toroidal plasma layers arranged along the horizontal axis of a long superconducting solenoid (see Fig. 25). Each plasma layer requires the injection of $3.6 \mathrm{MW}$ of $200-\mathrm{keV}$ deuterium and :ritium and produces $20 \mathrm{MW}$ of fusion power. The reactor has a net electric output of $74 \mathrm{MW}(\mathrm{e})$.

The parameters of the reference case design result in part from somewhat arbitrary physics assumptions and in part from optimization procedures. We constructed an analytic model of the FRM reactor, encompassing physics, engineering, and economics, to permit investigating the effects of various assumptions and parameter variations. The optimization procedures were directed towards minimizing the estimated direct capital cosi divided

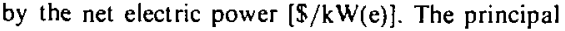
parameters that were optimized were the injection energy and the reactor cell length. The estimated direct capital cost for the reference design reactor is $\$ 1200 / \mathrm{kW}(\mathrm{e})$.

We considered a number of possible startup scenarios for the field-reversed plasmas; however, the choice of a specific startup method for the conceptual design was deferred pending experimental demonstration of one or more of the schemes in a mirror machine. Basic to our plasma model is the assumption that, once created, the plasma can be stably maintained by injection of a neutrai-beam current sufficient to balance the particle loss rate. Two important physics assumptions for our reference case are that the particle confinement time is proportional to the ion-ion collision time and that the ratio of plasma minor radius to ion gyroradius $\left(S \equiv \mathrm{a} / \rho_{i}\right)$ is limited by stability to about 5 . The parametric analysis showed that less conservative 


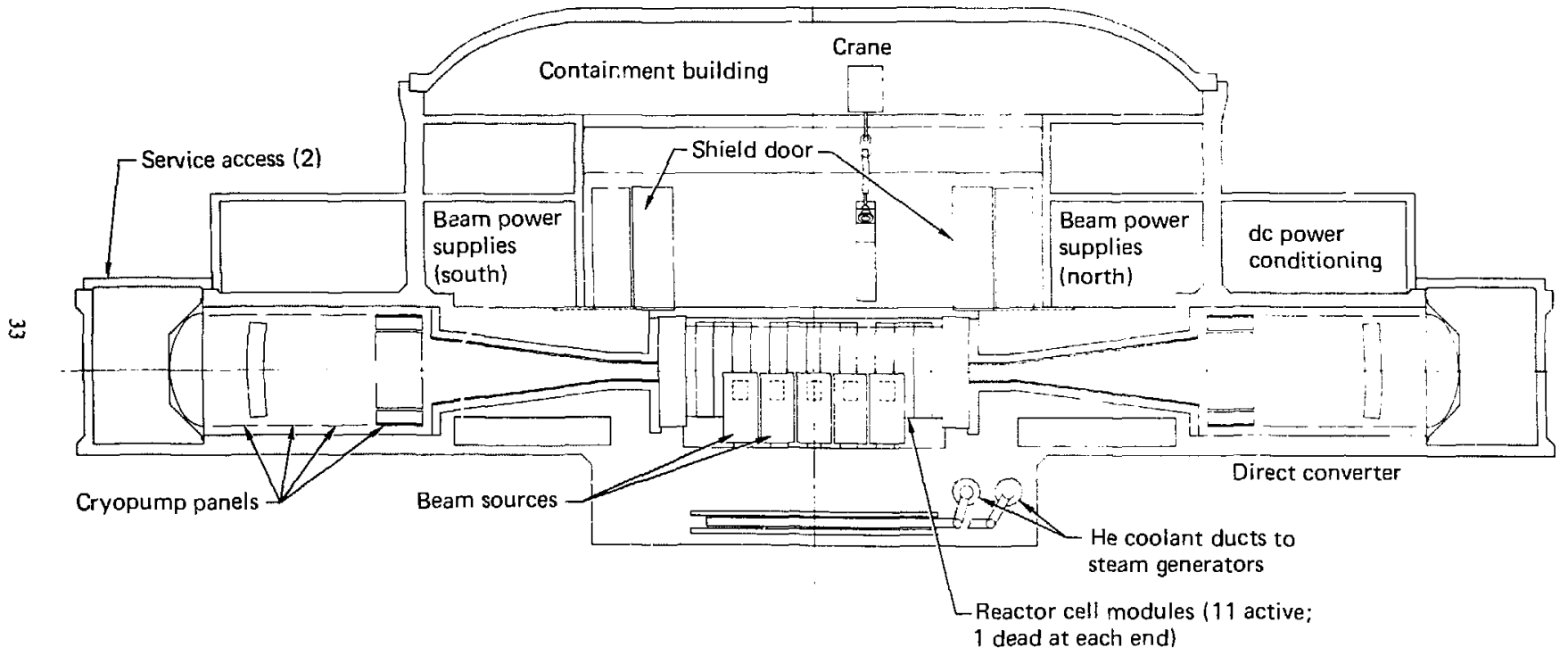

Hïg. 25. The 11-cell field-revessed mirror reactor. 
assumptions, such as classical confinement and/or $S=10$, resuit in considerably improved reactors.

The individual reactor cells for the reference design are $2 \mathrm{~m}$ long and have a cylindrical first-wall radius of about $60 \mathrm{~cm}$. The main magnetic field of $4.1 \mathrm{~T}$ is provided by $\mathrm{Nb}-\mathrm{Ti}$ superconducting solenoids with a mean radius of $3.4 \mathrm{~m}$. The plasma layers are held suparate and stable by shallow axial and radial magnetic wells produced by normal $\mathrm{Cu}$ mirror coils and loffe bars placed at the first wall. The neutral beams are injected between sections of the main solenoidal magnetic through ports in the blanket and shield. The individual reactor cells weigh about 550 tonne and can be removed for refurbishing or replacement by a gantry crane located over the reactor centerline,

The Moving-Ring Field-Reversed Mirror Reactor. (A. C. Sinith, Jr.*)

The moving-ring field-reversed mirror (FRM) reactor would produce power by burning magnetically field-reversed rings of fusion fuel that are trapped in the trough of a magnetic nirror well. This device (see Fig. 26) would use a pulsed startup mechanism (such as intense ion-beam sources, plasma guns, or relativistic electron beams) to generate an initial, field-reversed minimum-B magnetic-confinement volume filled with fuel plasma in a relatively low background magnetic field (the ring former shown in Fig. 26). A local moving mirror (provided by sequentially energizing sets of push coils located in the wall of the reactor) would then drive the plasma ring into the high magnetic field of the burner solenoid section, thereby compressing the ring and heating it to the initial burn temperature. 21 Experimental and theoretical understanding of the precise heating to be expected is somewhat sketchy, but if $C$ is the compression ratio of a characteristic linear dimension of the ring, then the plasma temperature after compression $T_{f}$ should scale from the initial temperature $T_{i}$ roughly as $T_{f} / T_{i} \approx C$ ", where $1<n<2$.

The moving-ring FRM reactor could equally serve as a startup mechanism for the steady-state FRM described in Ref. 22. The distincticn between the two concepts is in what follows compression in the moving-ring FRM reactor: whereas in the steady-state FRM reactor the confined plasma ring would be located in a fixed magnetic well and injected with high-energy neutral beams for refueling and sustenance of the diamagnetic currents, in the moving-ring FRM reactor there would be little additional energy invested in the plasma; instead, the burning plasma ring would be transported (using

\footnotetext{
"LLL Parlicipating Guest employed by the Pacific Gas and Flectric Company.
}

push coils) down the solenoidal burner section of the reactor. During its transit of the burner section, the ring could be refueled with cold plasma (either as pellets or via low-energy streaming-plasnua guns); moreover, moving the ring down the axis of the reactor would clear the way for the next ring in the procession to enter the throat of the burner. Coldion refueling allows the power produced by the plasma ring to be held constant-or varied, as the case may be- by controlling the ion temperatures and densities. Without refueling by energetic beams. the field-reversed confinement-in the absence of anomalous effects-will be sustained by the selfinductance of the ring. For fusion rings of interest, this time can easily be of the order of tens of seconds. Therefore, diffusive particle losses will determine the overall ring lifetime. The moving rings could be radially centered in the burner by either wall eddy currents or by a quadrupole field.

Depending on the fuel composition and purpose of the reactor, heat could be extracted in the conventional manner from the shield and blanket. When the plasma burn is nearly quenched (because of the cold-ion refueling), the plasma rings may be exhausted out the expande $\bar{i}$ end of the device (Fig. 26) into a direct converter. Although plasmaburns in the moving-ring FRM reactor are indeed transient events, the device could be designed to permit a fairly aniform wall heat load, as though the plasma energy source were stationary and the burn steady state.

Preliminary calculations indicate that initial burner-section plasma temperatures of 50 to 100 $\mathrm{keV}$ will be required (depending on the particle diffusion loss rates). The total radiated power per ring can probably be maintained at about $20 \mathrm{MW}$, with an overall plasma energy gain $Q \approx 5$ to 8 and ring lifetimes of about $1 \mathrm{~s}$ for rings confined in a burner with a 0.8-T magnetic field.

Tritium Control and Handling.

Tritium Processing and Control Using Active Metal Getters. (M. F. Singleton)

We are evaluating a commercially available getter pump for the Tokamak Fusion Test Reactor (TFTR). In the current TFTR design, these pumps will be used to collect the deuterium and tritium pumped from the torus between each 5-min pulse. The deuterium and tritium will then be recovered off-site from the pump cartridges.

The getter pump being evaluated for the TFTR contains an intermetaliic compound of $84 \mathrm{wt} \% \mathrm{Zr}$ $16 \mathrm{wt} \% \mathrm{Al}$ that has been powdered and pressed onto an iron substrate in a proprietary process. A photomicrograph (Fig. 27) of the powder on a substrate reveals a very thin $(\sim 2-\mu \mathrm{m})$ phase at the interface. More metallographic work on this material is 


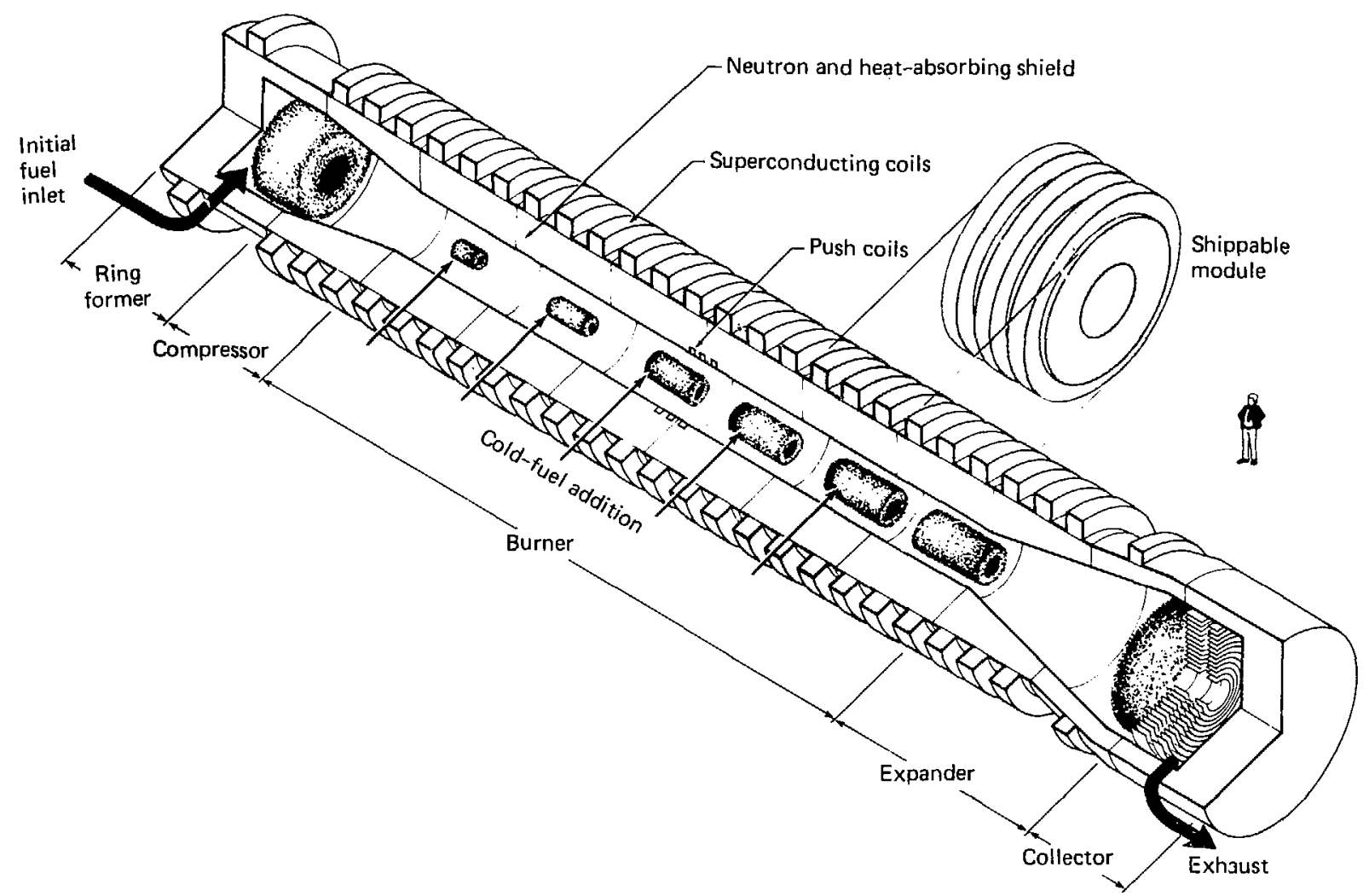

Fig. 26. The moving-ring field-reversed mirror reactor. (Illustration reproduced with the permission of PG\&E.) 


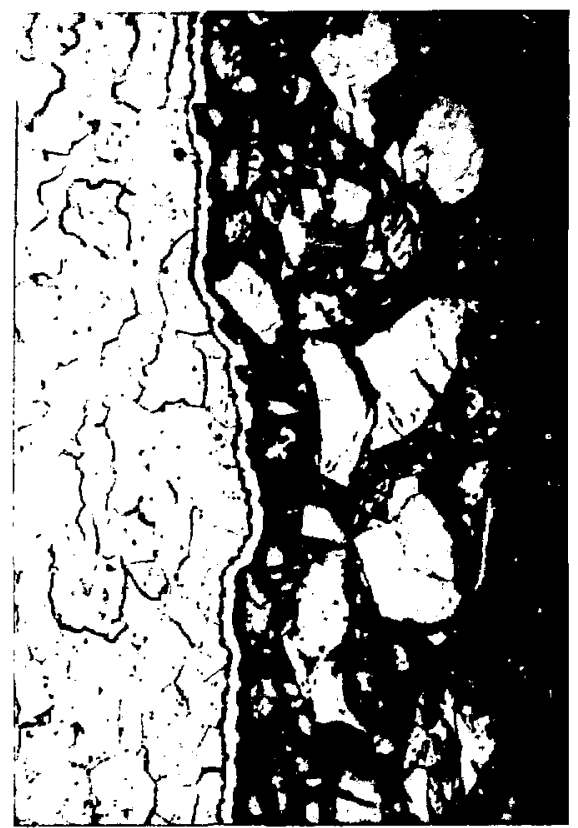

$500 \times$

Fig. 27. Photomicrograph of $84 w \% / r-16 w \%$ al powder pressed onto substrate material used in getter pump.

now in progress. Pellets of pressed bulk material have been prepared for hydriding followed by metallographic analysis.

The thin plates of $\mathrm{Zr}-\mathrm{Al}$ are arranged so as to opimize the surface-to-volume ratio and the pumping speed. The result is a circular cartridge of concentrically arranged plates placed at $4^{\circ}$ angles to each other (Fig. 28). This cartridge is mounted on a rodshaped heating element that is used to activate the $\mathrm{Zr}-\mathrm{Al}$ at $750^{\circ} \mathrm{C}$ and to keep it at $400^{\circ} \mathrm{C}$ during operation. A water jacket around the outside is to shield personnel from heat and is not necessary for proper pump performance. The pump is open to the system at only one end, and it must be evacuated from this end during the activation proces:. When the pump is in service, it acts as a dead-end trap.

Our evaluation will include a comparison between the pumping speeds obtained with each hydrogen isotope: hydrogen, deuterium, and tritium. The effect of low-level impurities (helium, nitrogen, carbon dioxide, argon, and methane) on the hydrogen pumping speed will also be assessed.
These getter pumps have fairly high pumping speeds for the active gases, but their capacity is fairly limiled. Many of these impurities cause a decrease in hydrogen pumping speed, and some may inhibit it completely. The radiolysis effects due to beta decay of the tritium may effect either pumping speed or the integrity of the getter material.

The experimental system has recently been completed, and our preliminary results on the pumping speed with deuterium are in excellent agreement with those of the manufacturer. However, we had a number of problems with heater failure when we first activated the pumps, and we feel the design will need modification for service with tritium. At present, we must open the pumps and remove the cartridge in order to repair or replace the heater. This could pose a hay, $d$ to the operator if tritium has been in the pumr.

Tritium Pricessing and Control Systems. f,A. E Sherwood!

Proposed fusion reactors will have a large tritium inventory. Containment and cleanup systems will be needed to limit personnel exposures and population dose. A Iso, fusion research facilities presently being designed will require tritium cleanup systems. Tritium can be removed from effluent air by catalytic oxidation followed by water adsorption. However, available performance characteristics and basic data for the design of tritium/air cleanup systems are inadequate. Our program has the following objectives:

- To develop mathematical models for the engineering design of tritium cleanup systems,

- To obtain data on the kinetics of oxidation and adsorption bed dynamics, and

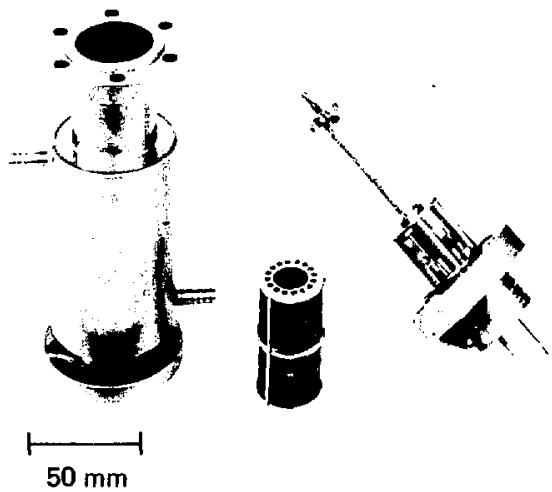

Fig. 28. Getter pump, showing (left to right) water jacket, cartridge, and heater with flange. 
- To develop the instrumentation for real-time discrimination of tritium gas and tritiated water vapor.

In March, we successfully completed the first catalyst-evaluation experiments for the TFTR in our redesigned maintenance enclosure.

Our first successful experiments on the evaluation of low-temperature catalysts for the TFTR were performed during the last week of March. The experiments were carried out in the redesigned maintenance enclosure using a $\mathrm{Pd} / \mathrm{kaolin}$ clay catalyst.

The experimental setup is shown in Fig. 29. Note that ion chamber 1 measures tritium as gas and water, while ion chamber 2 measures tritium gas only (we assume that the intermediate adsorbent bed catches all the water). Gas/water discrimination was confirmed with our first tritium run: we introduced a small amount of tritiated water into the enclosure: chamber 1 registered $\sim 500 \mu \mathrm{Ci} / \mathrm{m}^{3}$, and chamber 2 registered nearly zero $\mu \mathrm{Ci} / \mathrm{m}^{3}$. The tritiated water had been picked up, as expected, by the main adsorbent bed during the ovirnight run.

For the next run, we introduced a pulse of tritium gas into the enclostic (at $\sim 20,000 \mu \mathrm{Ci} / \mathrm{m}^{3}$ concentration) and started circulation through the cleanup system. The catalyst, loaded in the as-received condition, did not convert any tritium at all (to three significant figures) in a several-hour period.

Before the next run, we activated a batch of catalyst pellets for about $4 \mathrm{~h}$ at $400^{\circ} \mathrm{C}$ in a hydrogen gas flow and then allowed overnight cooling in a continuing hydrogen-flow stream. The activated catalyst was then loaded into the cleanup system, the pump was turned on, and a pulse of tritium gas was released into the enclosure. Ion-chamber readings for the 10 h duration of this run are shown in Fig. 30. The rapid changes in the first $10 \mathrm{~min}$ are caused, we believe, by a lack of uniform initial mixing. There is a gradual change in slope from 10 to 100 minutes caused in part, by a slight increase in air temperature: The air started at $23.0^{\circ} \mathrm{C}$, rose to $25.0^{\circ} \mathrm{C}$ at $200 \mathrm{~min}$, and reached $25.1^{\circ} \mathrm{C}$ at $400 \mathrm{~min}$.

The catalyst gave almost constant conversion from 100 to $400 \mathrm{~min}$. From the slope of the curve, given the flow rate ( $146 \mathrm{litr} / \mathrm{min}$ ) and the enclosure volume ( 5450 litre), we calculate $65 \%$ conversion per pass. From this, we compute an overall pseudohomogeneous first-order rate constant of $1.7 \mathrm{~s}^{-1}$ based on the above flow rate and a catalyst volume of 1.53 litre.

Note in Fig. 31 that ion chamber 1 shows a tailing effect starting about $100 \mathrm{~min}$ before chamber 2 . This is consistent with the presence of a small amount of tritjated water. Eventually, chamber 2 starts to tail off also, but we are then close to instrument background and accuracy is low.

In summary:

- The apparatus in the maintenance enclosure is operational, leak-tight, and appears to be working well,

- The catalyst requires activation for tritium use, and

- Reasonable cuataly tic conversion was achieved for about $8 \mathrm{~h}$ at $25^{\circ} \mathrm{C}$ and low tritium levels $\left(10^{2}\right.$ to $\left.10^{4} \mu \mathrm{Ci} / \mathrm{m}^{3}\right)$ in air at ambient humidity.

Instrument loop

Main cleanup loop

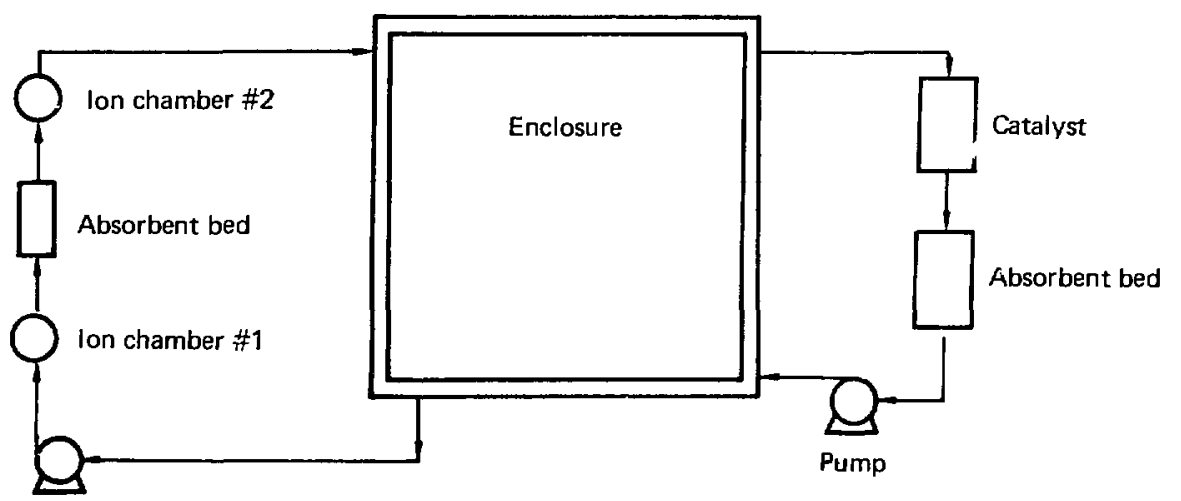

Pump

Fig 29. Experimental tritium-muintuinance enclosure system for the TFTR. 


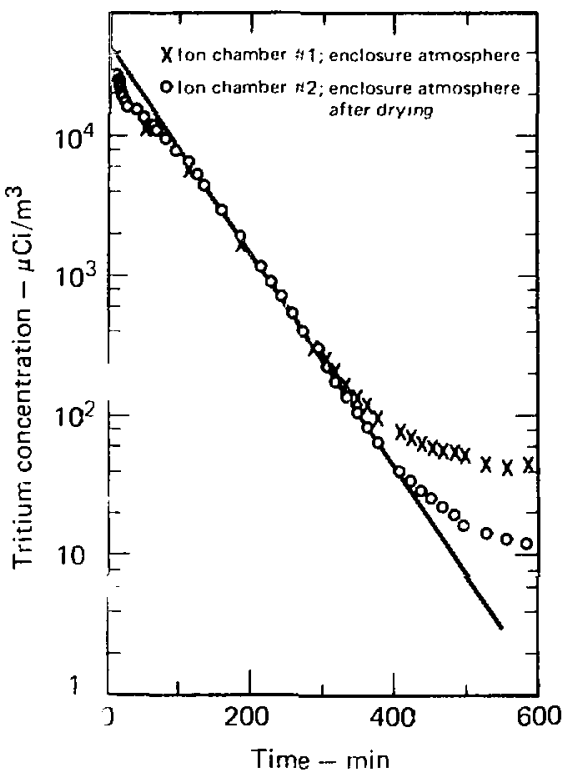

Fig. 30. ritium conversion by catalytic oxidation at $25^{\circ} \mathrm{C}$.

Croyg! nic Properties of 0-T. (P. (. Souers)

Work continuing on the compendium Cryogenic Hydroges. Properties Pertinent to Magnetic Fusion Energy. I he main structure of the document is now complete.

The compendium Cryogenic Hydrogen Properties Pertiment to Magnetic Fusion Energy is intended to gather it o a convenient reference book all pertinent dati on the hydrogen isotopes of interest to fusion technology. Thirteen new sections have been completed:

- Tritiurn Radioactivity

- Th Melting Curve

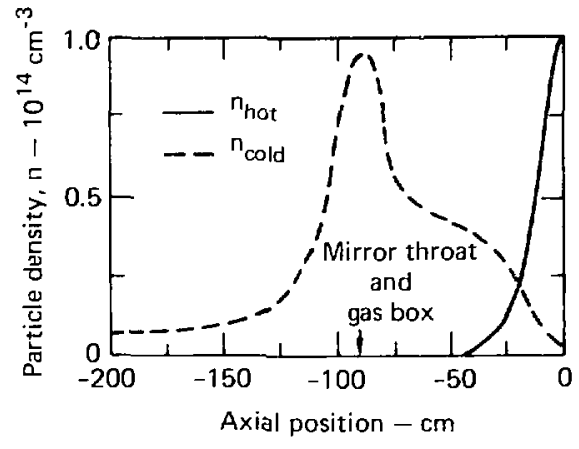

Fig. 31. Hot-and cold-ion density profiles in 2 XJIB calculated for 5 , (W) $A$ of gas current by the PHI. (NW code for use in the bIonte ('arlo calculation.

- The Liquid under Low Pressure

- The Solid Under Low Pressure

- Liquid Thermal Conductivity

- Quadrupole Energy and Heat Capacity

- Sound Velocity in the Solid and Liquid

- Dielectic Constant of the Liquid and Solid

- Debye Temperatures

- Solid Self-Diffusion and Quantum Corresponding States

- Zero-Pressure Kinetic Gas Data to $30 \mathrm{~K}$

- Nuclear Magnetic Resonances

- Hydrogen-Helium Mixtures

The early section titled "Saturated Vapor Pressures" was redone to provide consistent equations, for the first time, across all liquid and solid isotopes. Also, we have made a good start on determining the low-pressure equation of state (EOS) for $\mathrm{H}_{2}$ with direct application to the liquid jet program at Physics International in San Leandro. CA. A first, simple, EOS model has been developed that shows a very narrow window for adiabats in compressing nuid $\mathrm{H}_{2}$. 


\section{APPLIED PLASMa PHYSICS}

Applied Plasma Physics is a major sub-organizational unit of the MFE Program. It includes Fusion Plasma Theory and Experimental Plasma Research.

The Fusion Plasma Theory group has the responsibility for developing tineoreticai-computational models in the general areas of plasma properties, equilibrium, stability, transport, and atomic physics. This group has responsibility for giving guidance to the mirror experimental program. There is a formal division of the grcup into theory and computational; however, in this report the efforts of the two areas are not separated since many projects have contributions from members of both.

Under the Experimental Plasma Research Program, we are developing the intense, pulsed neutral-beam source (IPINS) for the generation of a reversed-field configuration on $2 X$ IIB. We are also studying the feasibility of utilizing certain neutron-detection techniques as plasma diagnostics in the next generation of thermonuclear experiments.

\section{Fusion Plasma Theory}

\section{Time-Dependent Tandem-Mirror Confinement Studies \\ (R. H. Cohen)}

We have used the TAMRAC code to study startup conditions in the Tandem Mirror Experiment and alpha-particle buildup in a tandem mirror reactor.

The tandem-mirror rate code TAMRAC integrates rate equations for number and energy of an arbitrary number of species in a tandem-mirror machine. In addition to sources and end loss, 23.24 the code includes models for enhancement of plug loss Jue to the drift-cyclotron loss-cone (DCLC) mode and for externally supplied streaming plasma to stabilize the DCLC mode, supplemental electron heating, species-dependent radial diffusion, and provision for injecting hot (not electrostatically confined) ions into the solenoid. We have used TAMRAC for the following two studies:

- A study of startup in the Tandem Mirror Experiment (TMX) that indicates that near steadystate conditions can be achieved on a time scale allowed by the power supplies if the gas feed is suitably programmed, and

- A study of alpha-particle buildup in a tandem mirror reactor, and ways of preventing buildup to a level that seriously degrades reactor $Q$. One particular means of eliminating hot alpha particles, nonadiabatic scattering (magnetic moment stochasticity), has been studied in detail.

Superadiabatic Ion Motion in the Presence of an Electrostatic Wave in a Mirror Machine (G. R. Smith, J. A. Byers, and L. L. Lodestro)

Studies of ion motion in an axisymmetric magneticmirror field show that superadigbaticity affects iony with perpendicular energy greater that $8 \mathrm{keV}$.
Rosenbluth ${ }^{25}$ and Tinnofeev ${ }^{26}$ showed that a monochromatic flute mode at the ion-cyclotson frequency causes ions with low perpendicular energy $W_{1}$ to move stochastically, while ions with high $W_{\perp}$ move superadiabatically we have improved their model by including the azimuthal $\nabla$ B-drift. Also, we used Rosenbluth's method and developed a new, more general method for determining the boundary in velocity space between stochastic and superadiabatic motion. The new method is based on the overlap of the bounce resonances defined by $\omega$ $k v_{D}=!-2 n \omega_{b}$, where $\omega-k v_{D}$ is the wave frequency, Doppler-shifted by the $\nabla$ B-drift, $\omega_{h}$ is the frequency of bouncing between mirrors, $\bar{\Omega}$ is the bounce-averaged cyclotron frequency, and $n$ is any integer. Both theoretical methods predict that ions with $W_{\perp}>W_{L S} \approx 8 \mathrm{keV}$ move superadiabatically: this result agrees with the behavior of calculated ion orbits in a model magnetic field. The value of $W_{\perp s}$ depends only weakly on the axial and radial scale lengths of the magnetic field and on the amplitude $\phi$ of the flute mode $\left(W_{\perp,} \propto \phi^{0.21}\right)$. Such a low value for $W_{\perp 5}$ disagrees with experimental results that. apparently, indicate stochastic motion of ions with $W_{\perp}=40 \mathrm{keV} .{ }^{27}$ This discrepancy cannot be resolved, we believe, either by including the collisional drag by electrons, or by replacing the present slab model by a cylindrical one. However. inclusion of effects of the quadrupole field may significantly alter the above results.

\section{Simple Rotating-Field Reversed-Plasma Equilibria \\ (B. McNamara)}

We have examined models of rotating equilibria that will be useful in studying the stability of the reversed-field theta pinch.

Wc have surveyed rotating cquilibria, such as are generated in the Los Alamos Scientific Laboratory 
(LASL) reversed-fiteld theta pinch, as a function of five parameters: plasma beta, pressure profile, rotation velocity, aspect ratio of the theta pinch, and the mirror ratio of the vacuum field. Several models have been examined, the simplest of which is the one-fluid, scalar-pressure, rigid-rotator model. The equilibrium is described by two equations: a Bernoulli-type relation between the density $o$ and the electric potential $\phi(\psi)$,

$$
\mathrm{B} \cdot \nabla\left[\mathrm{C}_{\mathrm{s}}^{2} \ln \rho-\frac{\mathrm{r}^{2}}{2}\left(\frac{\partial \phi}{\partial \psi}\right)^{2}\right]=0,
$$

where $C_{S}$ is the speed of sound: and the radial pressure balance equation for the poloidal magnetic flux $\psi$.

$$
\Delta^{*} \psi+4 \pi \mathrm{C}_{\mathrm{s}}^{2} \mathrm{r}^{2} \partial \rho\left(\psi, \mathrm{r}^{2}\right) / \partial \psi=0
$$

These equilibria represent a starting point for magnetohydrodynamic (MHD) stability studies of the reversed-field theta pinch and for the more complicated two-dimensional equilibria in tandemmirror devices.

\section{Rotational Instabifities in Reversed-Field Configurations \\ (L. D. Pearlstein and L. Lodestra)}

Our calculations of rotational instabilities in reversed-field theta pinches agice with ohserved mode frequencies but not with rotational velocities.

Reversed-field theta pinches disrupt because of an $m=2$ rotational instability. In these configurations, $L$ (the plasma length) $\gg R$ (the plasma radius); thus, $B_{z} \gg D_{r}$ except where the field lines turn. Equilibrium satisfies the "long-thin" approximation, $2 p_{\perp}+B^{2}=B_{v a c}^{2}$, and $B=B_{z}$ almost everywhere. Since rotational modes are flutes, we average over closed field lines, neglecting contributions from where the field lines turn. This produces the differential equation (for rigid rotation at frequency $\Omega$ )

$$
\begin{aligned}
\frac{d}{d \eta}\left(\eta^{2}+\eta_{0}^{2}\right) \ell(n) \rho T \frac{d \psi}{d n} \\
\quad-\left[\frac{m^{2}-1}{4} \rho T+2 \omega^{2} \eta \frac{\partial \rho}{\partial \eta}\right] \ell(\eta) \psi=0 .
\end{aligned}
$$

Here, $\eta$ is a function of the flux such that $B^{2}=B_{v}^{2}$ $\tanh ^{2} \eta$ and $\eta_{0}$ is the value of $\eta$ at the last closed field line, $\ell(\eta)$ is the length of a field line, and

$$
\begin{aligned}
\rho \mathrm{T}=(\omega-\Omega) & {[\omega-\Omega} \\
& \left.+\omega_{*}\left(1+\frac{1}{2 \sinh ^{2} \eta}\right)\right] \frac{1}{\cosh ^{2} \eta} .
\end{aligned}
$$

The singularity at $r=0$ disappears because of averaging. Note that $(\eta=0)$ now becomes a boundary point where $E_{0}=0$ (perfect conduction), but $\psi$ $=E_{j}^{\prime} / B$ is indeterminate. To resolve this ambiguity, one can include a $B_{b}$ in equilibrium that suggests $\psi$ $=0$. Alternatively, one $c$. In solve the boundarylayer problem where the FLR expansion is not valid.

To date, we have only compared stability boundaries from a variational procedure and a shooting procedure with the boundary conditions $\psi^{(0)}=0$. Results for $\psi=0$ indicate that the frequency of the mode agrees with that observed. However, instability is predicted to occur at a rotational velocity considerably in excess of that inferred experime.]tally from Doppler-shifted impurity radiation.

\section{Orbital Resonances in Quadrupole-Stabilized Mirror Conśigurations}

(R. H. Cohen, D. V. Anderson, and

C. Sharp)

Calculations suggest that the orbital resonances manifested as sudden losses of plasma ("bursts") in RECE-Berta should be considerably smaller in $2 \mathrm{X}$ IIB.

Using analytic techniques and the particle-orbit code ORBXYZ, we have demonstrated that a quadrupole field added to an axisymmetric, nonvacuum mirror configuration significantly couples the radial, azimuthal, and axial motions of single particles, and that this effect may account for the bursting and nonbursting anomalous losses observed in the relativistic electron compression experiment (RECE-Berta) at Cornell. ${ }^{28}$ At particular values of the plasma self-field, a class of resonant particles can experience sizable excursions from an initial set of radial and axial oscillation amplitudes. If the plasma radius is defined by a limiter, as in RECE-Berta, then resonant particles hitting the limiter would give rise to a sudden loss of plasrna. From our analytic estimates of the resonance conditions and resonance widths, we have estimated the size of a burst. Orbit-code calculations in steadystate and time-varying fields are used to show the effect. Between bursts. resonances mainly affect particles away from the limiter, giving rise to an enhancement of classical diffusion, such as is also observed in RECE-Berta. In 2XIIB, resonance effects 
should be considerably smaller at a given fraction of field reversal, and should not be manifested as bursts.

Spatial Eigenmodes for the Alfvén

Ion-Cyclotron Instability in

Finice-Length Plasma

\section{(D. C. Watson and T. D. Rognlien)}

Calculations of spatial eigenmodes and stability limits based on a finite-length plasma agree with earlier deductions and calculations.

We have investigated improvement in Alfvén ioncyclotron (AIC) stability brought about by finitelength plasma and we have also examincd the eigenmode (E-field) structure. For ease of investigation, we chose a finite-length plasma model where ions are confined by a parabolic electrostatic potential well and lie in a uniform magnetic field. The electrons maintain quasi-neutrality. The MaxivellVlasov system was solved in full, including the ion bounce motion.

First, we derived heuristic dispersion relations; second, we calculated exact stability limits; third, we displayed the important eigenmodes. We found that modes with spatially even E-fields have resonances at $\omega=\omega_{\mathrm{ci}} \pm 2 n \omega_{\mathrm{bi}}$, whereas modes with spatially odd E-fields have resonances at $\omega=\omega_{\mathrm{ci}} \pm(2 \mathrm{n}-1)$ $\omega_{b i}$. Here, $\omega_{c i}$ and $\omega_{b i}$ are the ion cyclotron and bounce frequencies, respectively.

The lowest-order odd mode is the most unstable. The onset of instability roughly agrees with that deduced from the infinite-medium dispersion relatios and the requirement that a half-wavelength should fit in the plasma. These results are in accord with those from Byers' linearized particlesimulation code, which uses the same finite-plasma model. Byers' code ${ }^{29}$ has a linear-polarization constraint that introduces additional resonances with $\omega_{\mathrm{ci}} \rightarrow-\omega_{\mathrm{cj}}$ in the equations above. This leads to the complication of double resonances when $\omega_{\mathrm{bi}}$ $=\omega_{\mathrm{ci}} / \mathrm{n}$.

\section{Electron Effects in Ion-Current}

Field Reversal

\section{(D. E. Baldwin and M. E. Rensink)}

Using a s mple model, we have datermined situations in whicti significant electron currents develop in an axisymmetric plasma composed of more than one ion species.

Existing calculations of field reversal driven by ion currents ha: e neglected all electron effects other than ion energy loss. These have assumed that the generation of a current by ionization of neutralbeam atoms results from the charge separation induced by the magnetic field; all plasma currents are carried by the ions. However, electron currents can be generated by two means:

- Electron-ion momentum transfer tends to equalize the average velocities of these particles, partially cancelling the ion current. This mechanism is particularly effective at a field null, and

- Radial electric fields are set up by the charge separation that results from ionization or unequal transport rates. In these fields, electrons can drift in a way that cancels the ion current.

Using a simple model of electron-ion momentum transfer, we have calculated electron currents in the presence of assumed ion species. We find the following general conclusions for an axisymmetric configuration:

- The existence of a field null in steady state requires a net current at the null. This can arise in a plasma composed of more than one ion species where the ions have different charges and average velocities. Most simply, a nonı otating species offers a resistive background so that the electrons cannot accelerate to nullify the current of a rotating ion species.

- Away from a field null, an azimuthal electron drift velocity arises only because of a radial electric field.

- On open magnetic lines, electron endconduction to grounded boundaries constrains the variation in potential to the order $\mathrm{T}_{\mathrm{e}} / \mathrm{e}$. A zimuthal electron drift currents are small compared with the ion current and are jusiifiably neglected.

- On closed magnetic lines, large potentials can develop between magnetic flux surfaces, and the possibility of significant cancellation of the ion current by drifting electrons exists.

A quantitative assessment of these effects requires a prescription for calculating electric fields and electron currents in ion-particle codes such as SUPERLAYER at LLL. This is being implemented.

\section{Axial Profile of Electron Temperature via Monte Carlo Electrons \\ (T. D. Rognlien)}

We have developed a Monte Carlo electron code to calculate the axial electron hegt flow in 2XIIB.

The electron temperature $T_{e}$ measured in the 2XIIB experiment shows that the central temperature can increase from $50 \mathrm{eV}$ to $150 \mathrm{eV}$ as the plasma density is increased. In contrast, the electron temperature is considerably lower in the region outside the mirror throat and gas box, with $\mathrm{T}_{\mathrm{e}}$ ranging from $20 \mathrm{eV}$ to $40 \mathrm{eV}$. The theoretical description of axial electron heat flow is complicated by the fact that the mean free path for electrons is of the same 
order as the scale length of density variation in the system. Consequently, neither a classical fluid description nor a bounce-averaged Fokker-Planck description of the electron heat flow is valid. To treat this problem properly, we have developed a Monte Carlo computer code for the electrons.

A basic outline of the Monte Carlo electron code is as follows:

- The electron orbits are followed in assumed or previcusly calculated magnetic and electric fields.

- The electrons are randomly scattered at each time step according to their Coulomb scattering coefficients.

- The average energy of such electrons is calculated at a number of axial positions and for many time-steps.

This sequence gives statistically meaningful information about the axial temperature profile. Details of the various parts of the calculation follow.

The electron orbits are followed along a magnetic field line: we assume that the magnetic moment is conserved between collisions. The electron equation of motion is thus

$$
m_{e} \frac{d^{2} z}{d^{2}}=m_{e} \frac{d v_{r}}{d t}=e \frac{\partial \Phi}{\partial z}-\mu \frac{\partial B}{\partial z},
$$

where $z$ is the axial position, $m_{e}$ is the electron mass, $\mathrm{e}$ is the electronic charge, $\Phi$ is the electrostatic potential, $B$ is the magnetic field, $\mu=m_{e} v_{\perp}^{2} / 2 B$ is the magnetic moment, and $v_{1}$ is the velocity perpendicular to $B$. The fields $\Phi(z)$ and $B(z)$ are taken from the axial fluid code PHLOW, which describes the axial flow of cold plasma from the gas box. For given hot-ion density and electron temperature profiles, the PHLOW code calculates a selfconsistent $\Phi(z), n_{e}(z)$, and $n_{c}(z)$, where $n_{e}(z)$ is the electron density and $n_{c}(z)$ is the cold-ion density.

After an electron is pushed one time step $\Delta t$ according to Eq. (1), its velocity components are changed to reflect the effect of Coulomb collisions. A velocity coordinate system is formed in which the electron velocity vector lies along the $\mathrm{w}_{\|}$axis. The electron is then scattered in this coordinate system according to

$$
\begin{aligned}
\mathrm{w}_{\|} & \rightarrow \mathrm{w}_{\|}+\left\langle\Delta \mathrm{w}_{\|}\right\rangle \Delta \mathrm{t}+\left(3\left\langle\Delta \mathrm{w}_{\|}^{2}\right\rangle \Delta \mathrm{t}\right)^{1 / 2} \mathrm{R}_{1} \\
& \mathrm{w}_{\mathrm{x}} \rightarrow\left(1.5\left(\Delta \mathrm{w}_{\uparrow}^{2}\right\rangle \Delta \mathrm{t}\right)^{1 / 2} \mathrm{R}_{2} \\
& \mathrm{w}_{\mathrm{y}} \rightarrow\left(1.5\left\langle\Delta \mathrm{w}_{1}^{2}\right\rangle \Delta \mathrm{t}\right)^{3 / 2} \mathrm{R}_{3}
\end{aligned}
$$

Hese, $R_{1,2,3}$ ase random numbers uniformly distributed from -1 to 1 . The scattering coefficients are obtained from Spitzer 30

$$
\begin{aligned}
\left\langle\Delta w_{1 j}\right\rangle=-A_{c} & {\left[2 n_{c} \ell_{c} G\left(\ell_{c} v\right)\right.} \\
& \left.+n_{h} \ell_{h} G\left(\ell_{h 1} v\right)+n_{c} \ell_{c} G\left(\ell_{c} v\right)\right],
\end{aligned}
$$

$$
\left\langle\Delta w_{H}^{2}\right\rangle=\frac{A_{c}}{v}\left\{n_{c} G\left(\ell_{c} v\right)+n_{h} G\left(\ell_{h} v\right)\right.
$$

$$
\left.+n_{c} G\left(\ell_{c} v\right)\right]
$$

$$
\begin{aligned}
\left\langle\Delta w_{1}^{2}\right\rangle=\frac{A_{c}}{v}\left[n_{c} H\left(\ell_{c} v\right)+\right. & n_{h} H\left(\ell_{1} v\right) \\
& \left.+n_{c} H\left(\ell_{c} v\right)\right],
\end{aligned}
$$

where $v=\left(v_{2}^{2}+v_{1}^{2}\right)^{1 / 2}$. Other quantities are

$$
\begin{aligned}
& G(x)=\left[\operatorname{erf}(x)-x \operatorname{er} f^{\prime}(x)\right] / 2 x^{2} \\
& H(x)=\operatorname{erf}(x)-G(x) \\
& A_{e}=8 \pi e^{4} \ln L / n_{e}^{2} \\
& f_{a}^{2}=\left(2 k T_{a} / m_{a}\right)^{-1}
\end{aligned}
$$

The subscripts $e, h$, and $c$ refer respectively to electrons, hot ions, and cold ions, and $\ln \Lambda$ is the Coulomb logarithm. The spatial profiles of particle densities $n_{e}, n_{h}$, and $n_{c}$ are taken from the PHLOW code. After the electron is scattered in the w-velocity space, its velocities are converted back to the $v_{z}$ and $v_{\perp}$ space. The process is then repeated with the electron advanced according to Eq. (1).

We now have a version of the Monte Carlo code that calculates the eleciron temperature profile for fixed profiles of $n_{e}, n_{h}, n_{e}$, and $T_{e}$. Shown in Fig. 31 is a sample of the profiles used as obtained from the PHLOW code for a high-current case of $I_{\text {gas }}$ $=5,000 \mathrm{~A}$. Recall that $n_{\mathrm{e}}=\mathrm{n}_{\mathrm{h}}+\mathrm{n}_{\mathrm{c}} ; \mathrm{n}_{\mathrm{e}}$ is therefore omitted from the figure. The $T_{e}$ profile is assumed to be $50 \mathrm{eV}$ at the left-hand wall and to rise to 100 $\mathrm{eV}$ in the center. The initial temperature profile and that of the Monte Carlo electrons is given in Fig. 32. 
The statistical fluctuation is evident. Note that the temperature does not depart greatly from the assumed $T_{e}$ profile because the electron-electron interactions are stronger than those causing hot-ion heating and cold-ion cooling. The temperature in the center of the profile is $\sim 105 \mathrm{eV}$; that at the end is $\sim 50 \mathrm{eV}$. The heating in the center arises from hot ions; the cooling at the ends is from electron strath losses. We emphasize that our results are not final, but rather that they show the information that can be obtained from the Monte Carlo code.

Our next step will be to iterate the Monte Carlo code and the PHLOW code until the $T_{e}(z)$ profiles are the same in each. This self-consistent result is the ultimate goal of the calculation.

\section{A Local Theory of Nonlinear Ion Dynamics in a Drift-Cyclotron Mode}

(B. I. Cohen. N. Maron, and

T. D. Rogntien)

Nonlinear perturbation theory and a new hybrid model (fluid electrons and particle ions) for onedimensional, fully electrostatic computer simulation have been used to study the nonlinear theory of the drift-cyclotron instability.

The interaction of the ion diamagnetic-drift wave with an ion Bernstein wave can lead to the wellknown drift-cyclotron instability. In mirror machines, the drift cyclotron mode becomes important because it can persist as a residual instability after electrostatic turbulence (resulting from the DCLC mode) and externally introduced streaming plasma have partially filled the loss cone in velocity space. ${ }^{31}$ Local theory ${ }^{32-34}$ has suggested that nonlinear ion dynamics induced by a single wave can stabilize the drift-cyclotron mode by disrupting the necessary frequency synchronization between the mode frequency $\omega$, the nearest cyclotron harmonic $N \omega_{c i}$, and the ion diamagnetic frequency $\omega^{*}$. We consider three particular regimes: near linear marginal stability $\omega \approx N \omega_{c j} \approx \omega^{*} / 2,{ }^{32}$ near the simultaneous resonance $\omega \approx \omega^{*} \approx N \omega_{\mathrm{ci}}$, ${ }^{33}$ and in the regime of ion trapping. ${ }^{34} \mathrm{We}$ have reviewed the theories of the stabilization caused by a nonlinear frequency shift 32,33 within our own analytical framework and have found significant discrepancies. We have derived a nonlinear equation of the form

$$
\left[\left(\partial^{2} / \partial t^{2}\right)-\gamma^{2}\right] \psi+\alpha \mid \psi ! 2 \psi=0
$$

where $\psi=\mathrm{e} \Phi / \mathrm{T}, \gamma=$ the linear growth rate, and $\alpha$ is a coupling coefficient associated with the nonlinear (specifically quasi-linear in origin) corrections to Poisson's equation at third order in the mode amplitude. A nonlinear dissipation shift acts as a stabilizing influence $(\alpha>0)$ on both the driftcyclotron and DCLC instabilities. There is a concomitant shift in the real part of the mode frequency at saturation, which is the order of the linear growth rate in magnitude.

To confirm theoretical predictions and further understand the role of nonlinear ion dynamics in the stabilization of drift-cyclotron and DCLC instabilities, we have developed a hybrid model (fluid electrons and particle ions) for one-dimensional, fully electrostatic computer simulation. The algorithm suffers no restriction due to electroncyclotron or plasma-frequency time scales, and is only required to accurately follow lower-hybrid and ion-cyclotron frequencies (Fig. 33). We are using

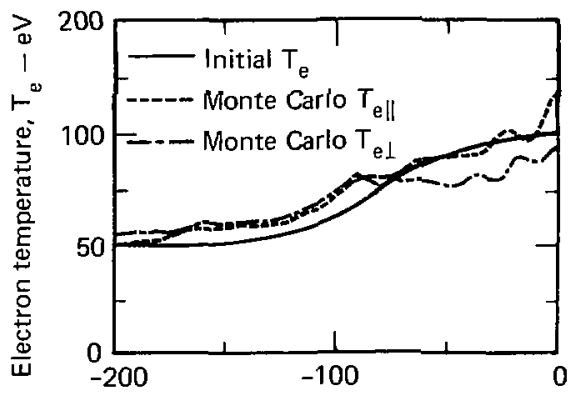

Fig. 32. Comparison of initial electron temperature and the first iteration of the Monte Carlo electron temperature.

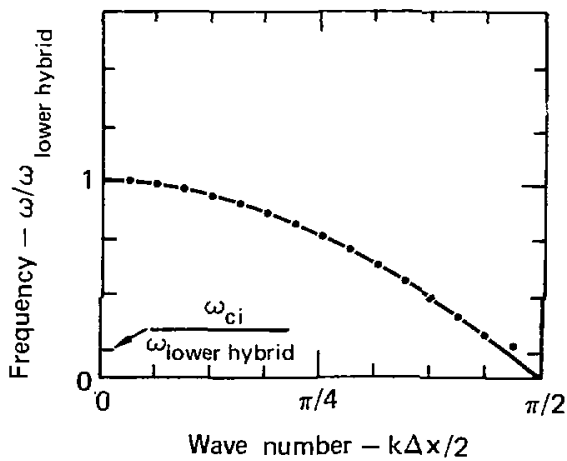

Fig. 33. Numerical dispersion properties of the one-dimensional slab, hybrid simulation code. The theoretical grid-corrected linear dispersion relation for small-amplitude lower hybrid w: es in a cold, uniform plasma is plotted, $\omega / \omega_{\mathrm{ci}}=\left(\omega_{\mathrm{b}} / \omega_{\mathrm{d}}\right)$ $[\sin (k \Delta x / 2) /(k \Delta x / 2)]^{3 / 2} \cos ^{1 / 2} k \Delta x / 2$, with data obtained from simulation superposed for comparison $\mid \omega_{\mid \mathrm{h}} \approx \omega_{\mathrm{c}} /\left(\mathrm{m}_{\mathrm{e}} / m_{\mathrm{i}}\right.$ $\left.+\omega_{\mathrm{ci}}^{2} / \omega_{\mathrm{pl}}^{2}\right)^{1 / 2}$ ]. 


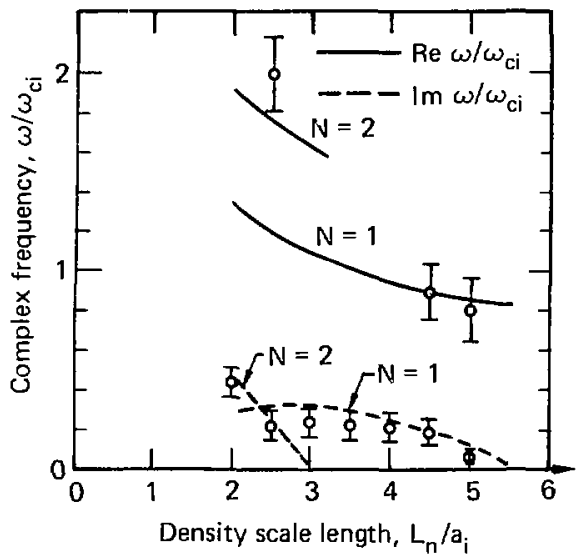

Fig. 34. Simulations of the drift-cyclotron instability confirming linear theory. The complex frequency $\omega / \omega_{\mathrm{c}}$ is plotted as a function of scale length $\mathrm{L}_{n} / \mathrm{a}_{i}$ for $\mathrm{N}=1,2$ where $\operatorname{Re} \omega \approx N \omega_{\mathrm{c}}$ and $k a_{i}=7$, $\omega_{p i}^{2} / \omega_{c i}^{2}=m_{i} / m_{e}=100$, and $\omega_{r i} \Delta t=0.05$. Increasing turbulence occurs as $L_{n} / a_{i}$ decreases. The real part of the frequency has not been messured in simulations where there is an eariy onset of nonlinear behavior.

the simulations to study the stability of systems that could be stabilized by a complex frequency shift in the regime of weak instability where our analytical theory is expected to be valid. In regimes of strong instability we expect ion trapping. So far, our simulations have confirmed the linear theory of the drift-cyclotron instability (Fig. 34) and have evidenced ion trapping in the nonlinear regime. When the electric field amplitude is sufficiently weak so that trapping does not occur, simulations of a single unstable mode confirm our theoretical description of saturation by means of a nonlinear frequency shift. However, preliminary multi-mode simulation results suggest that this saturation mechanism may fail in the presence of other modes (Fig. 35).

\section{Experimental Plasma Research}

The Intense, Pulsed Ion-Neutral

Source (IPINS)

(D. S. Prono)

Our principal goal is to develop intense, pulsed, neutral-beam sources (IPINS) for the mirror program. The generation of a reversed-field configuration in a 2 XII B-scale experiment with rapid (microsecond time scale) buildup times would be the main near-term application of this technology, and the current research is focused on the achievement of the performance levels required by this application.

The major test stand is now operational. Our experiments have emphasized

- Diagnostic fabrication, installation, and debugging;

- Initial studies of beam divergence; and

- Studies of how geometrical changes in the diode affect the rapid anode-catnode closure observed in this system.

Because the data are preliminary, we shall not attempt a detailed discussion of our results to date. Briefly, this system has exhibited the low-impedance ion-flow mode with the diode voltage remarkably constant (as the current changes and the gap closes) and with the voltage level controlled by the properties of the anode foil. This behavior is, of course, in good accord with the simple one-dimensional steady-state theory. The major problem is the short duration of the ion-flow mode, which is terminated by diode closure after a much shorter time (closure velocities $>10 \mathrm{~cm} / \mu \mathrm{s}$ ) than with the small test stand. Although we do not understand the origin of this rapid anode-cathode closure, we have demonstrated how the inherent "twodimensionality" of the diode geometry of the small test stand (asymmetric current in the foil and a small $B_{2}$ field) lessens the closure speed there. For a number of reasons, operation in this fashion may not be desirable, and we are therefore attempting to extend the ion-flow duration by expanding the anode-cathode gap.

We are designing a modification to the diode geometry that will allow larger anode-cathode spacings, improved access to and visibility of the diode region, and a revised vacuum insulator support structure. The last should alleviate the flashover difficulties that occur with a longer ion-flow pulse length. The modified design should be installed early in the next quarter. 


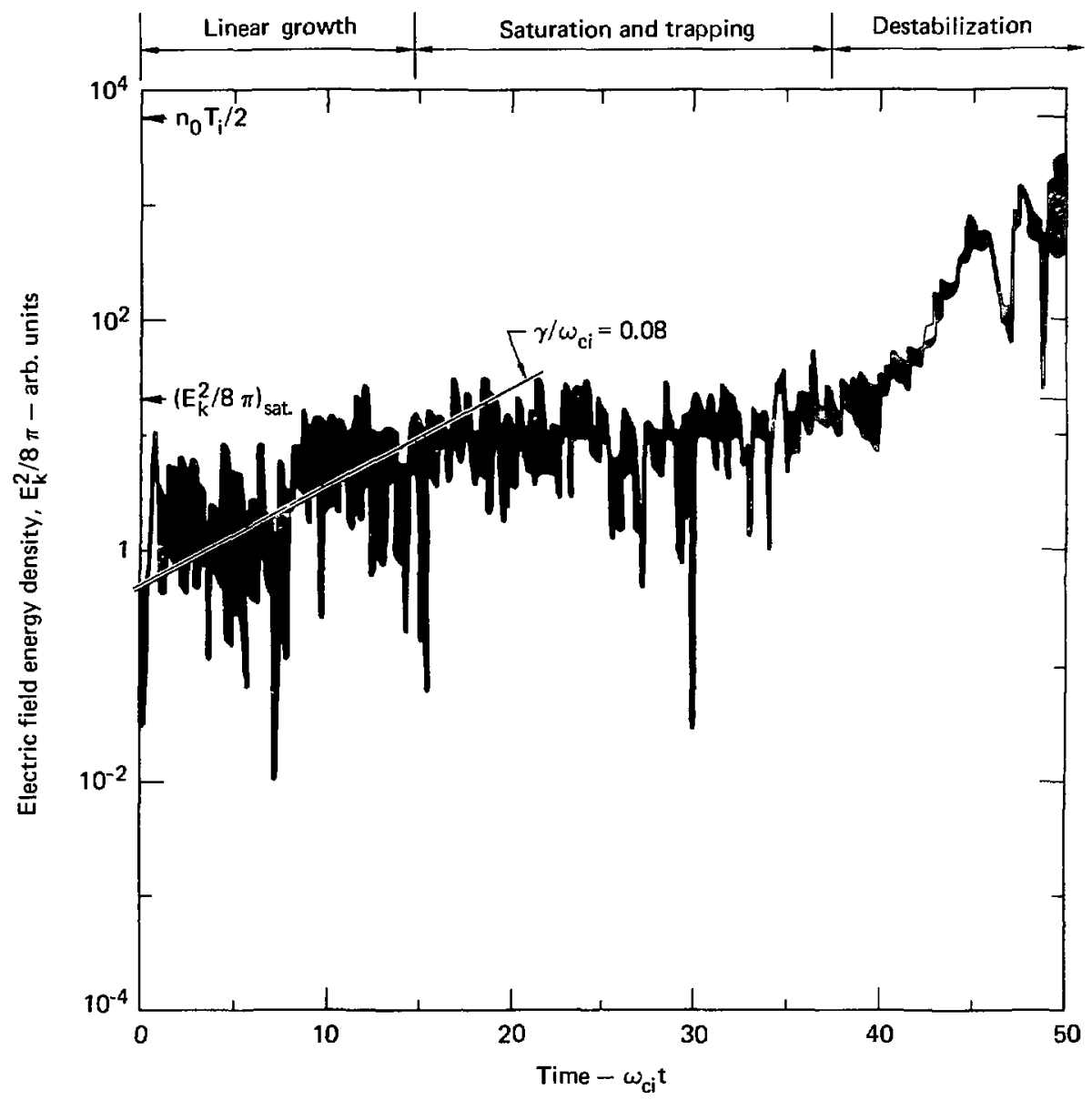

Fig. 35. Nonlinear evolution of a drift-cyciotron mode in the presence of other modes. Plotted here is the electric field energy density for a single mode $k a_{i}=7$ as a function of time for $\omega_{p i}^{2} / \omega_{c i}^{2}=m_{i} / m_{c}=100$, $L_{n} / R_{1}=4.9$, and $\omega_{c i} \Delta t=0.05$. There are other lineary unstable modes present that apparently destablize the single mode shown subsequent to its soturation at $\omega_{d} t \approx 20$ with an amplitude $\left(E_{k}^{2} / 8 \pi\right)_{s a 1}$, in fairly good agreement with our nonlinear perturbation theory. The instability late in the simulation is preceded and accompanied by considerable ion trapping. 


\section{REFERENCES}

1. M. A. Harrison, Scientific Editor, Magnetic Fusion Energy Quarterly Report-October through Decenter 1977. Lawrence Livermore Laboratory, Rept. UCRL-50051-77-4 (1978).

2. W. C. Turner, E. J. Powers, and T. C. Simonen, "Properties of Electrostatic Ion-Cyclotron Waves in a Mirror Machine," Phys. Rev. Lett. 39, 1087 (1977).

3. A. W. Molvik, "Measurements of Stabilizing Warm Plasma," Bull. Am. Phys. Soc. 22, 1143 (1976).

4. M. A. Harrison, Scientific Editor, Magnetic Fusion Energy Annual Report-Octoher 1976 through Seplember 1977, Lawrence Livermore Laboratory, Rept. UCRL-50002-77 (in preparation).

5. T. D. Rognlien, Lawrence Livermore Laboratory, private communication (1978).

6. J. P. Holdren, Analytical Approximation to Collisional Distributions in Mirror Plasma, Lawrence Livermore Laboratory, Rept. UCRL-72391 (1978).

7. T. B. Kaiser, Lawrence Livermore Laboratory, Internal Document MFE/TC/77-104 (1977). Readers outside the Laboratory who desire further information on LLL internal documents should address their inquiries to the Technical Information Department, Lawrence Livermore Laboratory. Livermore. California 94550.

8. R. E. Klinkowstein and L. D. Smullin, "Suppression of $\omega_{\text {ci }}$ Instability in a Mirror-Confined Plasma by Injection of an Electron Beam." Phys. Rev. Lett. 40, 771 (1978).

9. E. S. Ritter, W. C. Rutledge, and R. H. Ahlert, J. Appl. Phys. 28, 1468 (1957).

10. F. H. Coensgen, Project Leader, TMX Major Projed Proposal, Lawrence Livermore Laboratory, Rept. LLL-Prop-148 (1977).

11. I. H. Foote, Allowable Misalignment of Various Elements of the TMX Magnet Set. Lawrence Livermore Laboratory, Rept. UCID-17766 (1978).

12. F. H. Coensgen, Project Leader, MX Major Project Proposal, L.awrence Livermore Laboratory, Rept LLL-Prop-142 (1976). [Note: The MX designation was later changed to Mirror Fusion Test Facility (MFTF).]

13. D. W. Deis, D. N. Cornish, D. G. Hirzel, A. R. Rosdahl "Strain-Critical Current Data for Large Multifilament $\mathrm{Nb}_{3} \mathrm{Sn}$ Conductors" in Proc. $7^{\text {th }}$ Symp. Eng. Problems of Fusion Research. Knoxville. 1977 (IEEE. 1977).

14. D. Dreyfuss, B. Augenstein, W. Mooz, and P. Sher, An Examination of Alternate Nuclear Breeding Methods, Rand Corporation, Rept. R-2267-ERDA (in preparation).

15. H. A. Bethe, The Fusion Hybrid. Lawrence Livermore Laboratory, Rept. (in preparation).

16. F. Fortesque, "Comparative Breeding Characteristics of Fusion and Fast Reactors," Science 196, 1326 (1977).

17. D. I. Bender, Performance Parameters for Fusion-Fission Power Systems, Lawrence Livermore Laboratory, Rept. UCRL-80589, Rev. 1 (1978).

18. "Mirror Fusion-Fission Reactor Designs," in Proc. US-USSR Symp. Furion-Fission Reactors, Livermore. 1976 (CONF-760733, 1976), p. 13.

19. D. J. Bender, J. D. Lee, W. S. Neef, R. S. Devoto, T. R. Galloway, J. H. Fink, K. R. Schultz, D. Culver, R. Rao, and S. Rao, "The Mirror Hybrid (Fusion-Fission) Reactor," in Proc. Conf. and Workshop on Fusion Reanor Design, Madison, WJ, 1977 (IAEA, in preparation); also, Lawrence Livermore Laboratory, Rept. UCRL-80076 (1977).

20. G. A. Carlson, W. C. Condit, R. S. Devoto, J. H. Fink, J. D. Hanson, W. S. Neef, and A. C. Smith, Jr., Conceptual Design of the Field-Reversed Mirror Reactor, Lawrence Livermore Laboratory, Rept. UCRL52467 (in preparation).

21. W. C. Condit, G. A. Carlson, R. S. Devoto, J. N. Doggett, W. S. Neef, and J. D. Hanson, Preliminary' Design Calculations for a Field-Reversed Mirror Reacior, Law1ence Livermore Laboratory, Rept. UCR L-52170 (1976).

22. H. H. Fleischmann and T. Kammash, "System Analysis of the lon-Ring-Compressor Approach to Fusion," Nucl. Fusion 15, 1143 (1975).

23. R. H. Cohen, Tandem Mirror Rate Code and Cyclic Purging of Alphas in Tanden Reactors, Lawrence Livermore Laboratory, Rept. UCID-17544 (1977).

24. R. H. Cohen, M. E. Rensink, T. A. Cutler, and A. A. Mirin, "Collisional Loss of Electrostatically Confined Species in a Magnetic Mirror," Nucl. Fusion (in press).

25. M. N. Rosenbluth, "Superadiabaticity in Mirror Machines," Phys. Rev. Lett. 29, 408 (\$72). 
26. A. V. Timofeev, "Confinement of Charged Particles in Adiabatic Traps in the Presence of Monochromatic Cyclotron Oscillations," Nucl. Fusion 14, 165 (1974).

27. W. C. Turner, E. J. Powers, and T. C. Simonen, "Properties of Electrostatic Ion-Cyclotron Waves in a Mirror Machine," Phys. Rev. Lett. 39, 1087 (1977).

28. S. C. Luckhardt and H. H. Fleischmann, "Anomalous Fast-Particle Losses from Strong Electron Rings in Quadrupole-Stabilized Mirror Fields," Phys. Rev. Lett. 39, 747 (1977).

29. J. A. Byers, "Computer Simulation of the Ion-Ring-Compressor Approach to Fusion," Phys. Rev. Lett. 39, $1476(1977)$.

30. L. Spitzer, Jr., Physics of Fully Ionized Gases (Interscience, New York, 1962), p. 128.

31. H. L. Berk. Y. Matsuda, and J. J. Stewart, Lawrence Livermore Laboratory, private communication (1978).

32. A. K. Nekrasov, "Nonlinear Evolution of Drift-Cyclotron Flute Oscillations," Nud. Fusion 14, 865 (1975).

33. R E. Aamodt, Y. C. Lee, C. S. Liu, and M. N. Rosenbluth, "Nonlinear Dynamics of Drift-Cyclotron Instability," Phys. Rev. Lett. 39, 1660 (1977).

34. R. E. Aamodt and S. E. Bodner, "Nonlinear Dynamics of a Single Harmo nic Loss-Cone Flute Mode," Phys. Fluids 12, 1471 (1969). 